\title{
Alcohol Etherification via Alkoxy Radicals Generated by Visible-Light Photoredox Catalysis.
}

\author{
Alexandra R. Rivero, ${ }^{\dagger}$ Peter Fodran, ${ }^{\dagger}$ Alica Ondrejková $\dagger$ and Carl-Johan Wallentin* ${ }^{* \dagger}$ \\ $\dagger$ Department of Chemistry and Molecular Biology, University of \\ Gothenburg, Kemigården 4, 412 96, Gothenburg, Sweden. \\ e-mail : carl.wallentin@chem.gu.se
}

\section{TABLE OF CONTENTS}

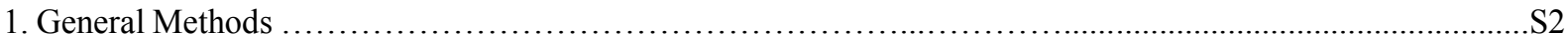

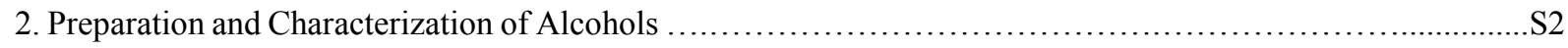

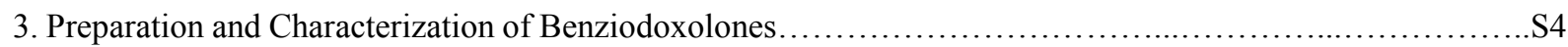

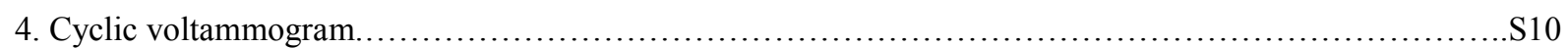

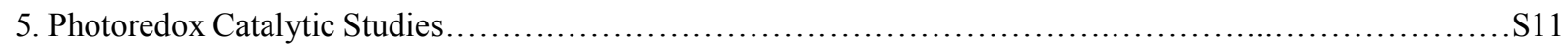

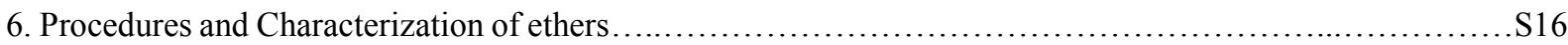

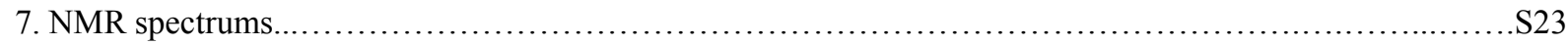




\section{GENERAL METHODS}

Unless otherwise indicated, chemicals were purchased from Sigma-Aldrich, Fluorochem and VWR Chemicals and were used without further purification. Air sensitive reactions were performed using oven-dried glassware (Toven $=150{ }^{\circ} \mathrm{C}$ ) and performed under a nitrogen atmosphere using Schlenk techniques. Solvents were dried on a solvent purification system (PS-MD-5/7 Inert technology). Reactions were monitored by thin-layer chromatography (TLC) on silica-gel-coated aluminum foils (silica gel 60 F254, Merck). The TLC plates were visualized by UV light $(\lambda=254 \mathrm{~nm})$ or by staining with the Seebach stain (phosphomolybdic acid $(2.5 \mathrm{~g}), \mathrm{Ce}\left(\mathrm{SO}_{4}\right){ }_{2}(1 \mathrm{~g}), \mathrm{CH}_{2} \mathrm{SO}_{4}$ $(6 \mathrm{ml})$, water $(94 \mathrm{ml}))$ and heating. Flash-column chromatography was performed on silica gel (VWR Chemicals, 40-63 $\mu \mathrm{m})$. NMR spectra were recorded on a Varian NMR 400 spectrometer at $25^{\circ} \mathrm{C}$. Chemical shifts $(\delta)$ are reported in ppm relative to the residual solvent peak. Splitting patterns are indicated as (s) singlet, (d) doublet, (t) triplet, (q) quartet, (m) multiplet and (br) broad. Coupling constants (J) are reported in Hertz (Hz). High-resolution mass spectra (HRMS) were recorded on a Waters XEVO-G2 QTOF mass spectrometer, using chemical ionization (CI) or electrospray ionization (ESI) in positive or negative mode, depending on the analyte. Melting points were determined on a Büchi melting point B-545 apparatus.

Photoredox reactions were performed using two Kessil lamps (A160WE). To avoid the heating produced by the lamps, two fans were employed to blow air through the reaction set-up, maintaining the reaction temperature around $30^{\circ} \mathrm{C}$ (see photos below).
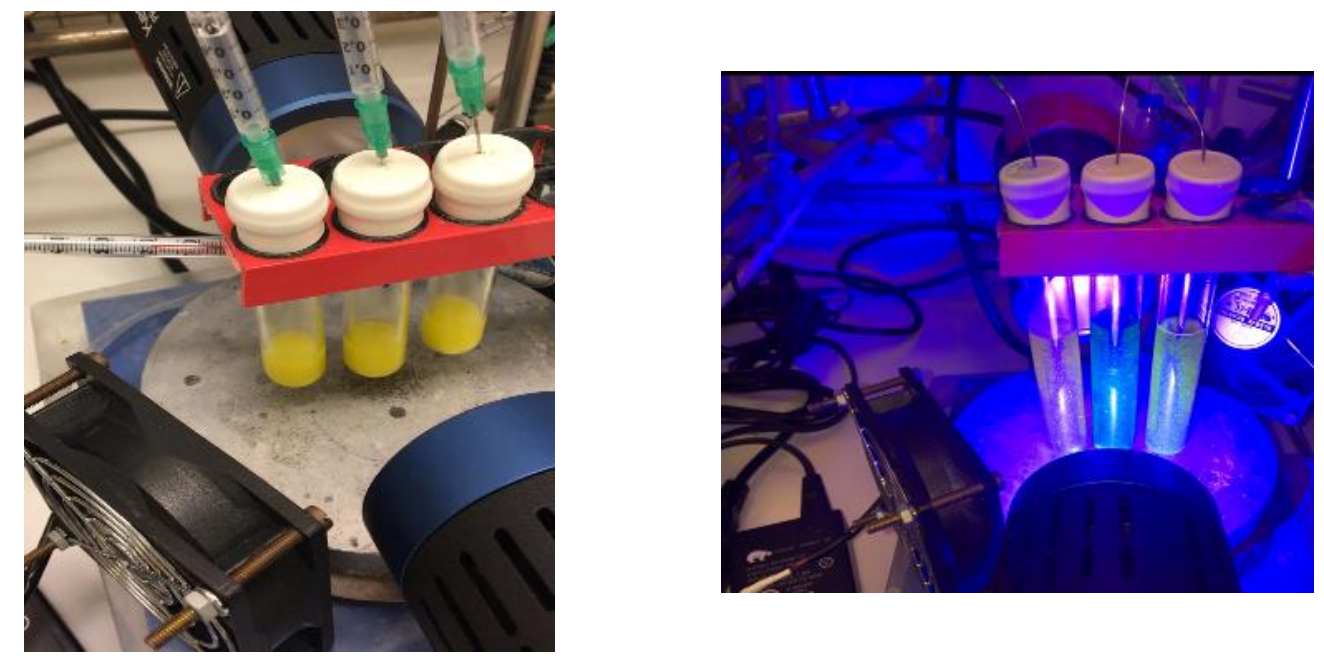

\section{PREPARATION AND CHARACTERIZATION OF ALCOHOLS}

When possible, alcohols were purchased from its commercially available source and were used without further

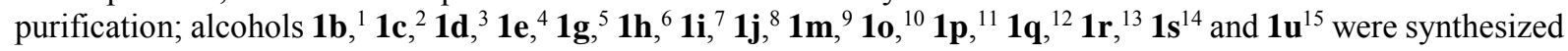
according to literature procedures and alcohols, $\mathbf{1 k}$ and $\mathbf{1 t}$ were synthesized as follows.

\footnotetext{
${ }^{1}$ Brand, J. P.; Charpentier, J.; Waser, J. Angew. Chem. Int. Ed. 2009, 48, 9346.

${ }^{2}$ Dammalapati, V. L. N. R.; Mudduluru, H. K.; Aduri, R. Process for the preparation of vilanterol and intermediates thereof. U.S. Patent $20150239862 \mathrm{~A} 1$, August 27, 2015.

${ }^{3}$ Lewis, F. D.; Wu, Y.; Liu, X. J. Am. Chem. Soc. 2002, 124, 12165

${ }^{4}$ Li, K.; Ran, L.; Yu, Y-H.; Tang, Y. J. Org. Chem. 2004, 69, 3986.

${ }^{5}$ Tang, J.; Maddali, K.; Metifiot, M.; Sham, Y. Y.; Vince, R.; Pommier, Y.; Wang, Z. J. Med. Chem. 2011, $54,2282$.

${ }^{6}$ Wong, W. C.; Sun, W.; Cui, W.; Chen, Y.; Forray, C.; Vaysse, P. J.-J.; Branchek, T. A.; Gluchowski, C. J. Med. Chem. $2000,43,1699$.

${ }^{7}$ Zwierzak A.; Pines H. J. Org. Chem.1963, 28, 12, 3392.

${ }^{8}$ Kropp, P. J.; Breton, G. W.; Craig, S. L.; Crawford, S. D.; Durland, Jr., W. F.; Jones III, J. E; Raleigh, J. S. J. Org. Chem. 1995, 60, 4146.

${ }^{9}$ McMills, M. C.; Wright, D. L.; Weekly, R. M. Synth. Commun. 2002, 32, 2417.

${ }^{10}$ Taylor, E. C.; Gillespie, P. J. Org. Chem. 1992, 57, 5757.

${ }^{11}$ Hazelden, I. R.; Carmona, R. C.; Langer, T.; Pringle, P. G.; Bower, J. F. Angew. Chem. Int. Ed. 2018, $57,5124$.

${ }^{12}$ Stauffer, S. R.; Hartwig, J. F. J. Am. Chem. Soc. 2003 125, 6977.

${ }^{13}$ Kim, J. K.; Kim, H. D.; Seo, M. J.; Kim, H. R.; No, Z.; Hab, D.; Leea, G. H. Tetrahedron Lett. 2006, 47, 9.

${ }^{14}$ Anchel; M.; Blatt, A. H. J. Am. Chem. Soc. 1941, 63, 1948.

${ }^{15}$ Weinhouse, K. J. Org. Chem. 1936, 1, 209.
} 


\subsection{General method A for the preparation of alcohols $1 \mathrm{k}$ and $1 \mathrm{t}$}

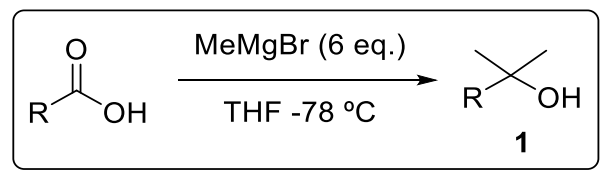

To a solution of the corresponding acid (1 eq.) in anhydrous THF $(1 \mathrm{~mL} / \mathrm{mmol})$ was added $\mathrm{MeMgBr}(6$ eq. $)$ dropwise at $-78^{\circ} \mathrm{C}$. The reaction was then allowed to warm up to room temperature and refluxed for $4 \mathrm{~h}$. After completion, the reaction was quenched with $1 \mathrm{~N}$ aqueous $\mathrm{NH}_{4} \mathrm{Cl}$ at $0{ }^{\circ} \mathrm{C}$ and the organic materials were extracted twice with diethyl ether. The combined extracts were washed with brine, and dried over MgSO4. Volatile materials were removed in vacuo and the crude material was purified by flash column chromatography (silica gel, pentane/ether: 80/20) to give the corresponding alcohol as a colourless oil.

\section{2-(2-benzylphenyl)propan-2-ol, alcohol $\mathbf{1} \mathbf{k}^{16}$}<smiles>CC(C)(O)c1ccccc1Cc1ccccc1</smiles>

Following general method A, alcohol $\mathbf{1 k}$ was obtained as collorless oil in $61 \%$ yield $(6.1 \mathrm{mmol}, 1.4 \mathrm{~g})$.

${ }^{1} \mathbf{H}$ NMR (400 MHz, CDCl $)$ ): $\delta 7.51-7.42(\mathrm{~m}, 1 \mathrm{H}), 7.36-7.07(\mathrm{~m}, 8 \mathrm{H}), 4.46(\mathrm{~s}, 2 \mathrm{H}), 1.66(\mathrm{~s}, 6 \mathrm{H})$.

${ }^{13} \mathbf{C}$ NMR (101 MHz, $\left.\mathbf{C D C l}_{3}\right): \delta 146.1,142.8,138.5,133.4,128.9,128.5,128.5,127.3,126.2,125.8,125.8,74.1$, $39.9,32.0$.

\section{2-methyl-4-(naphthalen-2-yl)butan-2-ol, alcohol $\mathbf{1 t}^{17}$}<smiles>CC(C)(O)CCc1cccc2ccccc12</smiles>

Following general method $\mathrm{A}$, alcohol $\mathbf{1 t}$ was obtained as collorless oil in $78 \%$ yield $(7.8 \mathrm{mmol}, 1.7 \mathrm{~g})$.

${ }^{1} \mathbf{H}$ NMR $\left(400 \mathrm{MHz}, \mathbf{C D C l}_{3}\right): \delta 8.09-8.04(\mathrm{~m}, 1 \mathrm{H}), 7.89-7.83(\mathrm{~m}, 1 \mathrm{H}), 7.74-7.69(\mathrm{~m}, 1 \mathrm{H}), 7.55-7.45(\mathrm{~m}$, $2 \mathrm{H}), 7.43-7.33(\mathrm{~m}, 2 \mathrm{H}), 3.30-3.10(\mathrm{~m}, 2 \mathrm{H}), 1.96-1.83(\mathrm{~m}, 2 \mathrm{H}), 1.38(\mathrm{~s}, 6 \mathrm{H})$.

${ }^{13}$ C NMR (101 MHz, CDCl $): \delta 138.8,134.0,131.9,128.8,126.6,125.9,125.8,125.7,125.5,123.8,71.0,45.0$, 29.4, 27.9.

HRMS (ESI): Exact mass calculated for $[\mathrm{M}+\mathrm{H}]^{+}\left(-\mathrm{H}_{2} \mathrm{O}\right)\left(\mathrm{C}_{15} \mathrm{H}_{19} \mathrm{O}\right) \mathrm{m} / \mathrm{z}$ 197.1330, found 197.1250.

\footnotetext{
${ }^{16}$ Falshaw, C. P.; Hashi, N. A.; Taylor, G. A. J. Chem. Soc. Perkin Trans 1985, 1, 1837.

${ }^{17}$ Qihang, G.; Xiang, R.; Zhan, L. Org. Lett. 2019, 21, 880.
} 


\section{PREPARATION AND CHARACTERIZATION OF BENZIODOXOLONES}

\subsection{General method B for the preparation of benziodoxolones}

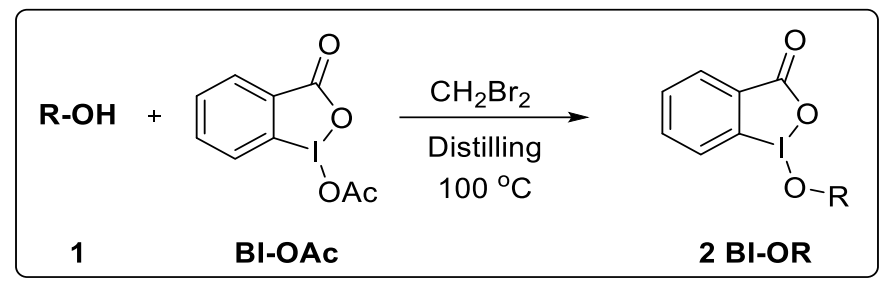

The corresponding alcohol (4.9 mmol, 1.5 eq.) and 1-acetoxy-1,2-benziodoxol-3(1H)-one (BI-OAc) ${ }^{18}(1 \mathrm{~g}, 3.3$ mmol, 1 eq.) were added to a $10 \mathrm{~mL}$ round bottomed flask provided with a stirring bar and a distillation system, followed by $10 \mathrm{~mL}$ of dry dibromomethane. The resulting solution was heated to $100{ }^{\circ} \mathrm{C}$ allowing the azeotrope of dibromomethane and acetic acid distil; the heat was maintained until all solvent was distilled. The resulting white gum was suspended in $5 \mathrm{~mL}$ of ether and allowed to crystallised in the freezer for $24 \mathrm{~h}$. After that period, the resulting white powder was filtered and washed with cold ether to afford the corresponding benziodoxolones.

The choice of solvent was critical as utilization of $\mathrm{CH}_{2} \mathrm{Br}_{2}$ leads to complete solubilisation of the reagents. Furthermore, $\mathrm{CH}_{2} \mathrm{Br}_{2}$ forms a rich azeotrope with formed acetic acid thus shifting the equilibrium towards the products.

Note 1: The process can also be performed at room temperature by distilling off the azeotrope under reduce pressure without significant yield drop.

Note 2: Larger amounts of alcohols do not increase the reaction yields and difficulties the crystallization process, smaller amounts of alcohols result in side products that precipitates together with the benziodoxolones.

Note 3: For tertiary alcohols, the distillation process is repeated three times.

\section{Benziodoxolone 2a}<smiles>[B]OCCOCc1ccccc1</smiles>

Following general method $\mathrm{B}$, benziodoxolone 2a was obtained as white powder in $80 \%$ yield.

${ }^{1} \mathbf{H}$ NMR (400 MHz, $\left.\mathbf{C D C l}_{3}\right): \delta 8.23(\mathrm{dd}, J=7.5,1.6 \mathrm{~Hz}, 1 \mathrm{H}), 7.92(\mathrm{dd}, J=8.3,1.0 \mathrm{~Hz}, 1 \mathrm{H}), 7.80$ (ddd, $J=8.3$, $7.2,1.6 \mathrm{~Hz}, 1 \mathrm{H}), 7.63(\mathrm{td}, J=7.5,1.0 \mathrm{~Hz}, 1 \mathrm{H}), 7.46-7.26(\mathrm{~m}, 5 \mathrm{H}), 4.65(\mathrm{~s}, 2 \mathrm{H}), 4.44-4.40$ (m, 2H), $3.71-3.67$ $(\mathrm{m}, 2 \mathrm{H})$.

${ }^{13} \mathbf{C}$ NMR (101 MHz, $\left.\mathbf{C D C l}_{3}\right) ; \delta 168.6,137.3,134.8,132.6,130.6,130.4,128.7,128.2,127.9,126.6,120.0,73.7$, 72.2, 71.9 .

HRMS (ESI): Exact mass calculated for $[\mathrm{M}+\mathrm{H}]^{+}\left(\mathrm{C}_{16} \mathrm{H}_{16} \mathrm{IO}_{4}\right) \mathrm{m} / \mathrm{z} 399.0093$, found 399.0088 .

MP: $101-103^{\circ} \mathrm{C}$.

Benziodoxolone 2b<smiles>COc1ccc(COCC[18F])cc1</smiles>

Following general method B, benziodoxolone $\mathbf{2 b}$ was obtained as white powder in $73 \%$ yield.

${ }^{1} \mathbf{H}$ NMR (400 MHz, $\left.\mathbf{C D C l}_{3}\right): \delta 8.23(\mathrm{dd}, J=7.6,1.5 \mathrm{~Hz}, 1 \mathrm{H}), 7.93-7.89(\mathrm{~m}, 1 \mathrm{H}), 7.81$ (ddd, $J=8.3,7.2,1.5$ $\mathrm{Hz}, 1 \mathrm{H}), 7.64(\mathrm{td}, J=7.4,1.0 \mathrm{~Hz}, 1 \mathrm{H}), 7.30(\mathrm{~d}, J=8.7 \mathrm{~Hz}, 2 \mathrm{H}), 6.92(\mathrm{~d}, J=8.7 \mathrm{~Hz}, 2 \mathrm{H}), 4.58(\mathrm{~s}, 2 \mathrm{H}), 4.44-4.37$ (m, 2H), $3.83(\mathrm{~s}, 3 \mathrm{H}), 3.70-3.63(\mathrm{~m}, 1 \mathrm{H})$.

${ }^{13}$ C NMR (101 MHz, CDCl $): \delta 168.7,159.7,134.9,132.7,130.7,130.6,129.7,129.5,126.8,120.2,114.2,73.5$, $72.3,71.7,55.4$.

\footnotetext{
${ }^{18}$ Hendrick, C. E.; Bitting, K. J.; Cho, S.; Wang, Q. J. Am. Chem. Soc. 2017, 139, 11622.
} 
HRMS (ESI): Exact mass calculated for $[\mathrm{M}+\mathrm{H}]^{+}\left(\mathrm{C}_{17} \mathrm{H}_{18} \mathrm{IO}_{5}\right) \mathrm{m} / \mathrm{z} 429.0193$, found 429.0193 . MP: $103-105{ }^{\circ} \mathrm{C}$.

$\underline{\text { Benziodoxolone 2c }}$<smiles>FCOCCOCc1c(Cl)cccc1Cl</smiles>

Following general method B, benziodoxolone $2 \mathrm{c}$ was obtained as white powder in $97 \%$ yield.

${ }^{1}$ H NMR (400 MHz, CDCl $)$ : $\delta 8.19(\mathrm{dd}, J=7.6,1.5 \mathrm{~Hz}, 0 \mathrm{H}), 7.89-7.85(\mathrm{~m}, 0 \mathrm{H}), 7.82-7.71(\mathrm{~m}, 0 \mathrm{H}), 7.60(\mathrm{td}$, $J=7.4,1.0 \mathrm{~Hz}, 0 \mathrm{H}), 7.39-7.34(\mathrm{~m}, 1 \mathrm{H}), 7.31-7.19(\mathrm{~m}, 1 \mathrm{H}), 4.91(\mathrm{~s}, 1 \mathrm{H}), 4.60-4.15(\mathrm{~m}, 1 \mathrm{H}), 3.79-3.75(\mathrm{~m}$, $1 \mathrm{H})$.

${ }^{13}$ C NMR (101 MHz, CDCl $): \delta 168.8,136.7,134.8,132.6,130.6,130.6,130.5,128.8,126.7,120.2,72.7,72.1$, 67.8 .

HRMS (ESI): Exact mass calculated for $[\mathrm{M}+\mathrm{H}]^{+}\left(\mathrm{C}_{16} \mathrm{H}_{14} \mathrm{Cl}_{2} \mathrm{IO}_{4}\right) \mathrm{m} / \mathrm{z} 466.9316$, found 466.9308 .

MP: $126-128^{\circ} \mathrm{C}$

$\underline{\text { Benziodoxolone 2d }}$

$\mathbf{B I}$<smiles>COCCCCOc1ccccc1</smiles>

Following general method B, benziodoxolone 2d was obtained as white powder in $74 \%$ yield.

${ }^{1}$ H NMR (400 MHz, CDCl $): \delta 8.21(\mathrm{dd}, J=7.6,1.5 \mathrm{~Hz}, 1 \mathrm{H}), 7.86-7.77(\mathrm{~m}, 1 \mathrm{H}), 7.70(\mathrm{dd}, J=8.1,0.8 \mathrm{~Hz}, 1 \mathrm{H})$, $7.62(\mathrm{td}, J=7.6,0.8 \mathrm{~Hz}, 1 \mathrm{H}), 7.25-7.19(\mathrm{~m}, 2 \mathrm{H}), 6.92-6.86(\mathrm{~m}, 1 \mathrm{H}), 6.83(\mathrm{dd}, J=8.8,1.0 \mathrm{~Hz}, 2 \mathrm{H}), 4.24(\mathrm{t}, J=$ $6.1 \mathrm{~Hz}, 2 \mathrm{H}), 3.97(\mathrm{t}, J=5.8 \mathrm{~Hz}, 2 \mathrm{H}), 1.99-1.73(\mathrm{~m}, 4 \mathrm{H})$.

${ }^{13}$ C NMR (101 MHz, CDCl 3$): ~ \delta{ }^{13} \mathrm{C}$ NMR $\left(101 \mathrm{MHz}, \mathrm{cdcl}_{3}\right) \delta 168.1,158.9,135.2,133.0,131.1,130.8,129.6$, 126.0, 120.9, 119.0, 114.6, 74.0, 67.4, 30.2, 25.8 .

HRMS (ESI): Exact mass calculated for $[\mathrm{M}+\mathrm{H}]^{+}\left(\mathrm{C}_{17} \mathrm{H}_{18} \mathrm{IO}_{4}\right) \mathrm{m} / \mathrm{z} 413.0244$, found 413.0244 .

MP. $117-119^{\circ} \mathrm{C}$.

Benziodoxolone 2e<smiles>[Bi]OCCOC1CCCCO1</smiles>

Following general method B, benziodoxolone $2 \mathrm{e}$ was obtained as white powder in $90 \%$ yield.

${ }^{1} \mathbf{H}$ NMR (400 MHz, CDCl 3$): \delta 8.25(\mathrm{dd}, J=7.6,1.4 \mathrm{~Hz}, 1 \mathrm{H}), 7.99-7.92(\mathrm{~m}, 1 \mathrm{H}), 7.90-7.83(\mathrm{~m}, 1 \mathrm{H}), 7.71-$ $7.60(\mathrm{~m}, 1 \mathrm{H}), 4.70(\mathrm{dd}, J=4.5,2.6 \mathrm{~Hz}, 1 \mathrm{H}), 4.52-4.38(\mathrm{~m}, 2 \mathrm{H}), 3.96(\mathrm{ddd}, J=11.0,6.2,3.0 \mathrm{~Hz}, 1 \mathrm{H}), 3.90$ (ddd, $J=11.0,7.8,3.3 \mathrm{~Hz}, 1 \mathrm{H}), 3.63(\mathrm{ddd}, J=10.8,6.2,3.3 \mathrm{~Hz}, 1 \mathrm{H}), 3.60-3.51(\mathrm{~m}, 1 \mathrm{H}), 1.99-1.76(\mathrm{~m}, 2 \mathrm{H}), 1.76-$ $1.48(\mathrm{~m}, 4 \mathrm{H})$.

${ }^{13}$ C NMR (101 MHz, CDCl 3$): \delta 168.7,134.9,132.8,130.8,130.6,126.8,120.1,99.6,72.5,69.5,62.8,31.0,25.4$, 19.9.

HRMS (ESI): Exact mass calculated for $[\mathrm{M}+\mathrm{H}]^{+}\left(\mathrm{C}_{14} \mathrm{H}_{18} \mathrm{IO}_{5}\right) \mathrm{m} / \mathrm{z} 393.0197$, found 393.0193 .

MP: $89-91{ }^{\circ} \mathrm{C}$.

\section{Benziodoxolone 2f}

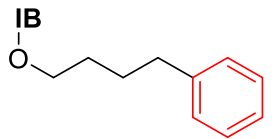

Following general method B, benziodoxolone $2 \mathbf{f}$ was obtained as white powder in $74 \%$ yield.

${ }^{1}$ H NMR (400 MHz, CDCl3): $\delta 8.28(\mathrm{dd}, J=7.6,1.6 \mathrm{~Hz}, 1 \mathrm{H}), 7.87(\mathrm{ddd}, J=8.6,7.2,1.6 \mathrm{~Hz}, 1 \mathrm{H}), 7.77-7.71$ $(\mathrm{m}, 1 \mathrm{H}), 7.71-7.65(\mathrm{~m}, 1 \mathrm{H}), 7.34-7.27(\mathrm{~m}, 2 \mathrm{H}), 7.24-7.16(\mathrm{~m}, 3 \mathrm{H}), 4.22(\mathrm{t}, J=6.2 \mathrm{~Hz}, 2 \mathrm{H}), 2.69(\mathrm{t}, J=7.1$ $\mathrm{Hz}, 2 \mathrm{H}), 1.86-1.65(\mathrm{~m}, 4 \mathrm{H})$.

${ }^{13}$ C NMR (101 MHz, CDCl $)$ : $\delta$ 168.0, 142.0, 135.1, 133.0, 131.1, 130.8, 128.6, 128.6, 126.2, 126.0, 119.0, 74.3, 35.7, 33.0, 27.5.

HRMS (ESI): Exact mass calculated for $[\mathrm{M}+\mathrm{H}]^{+}\left(\mathrm{C}_{17} \mathrm{H}_{18} \mathrm{IO}_{3}\right) \mathrm{m} / \mathrm{z} 397.0287$, found 397.0295 . 
MP: $57-89^{\circ} \mathrm{C}$.

Benziodoxolone 2g<smiles>Fc1ccc(CCCCOC(F)F)cc1</smiles>

Following general method $\mathrm{B}$, benziodoxolone $\mathbf{2 g}$ was obtained as white powder in $80 \%$ yield.

${ }^{1}$ H NMR (400 MHz, CDCl $)$ ): $\delta 8.28$ (ddd, $\left.J=7.5,1.6,0.4 \mathrm{~Hz}, 1 \mathrm{H}\right), 7.87$ (ddd, $\left.J=8.3,7.2,1.6 \mathrm{~Hz}, 1 \mathrm{H}\right), 7.73$ (ddd, $J=8.3,1.0,0.4 \mathrm{~Hz}, 1 \mathrm{H}), 7.74-7.65(\mathrm{~m}, 1 \mathrm{H}), 7.19-7.06(\mathrm{~m}, 2 \mathrm{H}), 7.03-6.85(\mathrm{~m}, 2 \mathrm{H}), 4.23(\mathrm{t}, J=6.2 \mathrm{~Hz}$, $2 \mathrm{H}), 2.65$ (t, $J=7.0 \mathrm{~Hz}, 2 \mathrm{H}), 2.01-1.34(\mathrm{~m}, 4 \mathrm{H})$.

${ }^{13}$ C NMR (101 MHz, CDCl $): \delta 168.1,161.3(\mathrm{~d}, J=243.4 \mathrm{~Hz}), 137.6(\mathrm{~d}, J=3.2 \mathrm{~Hz}), 135.1,132.8,131.0,130.8$, $129.8(\mathrm{~d}, J=7.8 \mathrm{~Hz}), 126.0,119.0,115.2(\mathrm{~d}, J=21.1 \mathrm{~Hz}), 74.2,34.8,32.8,27.7$.

HRMS (ESI): Exact mass calculated for $[\mathrm{M}+\mathrm{H}]^{+}\left(\mathrm{C}_{17} \mathrm{H}_{17} \mathrm{FIO}_{3}\right) \mathrm{m} / \mathrm{z} 415.0199$, found 415.0201 .

MP: $91-93{ }^{\circ} \mathrm{C}$.

$\underline{\text { Benziodoxolone 2h }}$

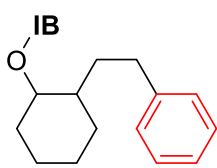

Following general method B, benziodoxolone $\mathbf{2 h}$ was obtained as white powder in $73 \%$ yield.

${ }^{1}$ H NMR (400 MHz, CDCl 3$): \delta 8.23(\mathrm{dd}, J=7.6,1.4 \mathrm{~Hz}, 1 \mathrm{H}), 7.85(\mathrm{dd}, J=8.2,1.1 \mathrm{~Hz}, 1 \mathrm{H}), 7.78(\mathrm{ddd}, J=8.4$, $7.1,1.6 \mathrm{~Hz}, 1 \mathrm{H}), 7.64(\mathrm{td}, J=7.4,1.1 \mathrm{~Hz}, 1 \mathrm{H}), 7.35-7.15(\mathrm{~m}, 5 \mathrm{H}), 3.61(\mathrm{td}, J=9.6,4.0 \mathrm{~Hz}, 1 \mathrm{H}), 2.79(\mathrm{ddd}, J=$ $14.6,9.5,5.5 \mathrm{~Hz}, 1 \mathrm{H}), 2.60(\mathrm{ddd}, J=13.8,9.0,6.9 \mathrm{~Hz}, 1 \mathrm{H}), 2.16(\mathrm{dddd}, J=12.7,9.7,6.9,3.1 \mathrm{~Hz}, 1 \mathrm{H}), 2.04-$ $1.94(\mathrm{~m}, 2 \mathrm{H}), 1.83-1.04(\mathrm{~m}, 7 \mathrm{H})$.

${ }^{13}$ C NMR (101 MHz, CDCl3): $\delta$ 168.2, 142.7, 134.8, 132.7, 130.9, 130.8, 128.7, 128.6, 126.6, 126.2, 120.1, 85.2, 44.9, 35.2, 34.1, 33.0, 30.4, 25.2, 24.9 .

HRMS (ESI): Exact mass calculated for $[\mathrm{M}+\mathrm{H}]^{+}\left(\mathrm{C}_{21} \mathrm{H}_{24} \mathrm{IO}_{3}\right) \mathrm{m} / \mathrm{z} 451.0773$, found 451.0765 .

MP: $72-76^{\circ} \mathrm{C}$.

$\underline{\text { Benziodoxolone 2i }}$<smiles>[PH3-]C(CCCc1ccccc1)c1ccccc1</smiles>

Following general method $\mathrm{B}$, benziodoxolone $2 \mathbf{i}$ was obtained as white powder in $67 \%$ yield.

${ }^{1}$ H NMR (400 MHz, CDCl $): \delta 8.24(\mathrm{ddd}, J=7.5,1.5,0.5 \mathrm{~Hz}, 1 \mathrm{H}), 7.84-7.74(\mathrm{~m}, 2 \mathrm{H}), 7.65(\mathrm{ddd}, J=7.5,6.5$, $1.7 \mathrm{~Hz}, 1 \mathrm{H}), 7.43-7.25(\mathrm{~m}, 7 \mathrm{H}), 7.23-7.12(\mathrm{~m}, 3 \mathrm{H}), 4.88(\mathrm{t}, J=6.6 \mathrm{~Hz}, 1 \mathrm{H}), 2.75-2.56(\mathrm{~m}, 2 \mathrm{H}), 2.14-2.00$ $(\mathrm{m}, 1 \mathrm{H}), 1.97-1.69(\mathrm{~m}, 2 \mathrm{H}), 1.71-1.49(\mathrm{~m}, 1 \mathrm{H})$.

${ }^{13}$ C NMR (101 MHz, CDCl3): $\delta$ 168.0, 143.3, 141.8, 135.1, 132.9, 131.0, 130.7, 129.3, 129.0, 128.5, 128.5, 126.7, 126.4, 126.1, 119.1, 85.0, 39.2, 35.8, 27.7.

HRMS (ESI): Exact mass calculated for $[\mathrm{M}+\mathrm{H}]^{+}\left(\mathrm{C}_{23} \mathrm{H}_{22} \mathrm{IO}_{3}\right) \mathrm{m} / \mathrm{z} 473.0602$, found 473.0608 .

MP: $79-82^{\circ} \mathrm{C}$.

\section{Benziodoxolone 2j}<smiles>C[B]OC(C)(C)CCCc1ccccc1</smiles>

Following general method B, benziodoxolone $\mathbf{2} \mathbf{j}$ was obtained as white powder in $79 \%$ yield.

${ }^{1} \mathbf{H}$ NMR (400 MHz, CDCl 3$): \delta 8.29-8.21(\mathrm{~m}, 1 \mathrm{H}), 7.85-7.80(\mathrm{~m}, 2 \mathrm{H}), 7.68-7.60$ (m, 1H), $7.35-7.26(\mathrm{~m}$, 2H), $7.28-7.15(\mathrm{~m}, 3 \mathrm{H}), 2.66(\mathrm{t}, J=7.2 \mathrm{~Hz}, 2 \mathrm{H}), 1.86-1.69(\mathrm{~m}, 2 \mathrm{H}), 1.72-1.54(\mathrm{~m}, 2 \mathrm{H}), 1.31(\mathrm{~s}, 6 \mathrm{H})$. 
${ }^{13}$ C NMR (101 MHz, CDCl3): $\delta$ 168.2, 142.0, 134.7, 132.6, 131.1, 130.8, 128.7, 128.7, 126.5, 126.4, 119.8, 80.4, 42.7, 36.0, 28.4, 26.5.

HRMS (ESI): Exact mass calculated for $[\mathrm{M}+\mathrm{H}]^{+}\left(\mathrm{C}_{19} \mathrm{H}_{22} \mathrm{IO}_{3}\right) \mathrm{m} / \mathrm{z} 425.0599$, found 425.0608 .

MP: $96-98^{\circ} \mathrm{C}$.

$\underline{\text { Benziodoxolone 2k }}$

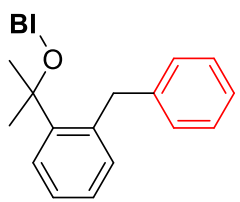

Following general method B, benziodoxolone $2 \mathbf{k}$ was obtained as white powder in $46 \%$ yield.

${ }^{1} \mathbf{H}$ NMR (400 MHz, $\left.\mathbf{C D C l}_{3}\right): \delta 8.21(\mathrm{ddd}, J=7.5,1.6,0.4 \mathrm{~Hz}, 1 \mathrm{H}), 7.97(\mathrm{ddd}, J=8.3,1.0,0.4 \mathrm{~Hz}, 1 \mathrm{H}), 7.84$ $(\mathrm{ddd}, J=8.3,7.2,1.6 \mathrm{~Hz}, 1 \mathrm{H}), 7.64(\mathrm{td}, J=7.4,1.0 \mathrm{~Hz}, 1 \mathrm{H}), 7.42(\mathrm{dd}, J=7.5,1.3 \mathrm{~Hz}, 1 \mathrm{H}), 7.40-7.30(\mathrm{~m}, 1 \mathrm{H})$, $7.32-7.22(\mathrm{~m}, 4 \mathrm{H}), 7.23-7.14(\mathrm{~m}, 1 \mathrm{H}), 7.15-7.07(\mathrm{~m}, 2 \mathrm{H}), 4.56(\mathrm{~s}, 2 \mathrm{H}), 1.81(\mathrm{~s}, 6 \mathrm{H})$.

${ }^{13}$ C NMR (101 MHz, CDCl $)$ : $\delta$ 168.1, 144.2, 142.0, 138.9, 134.8, 134.6, 132.6, 131.0, 130.8, 128.9, 128.9, 128.7, $127.1,127.0,126.5,126.5,120.0,82.0,39.4,30.4$.

HRMS (ESI): Exact mass calculated for $[\mathrm{M}+\mathrm{H}]^{+}\left(\mathrm{C}_{23} \mathrm{H}_{22} \mathrm{IO}_{3}\right) \mathrm{m} / \mathrm{z} 473.0620$, found 473.0608 .

MP: $134-137^{\circ} \mathrm{C}$.

$\underline{\text { Benziodoxolone 2l }}$

$\mathrm{BI}$<smiles>[AlH2]OCCCc1ccccc1</smiles>

Following general method B, benziodoxolone $2 \mathbf{l}$ was obtained as white powder in $80 \%$ yield.

${ }^{1} \mathbf{H}$ NMR (400 MHz, CDCl 3 ): $\delta 8.26(\mathrm{dd}, J=7.5,1.6 \mathrm{~Hz}, 1 \mathrm{H}), 7.85(\mathrm{ddd}, J=8.5,7.2,1.6 \mathrm{~Hz}, 1 \mathrm{H}), 7.72(\mathrm{dd}, J=$ 8.5, 1.0 Hz, 1H), $7.67(\mathrm{td}, J=7.5,1.0 \mathrm{~Hz}, 1 \mathrm{H}), 7.35-7.29(\mathrm{~m}, 2 \mathrm{H}), 7.25-7.19(\mathrm{~m}, 3 \mathrm{H}), 4.24(\mathrm{t}, J=6.4 \mathrm{~Hz}, 2 \mathrm{H})$, $2.78(\mathrm{t}, J=7.6 \mathrm{~Hz}, 2 \mathrm{H}), 2.06-1.99(\mathrm{~m}, 2 \mathrm{H})$.

${ }^{13}$ C NMR (101 MHz, CDCl3): $\delta$ 168.0, 141.2, 135.1, 132.9, 131.1, 130.7, 128.7, 128.6, 126.3, 126.0, 119.0, 73.6, 34.7, 32.0.

HRMS (ESI): Exact mass calculated for $[\mathrm{M}+\mathrm{H}]^{+}\left(\mathrm{C}_{16} \mathrm{H}_{16} \mathrm{IO}_{3}\right) \mathrm{m} / \mathrm{z} 383.0141$, found 383.0139.

MP: $95-97{ }^{\circ} \mathrm{C}$.

$\underline{\text { Benziodoxolone 2m }}$<smiles>COc1cc(CCCOBr)cc(OC)c1OC</smiles>

Following general method B, benziodoxolone $\mathbf{2 m}$ was obtained as white powder in $87 \%$ yield.

${ }^{1}$ H NMR (400 MHz, CDCl3): $\delta 8.26(\mathrm{dd}, J=7.6,1.5 \mathrm{~Hz}, 1 \mathrm{H}), 7.86(\mathrm{ddd}, J=8.2,7.2,1.5 \mathrm{~Hz}, 1 \mathrm{H}), 7.76-7.68$ $(\mathrm{m}, 1 \mathrm{H}), 7.73-7.64(\mathrm{~m}, 1 \mathrm{H}), 6.43(\mathrm{~s}, 2 \mathrm{H}), 4.25(\mathrm{t}, J=6.4 \mathrm{~Hz}, 2 \mathrm{H}), 3.85(\mathrm{~s}, 6 \mathrm{H}), 3.83(\mathrm{~s}, 3 \mathrm{H}), 2.72(\mathrm{~d}, J=6.7$ $\mathrm{Hz}, 2 \mathrm{H}), 2.05-1.96(\mathrm{~m}, 2 \mathrm{H})$.

${ }^{13}$ C NMR (101 MHz, CDCl $): \delta 167.8,153.3,136.8,136.4,135.0,132.9,131.0,130.6,125.9,118.8,105.3$, 73.4, 60.9, 56.1, 34.7, 32.3 .

HRMS (ESI): Exact mass calculated for $[\mathrm{M}+\mathrm{H}]^{+}\left(\mathrm{C}_{19} \mathrm{H}_{22} \mathrm{IO}_{6}\right) \mathrm{m} / \mathrm{z} 473.0465$, found 473.0456 .

MP: $119-122^{\circ} \mathrm{C}$.

$\underline{\text { Benziodoxolone 2n }}$

BI<smiles>COc1ccc(CCCO[AlH2])cc1</smiles>

Following general method B, benziodoxolone 2n was obtained as white powder in $52 \%$ yield.

${ }^{1}$ H NMR (400 MHz, CDCl $\left.\mathbf{~}_{3}\right): \delta 8.27$ (ddd, J = 7.6, 1.6, $\left.0.5 \mathrm{~Hz}, 1 \mathrm{H}\right), 7.86$ (ddd, J = 8.3, 7.2, $1.6 \mathrm{~Hz}, 1 \mathrm{H}$ ), $7.75-$ $7.63(\mathrm{~m}, 2 \mathrm{H}), 7.13(\mathrm{~d}, \mathrm{~J}=8.8 \mathrm{~Hz}, 1 \mathrm{H}), 6.86(\mathrm{~d}, \mathrm{~J}=8.6 \mathrm{~Hz}, 2 \mathrm{H}), 4.22(\mathrm{t}, \mathrm{J}=6.4 \mathrm{~Hz}, 2 \mathrm{H}), 3.80(\mathrm{~s}, 3 \mathrm{H}), 2.72(\mathrm{dd}, \mathrm{J}$ $=8.0,7.0 \mathrm{~Hz}, 2 \mathrm{H}), 2.06-1.94(\mathrm{~m}, 2 \mathrm{H})$. 
${ }^{13}$ C NMR (101 MHz, CDCl3): $\delta$ 168.1, 158.2, 135.2, 133.1, 133.0, 131.1, 130.8, 129.5, 126.1, 119.0, 114.1, 77.2, 73.6, 55.4, 34.9, 31.1.

HRMS (ESI): Exact mass calculated for $[\mathrm{M}+\mathrm{H}]^{+}\left(\mathrm{C}_{17} \mathrm{H}_{18} \mathrm{IO}_{4}\right) \mathrm{m} / \mathrm{z} 413.0244$, found 413.0247 .

MP: $78-80^{\circ} \mathrm{C}$.

$\underline{\text { Benziodoxolone 2o }}$

Bl、<smiles>COC(=O)c1ccc(CCCOI)cc1</smiles>

Following general method B, benziodoxolone 20 was obtained as white powder in $56 \%$ yield.

${ }^{1} \mathbf{H}$ NMR (400 MHz, CDCl$): \delta 8.25(\mathrm{dd}, \mathrm{J}=7.6,1.5 \mathrm{~Hz}, 1 \mathrm{H}), 7.97(\mathrm{~d}, \mathrm{~J}=8.2 \mathrm{~Hz}, 2 \mathrm{H}), 7.85(\mathrm{ddd}, \mathrm{J}=8.1,7.2$, $1.6 \mathrm{~Hz}, 1 \mathrm{H}), 7.74-7.63(\mathrm{~m}, 2 \mathrm{H}), 7.28(\mathrm{~d}, \mathrm{~J}=8.2 \mathrm{~Hz}, 2 \mathrm{H}), 4.22(\mathrm{t}, \mathrm{J}=6.4 \mathrm{~Hz}, 2 \mathrm{H}), 3.90(\mathrm{~s}, 3 \mathrm{H}), 2.82(\mathrm{dd}, \mathrm{J}=$ $8.5,6.8 \mathrm{~Hz}, 2 \mathrm{H}), 2.14-1.94(\mathrm{~m}, 2 \mathrm{H})$.

${ }^{13}$ C NMR (101 MHz, CDCl $): \delta 167.1,146.7,135.2,133.0,131.1,130.7,130.0,128.6,128.6,128.3,126.0$, $118.9,73.3,52.2$ (splits to doublet), 34.4, 32.0.

HRMS (ESI): Exact mass calculated for $[\mathrm{M}+\mathrm{H}]^{+}\left(\mathrm{C}_{18} \mathrm{H}_{18} \mathrm{IO}_{5}\right) \mathrm{m} / \mathrm{z} 441.0199$, found 441.0203 .

MP: $198-200^{\circ} \mathrm{C}$.

Benziodoxolone 2p<smiles>BrOCCCc1ccccc1Br</smiles>

Following general method B, benziodoxolone $\mathbf{2 p}$ was obtained as white powder in $88 \%$ yield.

${ }^{1} \mathbf{H}$ NMR (400 MHz, $\left.\mathbf{C D C l}_{3}\right): \delta 8.26(\mathrm{dd}, J=7.6,1.5 \mathrm{~Hz}, 0 \mathrm{H}), 7.87(\mathrm{ddd}, J=8.5,7.2,1.6 \mathrm{~Hz}, 0 \mathrm{H}), 7.76(\mathrm{dd}, J=$ $8.3,1.0 \mathrm{~Hz}, 0 \mathrm{H}), 7.68(\mathrm{td}, J=7.4,1.0 \mathrm{~Hz}, 0 \mathrm{H}), 7.59-7.51(\mathrm{~m}, 0 \mathrm{H}), 7.31-7.20(\mathrm{~m}, 1 \mathrm{H}), 7.08(\mathrm{ddd}, J=8.0,5.9$, $3.2 \mathrm{~Hz}, 0 \mathrm{H}), 4.25(\mathrm{t}, J=6.3 \mathrm{~Hz}, 1 \mathrm{H}), 2.97-2.81(\mathrm{~m}, 1 \mathrm{H}), 2.11-1.89(\mathrm{~m}, 1 \mathrm{H})$.

${ }^{13}$ C NMR (101 MHz, CDCl $): \delta 168.0,140.6,135.2,133.1,133.0,131.1,130.8,130.5,128.1,127.8,126.1$, 124.6, 119.0, 73.5, 33.4, 32.5.

HRMS (ESI): Exact mass calculated for $[\mathrm{M}+\mathrm{H}]^{+}\left(\mathrm{C}_{16} \mathrm{H}_{15} \mathrm{BrIO}_{3}\right) \mathrm{m} / \mathrm{z} 460.9237$, found 460.9244 .

MP: $85-87^{\circ} \mathrm{C}$.

\section{Benziodoxolone 2q}<smiles>Clc1ccc(CCCOBr)cc1</smiles>

Following general method B, benziodoxolone $\mathbf{2 q}$ was obtained as white powder in $87 \%$ yield.

${ }^{1}$ H NMR (400 MHz, CDCl $)$ : $\delta 8.27$ (dd, $\left.J=7.6,1.4 \mathrm{~Hz}, 1 \mathrm{H}\right), 7.86$ (ddd, $\left.J=8.5,7.1,1.6 \mathrm{~Hz}, 1 \mathrm{H}\right), 7.76-7.53$ $(\mathrm{m}, 2 \mathrm{H}), 7.27(\mathrm{~d}, J=8.4 \mathrm{~Hz}, 2 \mathrm{H}), 7.14(\mathrm{~d}, J=8.4 \mathrm{~Hz}, 2 \mathrm{H}), 4.21(\mathrm{t}, J=6.4 \mathrm{~Hz}, 2 \mathrm{H}), 2.74(\mathrm{dd}, J=8.4,6.8 \mathrm{~Hz}$, 2H), $2.09-1.90(\mathrm{~m}, 2 \mathrm{H})$.

${ }^{13}$ C NMR (101 MHz, CDCl $): ~ \delta 168.0,139.6,135.2,133.0,132.1,131.2,130.7,129.9,128.8,126.0,118.9$, 73.3, 34.7, 31.3.

HRMS (ESI): Exact mass calculated for $[\mathrm{M}+\mathrm{H}]^{+}\left(\mathrm{C}_{16} \mathrm{H}_{15} \mathrm{ClIO}_{3}\right) \mathrm{m} / \mathrm{z} 416.9750$, found 416.9749 MP: $115-118^{\circ} \mathrm{C}$

$\underline{\text { Benziodoxolone 2r }}$<smiles>BrOC1CCCCC1Cc1ccccc1</smiles>

Following general method B, benziodoxolone $2 \mathbf{r}$ was obtained as white powder in $70 \%$ yield.

${ }^{1}$ H NMR (400 MHz, CDCl 3$): ~ \delta 8.26(\mathrm{dd}, J=7.6,1.6 \mathrm{~Hz}, 1 \mathrm{H}), 7.93(\mathrm{~d}, J=8.2 \mathrm{~Hz}, 1 \mathrm{H}), 7.85$ (ddd, $J=8.4,7.1$, $1.6 \mathrm{~Hz}, 1 \mathrm{H}), 7.67(\mathrm{tt}, J=7.1,1.1 \mathrm{~Hz}, 1 \mathrm{H}), 7.36-7.28(\mathrm{~m}, 2 \mathrm{H}), 7.25-7.16(\mathrm{~m}, 3 \mathrm{H}), 3.68(\mathrm{td}, J=10.2,9.0,4.1$ 
$\mathrm{Hz}, 1 \mathrm{H}), 3.26(\mathrm{dd}, J=13.2,4.1 \mathrm{~Hz}, 1 \mathrm{H}), 2.44(\mathrm{dd}, J=13.2,9.0 \mathrm{~Hz}, 1 \mathrm{H}), 2.14-1.92(\mathrm{~m}, 1 \mathrm{H}), 1.84-1.66(\mathrm{~m}$, $3 \mathrm{H}), 1.63-1.54(\mathrm{~m}, 1 \mathrm{H}), 1.47(\mathrm{td}, J=11.1,10.2,3.4 \mathrm{~Hz}, 1 \mathrm{H}), 1.38-1.24(\mathrm{~m}, 1 \mathrm{H}), 1.20-1.11(\mathrm{~m}, 1 \mathrm{H}), 1.09-$ $0.97(\mathrm{~m}, 1 \mathrm{H})$.

${ }^{13}$ C NMR (101 MHz, CDCl3): $\delta 168.2,140.5,134.9,132.8,131.0,130.8,129.5,128.6,126.7,126.3,120.1$, 85.6, 47.4, 39.5, 35.3, 30.6, 25.2, 25.0.

HRMS (ESI): Exact mass calculated for $[\mathrm{M}+\mathrm{H}]^{+}\left(\mathrm{C}_{20} \mathrm{H}_{22} \mathrm{IO}_{3}\right) \mathrm{m} / \mathrm{z} 437.0623$, found 437.0608 .

MP: $128-130^{\circ} \mathrm{C}$.

$\underline{\text { Benziodoxolone 2s }}$

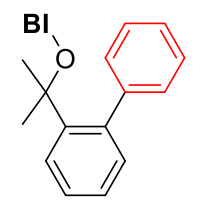

Following general method B, benziodoxolone $2 \mathrm{~s}$ was obtained as white powder in $52 \%$ yield of $75 \%$ purity

${ }^{1}$ H NMR (400 MHz, CDCl 3$): \delta 8.22(\mathrm{dd}, J=7.5,1.6 \mathrm{~Hz}, 1 \mathrm{H}), 7.89(\mathrm{dd}, J=8.3,1.0 \mathrm{~Hz}, 1 \mathrm{H}), 7.80(\mathrm{ddd}, J=8.4$, $7.2,1.6 \mathrm{~Hz}, 1 \mathrm{H}), 7.74-7.71(\mathrm{~m}, 1 \mathrm{H}), 7.62(\mathrm{td}, J=7.3,1.0 \mathrm{~Hz}, 1 \mathrm{H}), 7.44(\mathrm{ddd}, J=8.0,7.3,1.6 \mathrm{~Hz}, 1 \mathrm{H}), 7.38-$ $7.32(\mathrm{~m}, 4 \mathrm{H}), 7.32-7.27(\mathrm{~m}, 2 \mathrm{H}), 7.17$ (dd, $J=7.6,1.6 \mathrm{~Hz}, 1 \mathrm{H}), 1.54(\mathrm{~s}, 6 \mathrm{H})$.

${ }^{13}$ C NMR (101 MHz, $\left.\mathbf{C D C l}_{3}\right): \delta$ 168.0, 144.7, 143.6, 140.9, 136.1, 134.7, 133.2, 133.0, 132.4, 130.8, 130.7, $129.4,128.1,127.7,127.4,127.1,127.0,126.2,119.6,82.2,31.7$.

HRMS (ESI): Exact mass calculated for $[\mathrm{M}+\mathrm{H}]^{+}\left(\mathrm{C}_{22} \mathrm{H}_{20} \mathrm{IO}_{3}\right) \mathrm{m} / \mathrm{z} 459.0464$, found 459.0452 .

MP: $15-160{ }^{\circ} \mathrm{C}$.

\section{$\underline{\text { Benziodoxolone 2t }}$}<smiles>CC(C)(CCc1cccc2ccccc12)OBr</smiles>

Following general method B, benziodoxolone $2 \mathrm{t}$ was obtained as white powder in $68 \%$ yield.

${ }^{1} \mathbf{H}$ NMR (400 MHz, CDCl 3$): \delta 8.26(\mathrm{dd}, J=7.5,1.5 \mathrm{~Hz}, 1 \mathrm{H}), 8.07-8.02(\mathrm{~m}, 1 \mathrm{H}), 7.94(\mathrm{dd}, J=8.2,0.9 \mathrm{~Hz}$, $1 \mathrm{H}), 7.90-7.80(\mathrm{~m}, 2 \mathrm{H}), 7.77-7.72(\mathrm{~m}, 1 \mathrm{H}), 7.66(\mathrm{td}, J=7.4,1.0 \mathrm{~Hz}, 1 \mathrm{H}), 7.57-7.46(\mathrm{~m}, 2 \mathrm{H}), 7.46-7.35$ (m, 2H), $3.44-3.08(\mathrm{~m}, 2 \mathrm{H}), 2.21-1.99(\mathrm{~m}, 2 \mathrm{H}), 1.49(\mathrm{~s}, 6 \mathrm{H})$.

${ }^{13}$ C NMR (101 MHz, CDCl 3$): \delta 168.2,138.0,134.9,134.1,132.6,131.7,131.0,130.9,129.2,127.1,126.5$, $126.2,126.2,125.8,125.8,123.5,119.9,80.4,44.7,28.6,28.2$.

HRMS (ESI): Exact mass calculated for $[\mathrm{M}+\mathrm{H}]^{+}\left(\mathrm{C}_{22} \mathrm{H}_{22} \mathrm{IO}_{3}\right) \mathrm{m} / \mathrm{z} 461.0611$, found 461.0608 . MP: $160-162^{\circ} \mathrm{C}$.

\section{$\underline{\text { Benziodoxolone 2u}}$}<smiles>BrOC(CCc1ccccc1)(c1ccccc1)c1ccccc1</smiles>

Following general method B, benziodoxolone $\mathbf{2 u}$ was obtained as white powder in $54 \%$ yield.

${ }^{1} \mathbf{H}$ NMR (400 MHz, CDCl3): $\delta 8.23(\mathrm{dd}, J=7.6,1.6 \mathrm{~Hz}, 1 \mathrm{H}), 8.15(\mathrm{dd}, J=8.3,0.9 \mathrm{~Hz}, 1 \mathrm{H}), 7.88(\mathrm{ddd}, J=8.3$, $7.2,1.6 \mathrm{~Hz}, 1 \mathrm{H}), 7.65(\mathrm{td}, J=7.4,0.9 \mathrm{~Hz}, 1 \mathrm{H}), 7.51-7.44(\mathrm{~m}, 4 \mathrm{H}), 7.40-7.29(\mathrm{~m}, 6 \mathrm{H}), 7.26-7.12(\mathrm{~m}, 3 \mathrm{H})$, $7.06-6.98(\mathrm{~m}, 2 \mathrm{H}), 2.76-2.69(\mathrm{~m}, 2 \mathrm{H}), 2.56-2.48(\mathrm{~m}, 2 \mathrm{H})$.

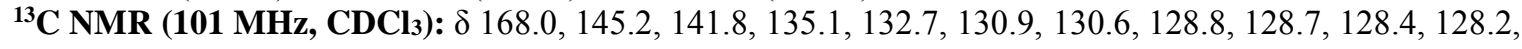
$127.7,127.1,126.3,119.3,85.1,42.9,30.8$.

HRMS (ESI): Exact mass calculated for $[\mathrm{M}+\mathrm{H}]^{+}\left(\mathrm{C}_{28} \mathrm{H}_{24} \mathrm{IO}_{3}\right) \mathrm{m} / \mathrm{z} 535.0692$, found 535.0692 . MP: $156-158^{\circ} \mathrm{C}$. 


\section{CYCLIC VOLTAMMOGRAM}

Cyclic voltammogram of benziodoxolone 3g was collected with a Princeton Applied Research Parstat 4000+ Potentiostat. The sample was prepared with approximately $100 \mu \mathrm{mol}$ of the compound in $5.0 \mathrm{ml}$ of $0.1 \mathrm{M} \mathrm{NBu}_{4} \mathrm{PF}_{6}$ anhydrous acetonitrile and $\mathrm{N}_{2}$ (g) was sparged during 30 minutes. $\mathrm{Ag} / \mathrm{AgNO}_{3}(10 \mu \mathrm{M}$ in $\mathrm{CH} 3 \mathrm{CN})$ was used as a reference electrode. Voltammograms were measured with $100 \mathrm{mV} / \mathrm{s}$ sweep rate. Reductions were measured by scanning potentials in the negative direction. The reference electrode was polished between the runs.

\section{Cyclic volammogram of $\mathbf{2 g}$}

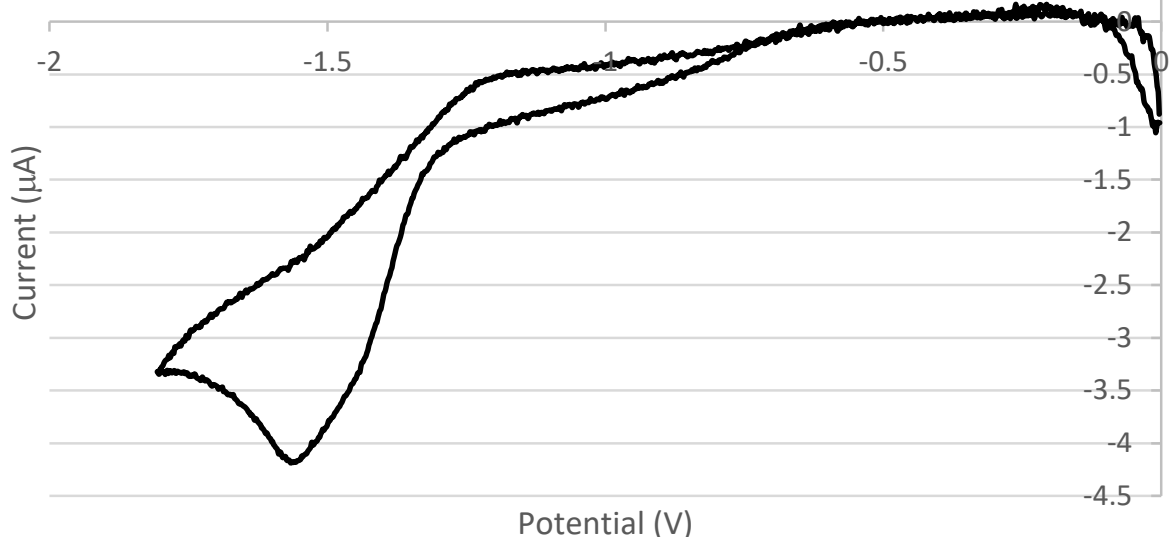

Potential (V) 


\section{PHOTOREDOX CATALYTIC STUDIES}

\subsection{Optimization Studies for 1-5 HAT reactions}

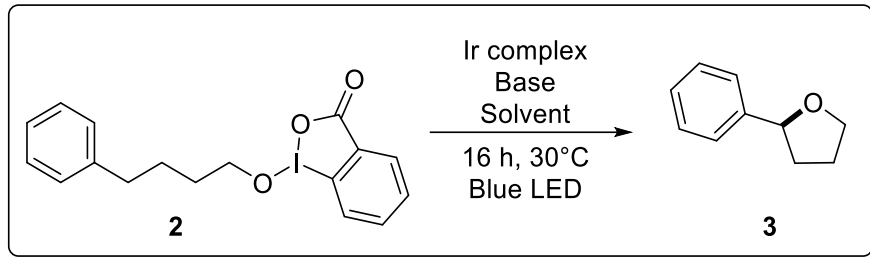

\begin{tabular}{|c|c|c|c|c|c|c|}
\hline Entry & Catalyst & $\mathrm{mol} \%$ & Base & Solvent & Molarity & Yield $^{\mathrm{a}}$ \\
\hline 1 & $\operatorname{Ir}(p p y)_{3}$ & 5 & - & DMF & 0,05 & $0 \%$ \\
\hline 2 & $\operatorname{Ir}(p p y)_{3}$ & 5 & - & DCE & 0,05 & $5 \%$ \\
\hline 3 & $\operatorname{Ir}(\mathrm{ppy})_{3}$ & 5 & - & THF & 0,05 & $5 \%$ \\
\hline 4 & $\operatorname{Ir}(p p y)_{3}$ & 5 & - & $\mathrm{CF}_{3} \mathrm{Ph}$ & 0,05 & $28 \%$ \\
\hline 5 & $\operatorname{Ir}(p p y)_{3}$ & 5 & - & $\mathrm{CH}_{3} \mathrm{CN}$ & 0,05 & $40 \%$ \\
\hline 6 & $\operatorname{Ir}(d F-p p y)_{3}$ & 5 & - & $\mathrm{CH}_{3} \mathrm{CN}$ & 0,05 & $46 \%$ \\
\hline 7 & $\operatorname{Ir}\left(\mathrm{dF}-\mathrm{CF}_{3} \mathrm{ppy}\right)_{2}(\mathrm{dtpy}) \mathrm{PF}_{6}$ & 5 & - & $\mathrm{CH}_{3} \mathrm{CN}$ & 0,05 & $40 \%$ \\
\hline 8 & $\operatorname{Ir}(\mathrm{ppy})_{2}(\mathrm{dtbpy}) \mathrm{BF}_{4}$ & 5 & - & $\mathrm{CH}_{3} \mathrm{CN}$ & 0,05 & $15 \%$ \\
\hline 9 & $\mathrm{Ru}(\mathrm{dtbpy})_{3}\left(\mathrm{PF}_{6}\right)_{2}$ & 5 & - & $\mathrm{CH}_{3} \mathrm{CN}$ & 0,05 & $29 \%$ \\
\hline 10 & 4CzIPN & 5 & - & $\mathrm{CH}_{3} \mathrm{CN}$ & 0,05 & $13 \%$ \\
\hline 11 & Rose Bengal & 5 & - & $\mathrm{CH}_{3} \mathrm{CN}$ & 0,05 & $4 \%$ \\
\hline 12 & Eosin Y & 5 & - & $\mathrm{CH}_{3} \mathrm{CN}$ & 0,05 & $5 \%$ \\
\hline 13 & $\operatorname{Ir}(p p y)_{3}$ & 5 & $\mathrm{~K}_{2} \mathrm{HPO}_{4}$ & $\mathrm{CH}_{3} \mathrm{CN}$ & 0,05 & $42 \%$ \\
\hline 14 & $\operatorname{Ir}(p p y)_{3}$ & 5 & Amberlite & $\mathrm{CH}_{3} \mathrm{CN}$ & 0,05 & $46 \%$ \\
\hline 15 & $\operatorname{Ir}(p p y)_{3}$ & 5 & Pyridine & $\mathrm{CH}_{3} \mathrm{CN}$ & 0,05 & $50 \%$ \\
\hline 16 & $\operatorname{Ir}(\mathrm{ppy})_{3}$ & 5 & Lutidine & $\mathrm{CH}_{3} \mathrm{CN}$ & 0,05 & $35 \%$ \\
\hline 17 & $\operatorname{Ir}(p p y)_{3}$ & 5 & DMAP & $\mathrm{CH}_{3} \mathrm{CN}$ & 0,05 & $32 \%$ \\
\hline 18 & $\operatorname{Ir}(p p y)_{3}$ & 5 & Py & $\mathrm{CH}_{3} \mathrm{CN}$ & 0,10 & $43 \%$ \\
\hline 19 & $\operatorname{Ir}(p p y)_{3}$ & 5 & Py & $\mathrm{CH}_{3} \mathrm{CN}$ & 0,025 & $49 \%$ \\
\hline 20 & $\operatorname{Ir}(p p y)_{3}$ & 2 & Py & $\mathrm{CH}_{3} \mathrm{CN}$ & 0,05 & $44 \%$ \\
\hline 21 & $\operatorname{Ir}(p p y)_{3}$ & 10 & Py & $\mathrm{CH}_{3} \mathrm{CN}$ & 0,05 & $44 \%$ \\
\hline 22 & $\operatorname{Ir}(p p y)_{3}$ & 5 & Py & $\mathrm{CH}_{3} \mathrm{CN}$ & 0,05 & $52 \%{ }^{\mathrm{b}}$ \\
\hline 23 & No catalyst & 0 & Py & $\mathrm{CH}_{3} \mathrm{CN}$ & 0,05 & $0 \%$ \\
\hline 24 & $\operatorname{Ir}(p p y)_{3}$ & 5 & Py & $\mathrm{CH}_{3} \mathrm{CN}$ & 0,025 & $0 \%{ }^{\mathrm{c}}$ \\
\hline
\end{tabular}

Table S1. a) Yield determined by ${ }^{1} \mathrm{H}$ NMR after $16 \mathrm{~h}$ using ethylene carbonate an internal standard, b) Reaction performed during $3 \mathrm{~h}$, c) Reaction performed in the absence of light.

\subsection{Optimization Studies for $1-5 / 6$ addition reactions}

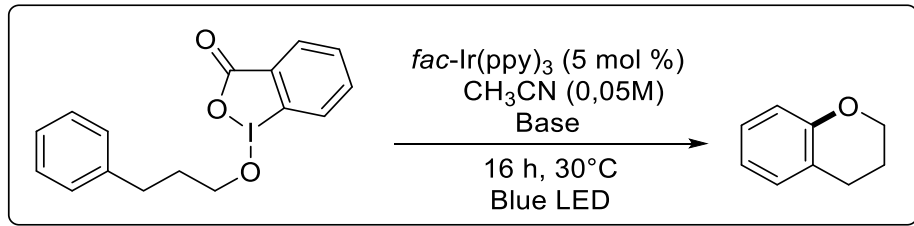

\begin{tabular}{r|c|c|c|c|c|c} 
Entry & Catalyst & mol\% & Base & Solvent & Molarity & Yield \\
\hline 1 & $\mathrm{Ir}(\mathrm{ppy})_{3}$ & 5 & $\mathrm{Py}$ & $\mathrm{CH}_{3} \mathrm{CN}$ & 0,05 & $23 \%^{\mathrm{a}}$ \\
2 & $\mathrm{Ir}(\mathrm{ppy})_{3}$ & 5 & Lutidine & $\mathrm{CH}_{3} \mathrm{CN}$ & 0,05 & $25 \%^{\mathrm{a}}$ \\
3 & $\mathrm{Ir}(\mathrm{ppy})_{3}$ & 5 & - & $\mathrm{CH}_{3} \mathrm{CN}$ & 0,05 & $66 \%^{\mathrm{a}}$ \\
4 & $\mathrm{Ir}(\mathrm{ppy})_{3}$ & 1 & - & $\mathrm{CH}_{3} \mathrm{CN}$ & 0,05 & $25 \%^{\mathrm{a}}$ \\
5 & $\mathrm{No} \mathrm{catalyst}$ & 0 & - & $\mathrm{CH}_{3} \mathrm{CN}$ & 0,05 & $0 \% \%^{\mathrm{a}}$ \\
6 & $\mathrm{Ir}(\mathrm{ppy})_{3}$ & 5 & - & $\mathrm{CH}_{3} \mathrm{CN}$ & 0,05 & $0 \% \%^{\mathrm{a}} \mathrm{b}$
\end{tabular}

Table S2. a) Yield determined by ${ }^{1} \mathrm{H}$ NMR after $16 \mathrm{~h}$ using ethylene carbonate an internal standard, b) Reaction performed in the absence of light. 

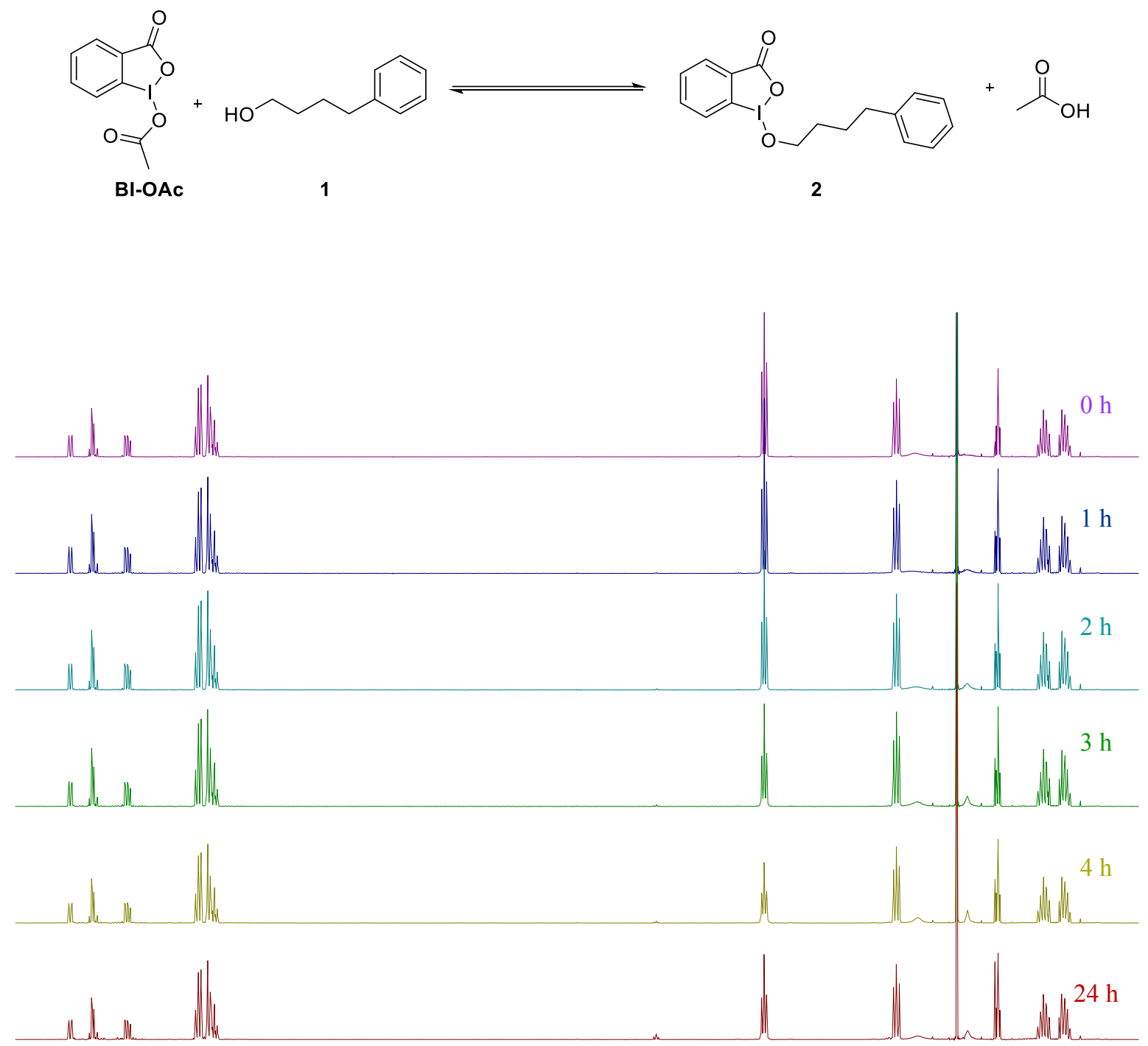

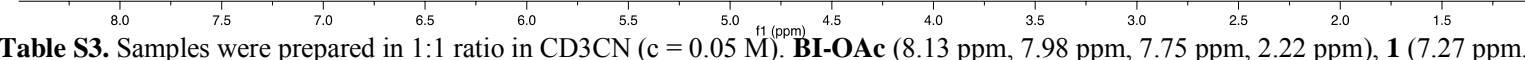
$7.20 \mathrm{ppm}, 3.50 \mathrm{ppm}, 2.62 \mathrm{ppm}, 1,58 \mathrm{ppm}, 1.49 \mathrm{ppm}$,$) , 2$ (4.22 ppm). 

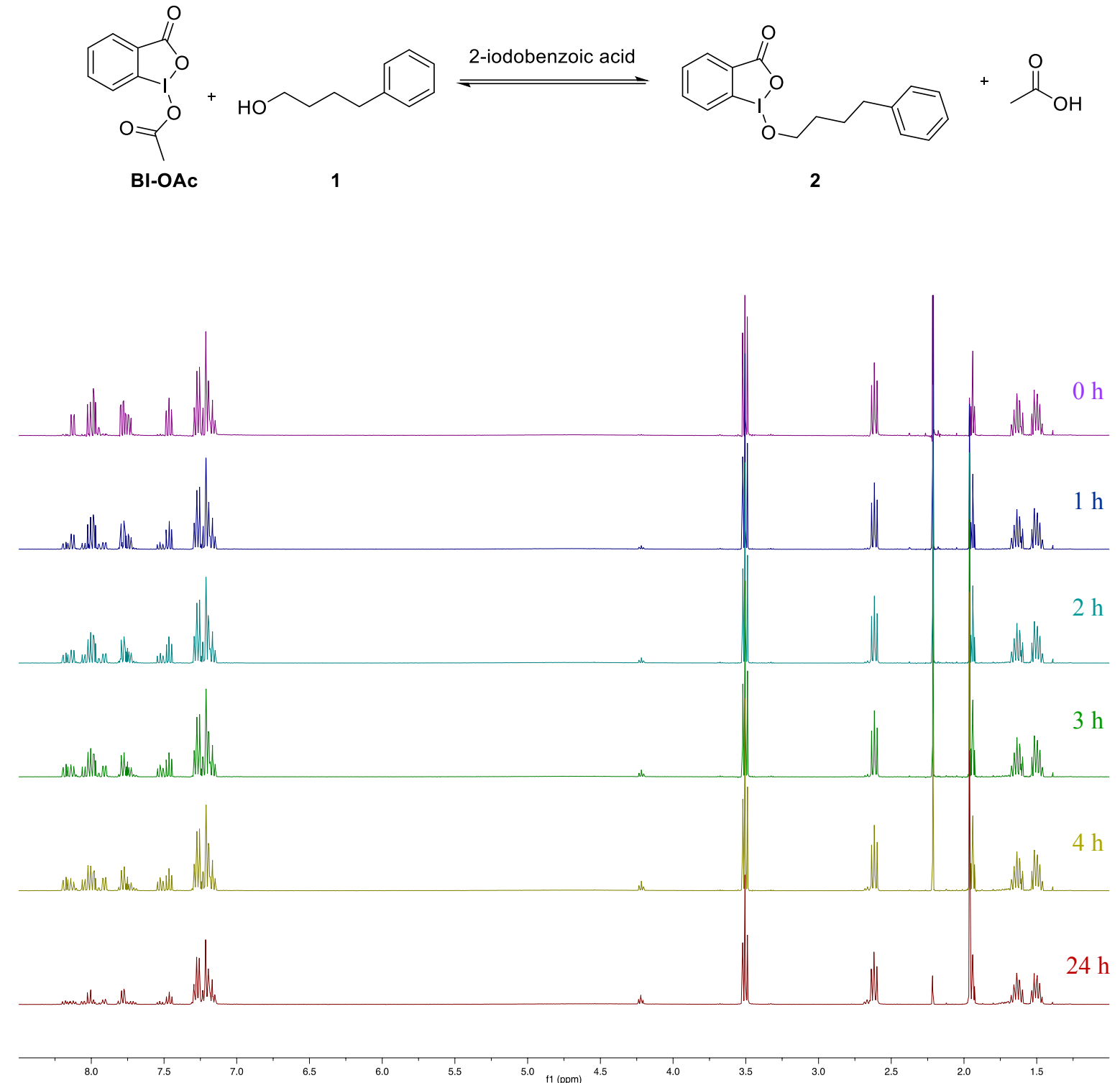

Table S4. Samples were prepared in 1:1:1 ratio in CD3CN $(\mathrm{c}=0.05 \mathrm{M})$. BI-OAc $(8.13 \mathrm{ppm}, 7.98 \mathrm{ppm}, 7.75 \mathrm{ppm}, 2.22 \mathrm{ppm}), \mathbf{1}(7.27 \mathrm{ppm}$, $7.20 \mathrm{ppm}, 3.50 \mathrm{ppm}, 2.62 \mathrm{ppm}, 1,58 \mathrm{ppm}, 1.49 \mathrm{ppm}$,), 2(4.22 ppm).

As shown in Table S3 and S4 (with and without 2-iodobenzoic acid present), only traces of the desired benziodoxolone 2 is formed after 24 hours under the optimized photoredox conditions (acetonitrile as solvent at $\left.30{ }^{\circ} \mathrm{C}\right)$. 
<smiles>O=C1CI(Cl)c2ccccc21</smiles><smiles>O=C(O)c1ccccc1I</smiles><smiles>C1CC1</smiles><smiles>O=C(OI1OC(=O)c2ccccc21)c1ccccc1I</smiles><smiles>OCCCCc1ccccc1</smiles>

2
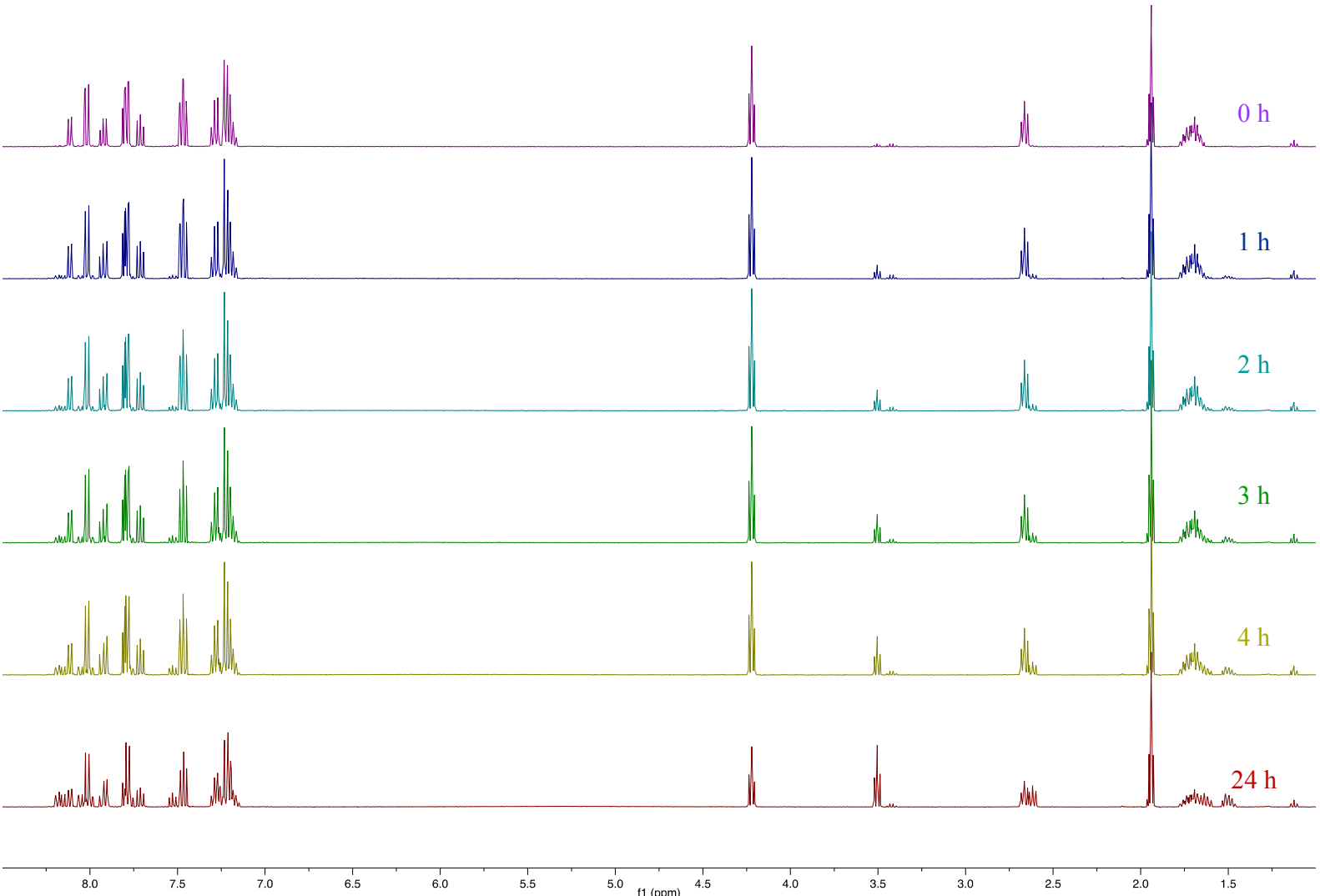

5.5

$5.0^{11(\mathrm{ppm})} 4.5$ 1.5

Table S5. Samples were prepared in 1:1 ratio in $\mathrm{CD} 3 \mathrm{CN}(\mathrm{c}=0.05 \mathrm{M}) . \mathbf{1}(7.27 \mathrm{ppm}, 7.20 \mathrm{ppm}, 3.50 \mathrm{ppm}, 2.62 \mathrm{ppm}, 1,58 \mathrm{ppm}, 1.49 \mathrm{ppm}$,), $\underline{\mathbf{2}}$ (4.22 ppm, $2.66 \mathrm{ppm}, 1.71 \mathrm{ppm})$.

As shown in Table S5, alcohol $\mathbf{1}$ is formed by substitution on benziodoxolone $\mathbf{2}$ with formed iodobenzoic acid under the optimized photoredox conditions (acetonitrile as solvent at $30^{\circ} \mathrm{C}$ ). 
5.5. By-products formation analysis of the photocatalytic etherification.

a)<smiles>COCCCCc1ccccc1</smiles>

fac-Ir(ppy) $)_{3}$ $\mathrm{Py}, \mathrm{CH}_{3} \mathrm{CN}$

$3 \mathrm{~h}, 30^{\circ} \mathrm{C}$ Blue LEDs<smiles></smiles><smiles>C[Te]CCC[Te]</smiles><smiles>c1ccc2c(c1)CCCO2</smiles>

b)<smiles></smiles><smiles>Cc1ccccc1Cc1c(C)cccc1C(=O)O</smiles><smiles>c1ccc(C2CCCCC2)cc1</smiles><smiles>O=C(On1oc(=O)c2ccccc21)c1ccccc1I</smiles>

Scheme S1. Photocatalytic etherification byproducts.

Together with THF derivative 3, compounds 1, 4 and $\mathbf{5}$ were detected in approximately $20 \%, 15 \%$ and $10 \%$ yield, respectively (Scheme S1a). The formation of these byproducts can be rationalized from competing $\mathrm{H}$-atom abstractions other than the 1,5-HAT (compound $\mathbf{1}$ and 4) and via radical addition on to the aromatic moiety (compound 5). Furthermore, we could show that $\mathbf{2}$ is susceptible towards substitution with o-iodobenzoic acid, which is formed during the course of the reaction, producing dimer 6 and alcohol 1 (Scheme S1b). This compound have a redox potential akin to BIOAc and can thus possibly be engaged reductively by the catalyst, generating the corresponding acyloxy radical. Such a radical specie would also engage 2 oxidativly to produce 4 . Taken together, the extent of these side reactions are most probably effected by the characteristics of the benziodoxolone substrate, which is reflected in the different yields obtained for the various substrates investigated 


\section{PROCEDURES AND CHARACTERIZATION OF ETHERS}

\subsection{General method $\mathbf{C}$ for 1-5 HAT reactions}

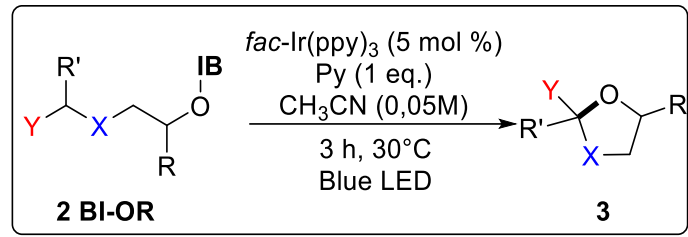

To an oven-dried microwave tube provided with a magnetic stir bar, were added the corresponding benziodoxolone 2 (0.5 mmol, 1 eq.) and $\operatorname{Ir}(\text { ppy })_{3}(16.4 \mathrm{mg}, 0.025 \mathrm{mmol}, 5 \mathrm{~mol} \%)$, after three cycles of vacuum- $\mathrm{N}_{2}, 10 \mathrm{~mL}$ of dry, degassed (Freeze-Pump-Thaw) acetonitrile was added followed by pyridine ( $40 \mu \mathrm{L}, 0.5 \mathrm{mmol}, 1$ eq.). The resulting mixture was stirred under blue light irradiation (placed approximately $2 \mathrm{~cm}$ from the lamps) for $3 \mathrm{~h}$ with an $\mathrm{N}_{2}$ positive pressure. After that time, the solvent was removed in vacuo (except for compounds $\mathbf{3 g}, \mathbf{3 h}$ and $\mathbf{3 k}$, that were poured into $10 \mathrm{~mL}$ of saturated $\mathrm{NaHCO}_{3}$ and extracted with ether to avoid co-distillation with the acetonitrile due to their low boiling point) giving rise to a brown oil. Purification of the crude product by column chromatography on silica gel (eluting with pentane to pentane/ether 95/5) afforded the corresponding ether 3.

\subsection{General method D for $1-5 / 6$ Addition reactions}

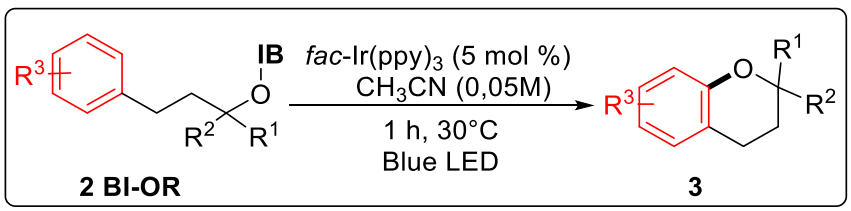

To an oven-dried microwave tube provided with a magnetic stir bar, were added the corresponding benziodoxolone 2 (0.5 mmol, 1 eq.) and $\operatorname{Ir}(\text { ppy })_{3}(16.4 \mathrm{mg}, 0.025 \mathrm{mmol}, 5 \mathrm{~mol} \%)$, after three cycles of vacuum- $\mathrm{N}_{2}, 10 \mathrm{~mL}$ of dry, degassed (Freeze-Pump-Thaw) acetonitrile was added. The resulting mixture was stirred under blue light irradiation (placed approximately $2 \mathrm{~cm}$ from the lamps) for $1 \mathrm{~h}$ with an $\mathrm{N}_{2}$ positive pressure. After that time, the solvent was removed in vасио giving rise to a brown oil. Purification of the crude product by column chromatography on silica gel (eluting with pentane to pentane/ether 95/5) afforded the corresponding ether 3.

\subsection{Characterization of alkyl ethers}

$\underline{\text { 2-phenyl-1,3-dioxolane, ether } \mathbf{3 a}}{ }^{19}$<smiles>c1ccc(C2OCCO2)cc1</smiles>

Following general method $\mathrm{C}$, ether $\mathbf{3 a}$ was obtained as colourless oil in $75 \%$ yield $(0.38 \mathrm{mmol}, 56 \mathrm{mg})$.

${ }^{1}$ H NMR (400 MHz, CDCl 3$): \delta 7.54-7.45(\mathrm{~m}, 2 \mathrm{H}), 7.42-7.36(\mathrm{~m}, 3 \mathrm{H}), 5.83(\mathrm{~s}, 1 \mathrm{H}), 4.19-4.10(\mathrm{~m}, 2 \mathrm{H})$, $4.09-4.00(\mathrm{~m}, 2 \mathrm{H})$.

${ }^{13}$ C NMR (101 MHz, CDCl 3$): \delta 138.0,129.3,128.5,126.6,103.9,65.5$.

\footnotetext{
${ }^{19}$ Kurihara, M.; Hakamata, W. J. Org. Chem. 2003, 68, 3413.
} 
<smiles>COc1ccc(C2OCCO2)cc1</smiles>

Following general method $\mathrm{C}$, ether $\mathbf{3 b}$ was obtained as colourless oil in $92 \%$ yield $(0.46 \mathrm{mmol}, 83 \mathrm{mg})$.

${ }^{1} \mathbf{H}$ NMR (400 MHz, $\left.\mathbf{C D C l}_{3}\right): \delta 7.41(\mathrm{~d}, J=8.4 \mathrm{~Hz}, 2 \mathrm{H}), 6.91(\mathrm{~d}, J=8.8 \mathrm{~Hz}, 2 \mathrm{H}), 5.76(\mathrm{~s}, 1 \mathrm{H}), 4.18-4.07$ (m, $2 \mathrm{H}), 4.09-3.95(\mathrm{~m}, 2 \mathrm{H}), 3.81(\mathrm{~s}, 3 \mathrm{H})$.

${ }^{13}$ C NMR (101 MHz, CDCl3): $\delta$ 160.5, 130.1, 128.0, 113.9, 103.8, 65.4, 55.4 .

$\underline{\text { 2-(2,6-dichlorophenyl)-1,3-dioxolane, ether } 3 \mathbf{c}^{20}}$<smiles>Clc1cccc(Cl)c1C1OCCO1</smiles>

Following general method $\mathrm{C}$, ether $\mathbf{3 c}$ was obtained as colourless solid in $91 \%$ yield $(0.46 \mathrm{mmol}, 100 \mathrm{mg})$.

${ }^{1}$ H NMR (400 MHz, CDCl $): \delta 7.35-7.28(\mathrm{~m}, 2 \mathrm{H}), 7.21(\mathrm{dd}, J=8.8,7.2 \mathrm{~Hz}, 1 \mathrm{H}), 6.48(\mathrm{~s}, 1 \mathrm{H}), 4.37-4.24(\mathrm{~m}$, $2 \mathrm{H}), 4.14-4.00(\mathrm{~m}, 2 \mathrm{H})$.

${ }^{13}$ C NMR (101 MHz, CDCl $): \delta$ 136.2, 131.0, 130.8, 129.6, 101.2, 66.1.

$\underline{\text { 2-phenoxytetrahydrofuran, ether } \mathbf{3 d}^{21}}$<smiles>c1ccc(OC2CCCO2)cc1</smiles>

Following general method C, ether 3d was obtained as colourless oil in $52 \%$ yield $(0.26 \mathrm{mmol}, 43 \mathrm{mg})$.

${ }^{1}$ H NMR (400 MHz, CDCl 3$): \delta 7.33-7.22(\mathrm{~m}, 2 \mathrm{H}), 7.08-7.00(\mathrm{~m}, 2 \mathrm{H}), 7.02-6.93(\mathrm{~m}, 1 \mathrm{H}), 4.09-4.00(\mathrm{~m}$, $1 \mathrm{H}), 4.00-3.90(\mathrm{~m}, 1 \mathrm{H}), 2.24-2.07(\mathrm{~m}, 3 \mathrm{H}), 2.01-1.90(\mathrm{~m}, 1 \mathrm{H})$.

${ }^{13}$ C NMR (101 MHz, CDCl $)$ : $\delta 157.3,129.5,121.6,116.7,102.4,68.2,32.8,23.6$.

$\underline{1,4,6 \text {-trioxaspiro[4.5]decane, ether } 3 \mathbf{e}^{22}}$<smiles>C1CCC2(OC1)OCCO2</smiles>

Following general method $\mathrm{C}$, ether $3 \mathbf{e}$ was obtained as colourless oil in $46 \%$ yield $(0.23 \mathrm{mmol}, 33 \mathrm{mg})$.

${ }^{1} \mathbf{H}$ NMR (400 MHz, CDCl 3$): ~ \delta 4.15-4.05(\mathrm{~m}, 2 \mathrm{H}), 4.04-3.94(\mathrm{~m}, 2 \mathrm{H}), 3.86-3.74(\mathrm{~m}, 2 \mathrm{H}), 1.83(\mathrm{~m}, 4 \mathrm{H})$, $1.58-1.50(\mathrm{~m}, 2 \mathrm{H})$.

${ }^{13}$ C NMR (101 MHz, CDCl $): \delta$ 119.1, 64.9, 64.1, 32.0, 24.7, 21.7.

2-phenyltetrahydrofuran, ether $\mathbf{3}^{23}$<smiles>c1ccc(C2CCCO2)cc1</smiles>

Following general method C, ether $\mathbf{3 f}$ was obtained as colourless oil in $48 \%$ yield $(0.24 \mathrm{mmol}, 36 \mathrm{mg})$.

\footnotetext{
${ }^{20}$ Ramanathan, M.; Hou, D. Arkivoc 2013, 98.

${ }^{21}$ Baati, R.; Valleix, A; Mioskowski, C; Barma D. K.; Falck, J. R. Org. Lett.2000, 2, 485.

${ }^{22}$ La Cruz, T. E.; Rychnovsky, S. D. Synlett 2004, 11, 2013.

${ }^{23}$ Liu, D.; Liu, C.; Li, H.; Lei, A., Angew. Chem., Int. Ed. 2013, 52, 4453.
} 
$\mathbf{1}$ mmol, scale: To an oven-dried Schlenk tube provided with a magnetic stir bar, were added benziodoxolone $2 \mathbf{2 f}$ (1.0 mmol, $396.2 \mathrm{mg}, 1$ eq.) and $\operatorname{Ir}(\text { ppy })_{3}(0.05 \mathrm{mmol}, 3.8 \mathrm{mg}, 5 \mathrm{~mol} \%)$, after three cycles of vacuum- $\mathrm{N}_{2}, 20 \mathrm{~mL}$ of dry, degassed (Freeze-Pump-Thaw) acetonitrile was added followed by pyridine ( $80 \mu \mathrm{L}, 1.0 \mathrm{mmol}, 1$ eq.). The resulting mixture was stirred under blue light irradiation (placed approximately $2 \mathrm{~cm}$ from the lamps) for $3 \mathrm{~h}$ with an $\mathrm{N}_{2}$ positive pressure. After that time, the solvent was removed in vacuo giving rise to a brown oil. Purification of the crude product by column chromatography on silica gel (eluting with pentane to pentane/ether 95/5) afforded the corresponding ether $\mathbf{3 f}$ as colourless oil in $43 \%$ yield $(0.43 \mathrm{mmol}, 64 \mathrm{mg})$.

${ }^{1}$ H NMR (400 MHz, CDCl3): $\delta 7.34-7.30(\mathrm{~m}, 4 \mathrm{H}), 7.27-7.20(\mathrm{~m}, 1 \mathrm{H}), 4.89(\mathrm{t}, J=7.2 \mathrm{~Hz}, 1 \mathrm{H}), 4.17-4.03$ (m, 1H), $3.99-3.83(\mathrm{~m}, 1 \mathrm{H}), 2.36-2.25(\mathrm{~m}, 1 \mathrm{H}), 2.02-1.93(\mathrm{~m}, 2 \mathrm{H}), 1.84-1.72(\mathrm{~m}, 1 \mathrm{H})$.

${ }^{13}$ C NMR (101 MHz, CDCl3): $\delta{ }^{13} \mathrm{C}$ NMR $\left(101 \mathrm{MHz}, \mathrm{cdcl}_{3}\right) \delta 143.6,128.4,127.2,125.8,80.8,68.8,34.8,26.2$.

\section{2-(4-fluorophenyl)tetrahydrofuran, ether $\mathbf{3 g}^{23}$}<smiles>Fc1ccc(C2CCCO2)cc1</smiles>

Following general method $\mathrm{C}$, ether $\mathbf{3 g}$ was obtained as colourless oil in $51 \%$ yield $(0.26 \mathrm{mmol}, 42 \mathrm{mg})$.

${ }^{1}$ H NMR (400 MHz, CDCl $)$ : $\delta 7.40-7.22(\mathrm{~m}, 2 \mathrm{H}), 7.13-6.94(\mathrm{~m}, 2 \mathrm{H}), 4.85(\mathrm{t}, J=7.2 \mathrm{~Hz}, 1 \mathrm{H}), 4.09$ (dt, $J=$ $8.3,6.8 \mathrm{~Hz}, 1 \mathrm{H}), 3.92(\mathrm{~m}, 1 \mathrm{H}), 2.31(\mathrm{td}, J=12.4,6.8 \mathrm{~Hz}, 1 \mathrm{H}), 2.12-1.93(\mathrm{~m}, 2 \mathrm{H}), 1.88-1.72(\mathrm{~m}, 1 \mathrm{H})$.

${ }^{13}$ C NMR (101 MHz, CDCl $): \delta 162.1(\mathrm{~d}, J=244.7 \mathrm{~Hz}), 139.2(\mathrm{~d}, J=3.1 \mathrm{~Hz}), 127.4(\mathrm{~d}, J=8.0 \mathrm{~Hz}), 115.2(\mathrm{~d}, J$ $=21.3 \mathrm{~Hz}), 80.3,68.8,34.8,26.1$.

\section{2-phenyloctahydrobenzofuran, ether $\mathbf{3 h}^{24}$}<smiles>c1ccc(C2CC3CCCCC3O2)cc1</smiles>

Following general method $\mathrm{C}$, ether $\mathbf{3 h}$ was obtained as colourless oil in $97 \%$ yield $(0.49 \mathrm{mmol}, 98 \mathrm{mg})$ as mixture of diastereomers (dr-1:1).

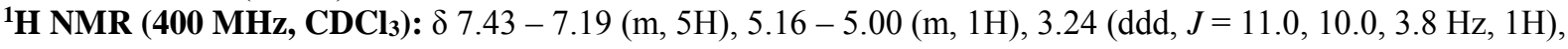
$2.30-2.19(\mathrm{~m}, 1 \mathrm{H}), 2.09-1.85(\mathrm{~m}, 4 \mathrm{H}), 1.771 .10(\mathrm{~m}, 6 \mathrm{H})$.

${ }^{13}$ C NMR (101 MHz, CDCl $)$ : $\delta$ 145.1, 128.5, 127.0, 125.9, 84.5, 78.9, 44.1, 40.0, 31.7, 29.1, 25.8, 24.5.

\section{2,5-diphenyltetrahydrofuran, ether $\mathbf{3 i}^{25}$}<smiles>c1ccc(C2CCC(c3ccccc3)O2)cc1</smiles>

Following general method $\mathrm{C}$, ether $3 \mathbf{i}$ was obtained as colourless oil in $56 \%$ yield $(0.28 \mathrm{mmol}, 63 \mathrm{mg})$ as mixture of diastereomers (dr-1:1).

${ }^{1}$ H NMR (400 MHz, CDCl3) Trans Isomer: $\delta 7.44-7.40(\mathrm{~m}, 4 \mathrm{H}), 7.36(\mathrm{t}, J=7.6 \mathrm{~Hz}, 4 \mathrm{H}), 7.29-7.24(\mathrm{~m}$, $2 \mathrm{H}), 5.31-5.23(\mathrm{~m}, 2 \mathrm{H}), 2.52-2.42(\mathrm{~m}, 2 \mathrm{H}), 2.08-1.93(\mathrm{~m}, 2 \mathrm{H})$.

${ }^{13}$ C NMR (101 MHz, CDCl $)$ Trans Isomer: $\delta 143.8,128.5,127.3,125.7,81.5,35.7$.

${ }^{1}$ H NMR (400 MHz, CDCl$)$ Cis Isomer: $\delta 7.47-7.43(\mathrm{~m}, 4 \mathrm{H}), 7.39-7.33(\mathrm{~m}, 4 \mathrm{H}), 7.31-7.26(\mathrm{~m}, 2 \mathrm{H}), 5.06$ (ddd, $J=7.0,4.7,1.6 \mathrm{~Hz}, 2 \mathrm{H}), 2.52-2.36(\mathrm{~m}, 2 \mathrm{H}), 2.04-1.92(\mathrm{~m}, 2 \mathrm{H})$.

${ }^{13}$ C NMR (101 MHz, CDCl $)$ Cis Isomer: $\delta$ 143.1, 128.5, 127.4, 126.1, 81.4, 34.5.

\footnotetext{
${ }^{24}$ Gharpure, S. J.; Vishwakarma, D. S.; Nanda, S. K. Org. Lett. 2017, 19, 6534.

${ }^{25}$ Imada, Y.; Okada, Y.; Noguchi, K.; Chiba, Z. Angew. Chem., Int. Ed. 2019, 58, 125.
} 


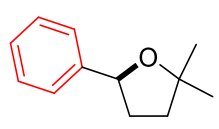

Following general method $\mathrm{C}$, ether $\mathbf{3 j}$ was obtained as colourless oil in $56 \%$ yield $(0.28 \mathrm{mmol}, 49 \mathrm{mg})$.

${ }^{1}$ H NMR (400 MHz, CDCl 3$): \delta 7.38-7.28(\mathrm{~m}, 4 \mathrm{H}), 7.27-7.20(\mathrm{~m}, 1 \mathrm{H}), 5.03-4.95(\mathrm{~m}, 1 \mathrm{H}), 2.39-2.27(\mathrm{~m}$, $1 \mathrm{H}), 1.97-1.83(\mathrm{~m}, 3 \mathrm{H}), 1.40(\mathrm{~s}, 3 \mathrm{H}), 1.36(\mathrm{~s}, 3 \mathrm{H})$.

${ }^{13}$ C NMR (101 MHz, CDCl $)$ : $\delta$ 143.6, 128.2, 127.1, 125.8, 81.3, 80.5, 39.0, 35.6, 29.0, 28.4.

1,1-dimethyl-3-phenyl-1,3-dihydroisobenzofuran, ether 3k<smiles>CC1(C)OC(c2ccccc2)c2ccccc21</smiles>

Following general method C, ether $3 \mathbf{k}$ was obtained as colourless oil in $90 \%$ yield $(0.45 \mathrm{mmol}, 101 \mathrm{mg})$.

${ }^{1}$ H NMR (400 MHz, CDCl $): \delta 7.38-7.27(\mathrm{~m}, 6 \mathrm{H}), 7.24-7.15(\mathrm{~m}, 2 \mathrm{H}), 6.96(\mathrm{dd}, J=7.5,1.0 \mathrm{~Hz}, 1 \mathrm{H}), 6.15(\mathrm{~s}$, $1 \mathrm{H}), 1.70(\mathrm{~s}, 3 \mathrm{H}), 1.56(\mathrm{~s}, 3 \mathrm{H})$.

${ }^{13} \mathbf{C}$ NMR (101 MHz, CDCl $)$ : $\delta 147.2,142.3,141.6,128.6,128.1,127.9,127.6,127.5,122.5,120.5,85.7,83.9$, 83.9, 29.5, 29.0.

HRMS (ESI): Exact mass calculated for $[\mathrm{M}+\mathrm{H}]^{+}\left(\mathrm{C}_{16} \mathrm{H}_{17} \mathrm{O}\right) \mathrm{m} / \mathrm{z} 225.1279$, found 225.1274 .

\subsection{Characterization of alryl ethers}

Chromane, ether $\mathbf{3 1}^{27}$<smiles>c1ccc2c(c1)CCCO2</smiles>

Following general method D, ether $3 \mathbf{l}$ was obtained as colourless oil in $55 \%$ yield $(0.28 \mathrm{mmol}, 37 \mathrm{mg})$.

${ }^{1} \mathbf{H}$ NMR (400 MHz, CDCl 3$): \delta 7.14-6.98(\mathrm{~m}, 2 \mathrm{H}), 6.92-6.71(\mathrm{~m}, 2 \mathrm{H}), 4.28-4.08(\mathrm{~m}, 2 \mathrm{H}), 2.80(\mathrm{t}, J=6.5$ $\mathrm{Hz}, 2 \mathrm{H}), 2.07-1.92(\mathrm{~m}, 2 \mathrm{H})$.

${ }^{13}$ C NMR (101 MHz, CDCl $): \delta 155.0,129.9,127.3,122.4,120.2,116.8,66.6,25.0,22.5$.

\section{$\underline{6,7,8 \text {-trimethoxychromane, ether } 3 \mathbf{m}^{28}}$}<smiles>COc1cc2c(c(OC)c1OC)OCCC2</smiles>

Following general method D, ether $\mathbf{3 m}$ was obtained as colourless oil in $56 \%$ yield $(0.28 \mathrm{mmol}, 63 \mathrm{mg})$.

${ }^{1}$ H NMR (400 MHz, CDCl$): \delta 6.33(\mathrm{~s}, 1 \mathrm{H}), 4.26-4.13(\mathrm{~m}, 2 \mathrm{H}), 3.89(\mathrm{~s}, 3 \mathrm{H}), 3.87(\mathrm{~s}, 3 \mathrm{H}), 3.79(\mathrm{~s}, 3 \mathrm{H}), 2.73$ (t, $J=6.5 \mathrm{~Hz}, 2 \mathrm{H}), 2.06-1.89(\mathrm{~m}, 2 \mathrm{H})$.

${ }^{13}$ C NMR (101 MHz, CDCl 3 ): $\delta .146 .7,142.7,142.4,141.5,117.2,107.6,66.5,61.4,61.3,56.5,24.9,22.5$.

$\underline{5-m e t h o x y c h r o m a n e ~ a n d ~ 8-m e t h o x y c h r o m a n e, ~ e t h e r s ~} \mathbf{3 n}$ and $\mathbf{3 n} \mathbf{n}^{29}$

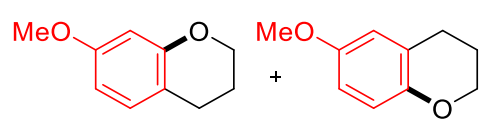

\footnotetext{
${ }^{26}$ Kundu, R.; Ball, Z. T. Org. Lett.2010, 12, 2460.

${ }^{27}$ Shi, Z.; He, C. J. Am. Chem. Soc. 2004, 126, 13596.

${ }^{28}$ Henry, M. C.; Senn, H. M.; Sutherland, A. J. Org. Chem. 2019, 84, 346.

${ }^{29}$ Houry, S.; Geresh, S.; Shani, A. Isr. J. Chem. 1973, 11, 805.
} 
Following general method D, ethers 3n and 3n' were obtained as an inseparable mixture, colourless oil in $44 \%$ yield $(0.22 \mathrm{mmol}, 36 \mathrm{mg})$ in $3: 1$ ratio.

${ }^{1}$ H NMR (400 MHz, CDCl3): $\delta 77.16-7.06(\mathrm{~m}, 1 \mathrm{H}), 6.92-6.78(\mathrm{~m}, 1 \mathrm{H}), 6.71(\mathrm{~d}, J=2.2 \mathrm{~Hz}, 0 \mathrm{H}), 6.68-6.65$ $(\mathrm{m}, 0 \mathrm{H}), 6.60-6.55(\mathrm{~m}, 0 \mathrm{H}), 4.13(\mathrm{dd}, J=5.8,4.5 \mathrm{~Hz}, 1 \mathrm{H}), 3.88(\mathrm{t}, J=6.3 \mathrm{~Hz}, 1 \mathrm{H}), 3.79(\mathrm{~s}, 1 \mathrm{H}), 3.75(\mathrm{~s}, 0 \mathrm{H})$, $2.92-2.63(\mathrm{~m}, 1 \mathrm{H}), 2.12-1.89(\mathrm{~m}, 1 \mathrm{H})$.

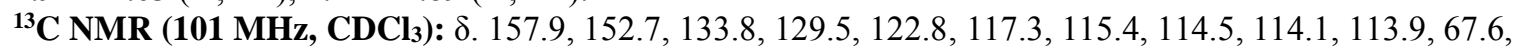
$66.5,55.9,55.4,31.4,31.3,25.3,22.6$.

HRMS (ESI): Exact mass calculated for $[\mathrm{M}+\mathrm{H}]^{+}\left(\mathrm{C}_{10} \mathrm{H}_{13} \mathrm{O}_{2}\right) \mathrm{m} / \mathrm{z} 165.0915$, found 165.0912.

$\underline{\text { 5-methoxychromane and 8-methoxychromane, ethers } \mathbf{3 0}^{30} \text { and } \mathbf{3 0}^{, 31}}$<smiles>CC(=O)c1ccc2c(c1)OCCC2</smiles>

Following general method D, ethers 3o and 3o' were obtained as an inseparable mixture, colourless oil in $37 \%$ $(0.19 \mathrm{mmol}, 36 \mathrm{mg})$ yield in 5:1 ratio.

${ }^{1}$ H NMR (400 MHz, CDCl 3$): \delta 7.89-7.65$ (m, 2H, minor), 7.49 (dd, $J=7.9,1.7 \mathrm{~Hz}, 7 \mathrm{H}$, major), $7.45(\mathrm{dt}, J=$ $1.8,0.4 \mathrm{~Hz}, 7 \mathrm{H}$, major), $7.11-7.05(\mathrm{~m}, 7 \mathrm{H}$, major), 6.79 (d, $J=9.1 \mathrm{~Hz}, 1 \mathrm{H}$, minor), $4.27-4.20$ (m, 2H, minor), $4.22-4.17$ (m, 14H, major), $3.88(\mathrm{~s}, 21 \mathrm{H}$, major), 3.87 (s, 3H, minor), $2.86-2.75(\mathrm{~m}, 18 \mathrm{H}$, minor + major $)$, $2.14-1.87(\mathrm{~m}, 19 \mathrm{H}$, minor + major $)$.

${ }^{13}$ C NMR (101 MHz, CDCl3): $\delta .167 .2$ (minor), 167.1 (major), 155.0 (major), 155.0 (major), 132.0 (minor), 129.9 (major), 129.4 (major), 129.2 (minor), 127.8 (major), 122.0 (minor), 121.2 (major), 118.1 (major), 116.8 (major), 67.0 (minor), 66.63 (major), 52.1 (major), 51.9 (minor), 25.2 (major), 24.9 (minor), 22.1 (minor).

5-bromochromane and 8-bromochromane, ethers $\mathbf{3 p}$ and $\mathbf{3} \mathbf{p}^{, 32}$<smiles>Brc1cccc2c1CCCO2</smiles>

Following general method D, ethers 3p and 3p' were obtained as an inseparable mixture, colourless oil in 52\% yield $(0.26 \mathrm{mmol}, 55 \mathrm{mg})$ in $1: 1$ ratio.

${ }^{1} \mathbf{H}$ NMR (400 MHz, CDCl$): \delta 7.37-7.30(\mathrm{~m}, 1 \mathrm{H}), 7.11(\mathrm{dd}, J=7.9,1.2 \mathrm{~Hz}, 1 \mathrm{H}), 7.05-6.90(\mathrm{~m}, 2 \mathrm{H}), 6.80-$ $6.73(\mathrm{~m}, 1 \mathrm{H}), 6.71(\mathrm{t}, J=7.7 \mathrm{~Hz}, 1 \mathrm{H}), 4.41-4.24(\mathrm{~m}, 2 \mathrm{H}), 4.21-4.08(\mathrm{~m}, 2 \mathrm{H}), 2.81(\mathrm{t}, J=6.5 \mathrm{~Hz}, 2 \mathrm{H}), 2.76(\mathrm{t}$, $J=6.6 \mathrm{~Hz}, 2 \mathrm{H}), 2.08-1.97(\mathrm{~m}, 4 \mathrm{H})$.

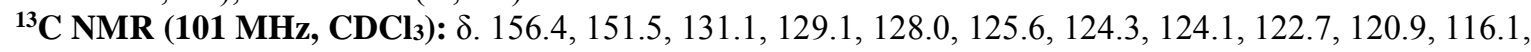
$111.0,67.5,66.3,26.1,25.3,22.5,22.2$.

7-chlorochromane and 6-chlorochromane, ethers $\mathbf{3 q}$ and $\mathbf{3 q} \mathbf{q}^{32}$<smiles>Clc1ccc2c(c1)OC[CH-]C2</smiles>

Following general method D, ethers $\mathbf{3 q}$ and $\mathbf{3 q}$ ' were obtained as an inseparable mixture, colourless oil in 54\% $(0.27 \mathrm{mmol}, 46 \mathrm{mg})$ yield in $1: 1$ ratio.

${ }^{1}$ H NMR (400 MHz, CDCl $): \delta{ }^{1} \mathrm{H}$ NMR (400 MHz, Chloroform- $d$ ) $\delta 7.06-6.90(\mathrm{~m}, 3 \mathrm{H}), 6.85-6.65(\mathrm{~m}, 3 \mathrm{H})$, $4.23-4.09(\mathrm{~m}, 4 \mathrm{H}), 2.75(\mathrm{q}, J=6.6 \mathrm{~Hz}, 4 \mathrm{H}), 2.08-1.92(\mathrm{~m}, 4 \mathrm{H})$.

${ }^{13}$ C NMR (101 MHz, CDCl $): \delta .155 .5,153.5,132.1,130.6,129.3,127.1,124.7,123.7,120.7,120.2,118.0$, $116.8,66.5,66.5,24.8,24.4,22.1,22.0$.

\footnotetext{
${ }^{30}$ Walker, D. P.; Piotrowski, D. W.; Jacobsen, E. J.; Acker, B. A.; Groppi, V. E. Azabicyclic-phenyl-fused-heterocyclic compounds for treatment of diseases. U.S. Patent 2003130305A1, July 10, 2003.

${ }^{31}$ Geist, E.; Berneaud-Kötz, H.; Baikstis, T.; Dräger, G.; Kirschning, A. Org. Lett. 2019, 21, $22,8930$.

${ }^{32}$ Zheng, Y.; Ye, P.; Chen, B; Meng, Q.; Feng, K.; Wang, W.; Wu, L.; Tung. C. Org. Lett. 2017, 19, 2206.
} 
<smiles>c1ccc2c(c1)CC1CCCCC1O2</smiles>

Following general method D, ether $3 \mathbf{r}$ was obtained as white crystals in $50 \%$ yield $(0.25 \mathrm{mmol}, 47 \mathrm{mg})$.

${ }^{1} \mathbf{H}$ NMR (400 MHz, CDCl 3$)$ : $\delta{ }^{1} \mathrm{H}$ NMR (400 MHz, Chloroform- $d$ ) $\delta 7.13-7.03(\mathrm{~m}, 1 \mathrm{H}), 7.06-6.98(\mathrm{~m}, 1 \mathrm{H})$, $6.87-6.68(\mathrm{~m}, 2 \mathrm{H}), 3.61(\mathrm{td}, J=10.3,4.4 \mathrm{~Hz}, 1 \mathrm{H}), 2.70(\mathrm{dd}, J=16.2,5.1 \mathrm{~Hz}, 1 \mathrm{H}), 2.48(\mathrm{dd}, \mathrm{J}=16.3,11.8 \mathrm{~Hz}$, $1 \mathrm{H}), 2.22-2.15(\mathrm{~m}, 1 \mathrm{H}), 1.99-1.92(\mathrm{~m}, 1 \mathrm{H}), 1.92-1.84(\mathrm{~m}, 1 \mathrm{H}), 1.77-1.60(\mathrm{~m}, 2 \mathrm{H}), 1.54-1.30(\mathrm{~m}, 3 \mathrm{H})$, 1.07 (tdd, $J=12.9,11.6,3.7 \mathrm{~Hz}, 1 \mathrm{H})$.

${ }^{13}$ C NMR (101 MHz, CDCl $)$ : $\delta .154 .8,129.4,127.1,122.4,119.9,116.3,79.1,37.0,32.5,32.3,31.8,25.4$, 24.6.

MP $77-78^{\circ} \mathrm{C}$

6,6-dimethyl-6H-benzo[c]chromene, ether 3s<smiles>CC1(C)Oc2ccccc2-c2ccccc21</smiles>

Following general method D, ether $3 \mathrm{~s}$ was obtained as colourless oil in quantitative yield $(0.5 \mathrm{mmol}, 105 \mathrm{mg})$. ${ }^{1} \mathbf{H}$ NMR (400 MHz, CDCl 3$): \delta 7.74(\mathrm{dt}, J=7.3,1.6 \mathrm{~Hz}, 2 \mathrm{H}), 7.40-7.19(\mathrm{~m}, 4 \mathrm{H}), 7.03(\mathrm{td}, J=7.5,1.2 \mathrm{~Hz}$, $1 \mathrm{H}), 6.97(\mathrm{dd}, J=8.0,1.2 \mathrm{~Hz}, 1 \mathrm{H}), 1.65(\mathrm{~s}, 6 \mathrm{H})$.

${ }^{13}$ C NMR (101 MHz, CDCl $): \delta 152.9,139.6,129.5,128.7,128.1,127.8,123.3,123.0,122.6,122.3,121.6$, 118.2, 77.6, 27.7 .

HRMS (ESI): Exact mass calculated for $[\mathrm{M}+\mathrm{H}]^{+}\left(\mathrm{C}_{15} \mathrm{H}_{15} \mathrm{O}\right) \mathrm{m} / \mathrm{z} 211.1114$, found 211.1117.

$\underline{\text { 3,3-dimethyl-2,3-dihydro-1H-benzo[f]chromene, ether } \mathbf{3 t} \text { and } \mathbf{3 t}^{\mathbf{3 4}}}$<smiles>CC1(C)CCc2ccc3ccccc3c2O1</smiles>

Following general method D, ether $\mathbf{3 t}$ was obtained as colorless oil in $49 \%$ yield $(0.25 \mathrm{mmol}, 52 \mathrm{mg})$.

${ }^{1}$ H NMR (400 MHz, CDCl $\left.\mathbf{3}\right): \delta 8.27-8.17(\mathrm{~m}, 1 \mathrm{H}), 7.79-7.72(\mathrm{~m}, 1 \mathrm{H}), 7.53-7.38(\mathrm{~m}, 2 \mathrm{H}), 7.33(\mathrm{~d}, J=8.3$ $\mathrm{Hz}, 1 \mathrm{H}), 7.18(\mathrm{~d}, J=8.3 \mathrm{~Hz}, 1 \mathrm{H}), 2.90(\mathrm{t}, J=6.7 \mathrm{~Hz}, 2 \mathrm{H}), 1.92(\mathrm{t}, J=6.7 \mathrm{~Hz}, 2 \mathrm{H}), 1.45(\mathrm{~s}, 6 \mathrm{H})$.

${ }^{13}$ C NMR (101 MHz, CDCl $): \delta$ 148.7, 133.3, 127.7, 127.3, 125.7, 125.5, 124.9, 121.6, 118.8, 114.3, 74.5, 32.8, $26.9,22.8$.<smiles>CC1(C)CCc2c(ccc3ccccc23)O1</smiles>

Following general method D, ether 3t' was obtained as white solid in $11 \%$ yield $(0.06 \mathrm{mmol}, 12 \mathrm{mg})$.

${ }^{1} \mathrm{H}$ NMR $\left(400 \mathrm{MHz}, \mathbf{C D C l}_{3}\right): \delta 7.83(\mathrm{~d}, J=8.5 \mathrm{~Hz}, 1 \mathrm{H}), 7.76(\mathrm{~d}, J=8.1 \mathrm{~Hz}, 1 \mathrm{H}), 7.62(\mathrm{~d}, J=9.0 \mathrm{~Hz}, 1 \mathrm{H}), 7.48$ $(\mathrm{t}, J=7.6 \mathrm{~Hz}, 1 \mathrm{H}), 7.33(\mathrm{t}, J=7.5 \mathrm{~Hz}, 1 \mathrm{H}), 7.03(\mathrm{~d}, J=8.9 \mathrm{~Hz}, 1 \mathrm{H}), 3.04(\mathrm{t}, J=6.9 \mathrm{~Hz}, 2 \mathrm{H}), 1.96(\mathrm{t}, J=6.8 \mathrm{~Hz}$, $2 \mathrm{H}), 1.39(\mathrm{~s}, 6 \mathrm{H})$.

${ }^{13} \mathbf{C}$ NMR (101 MHz, $\left.\mathbf{C D C l}_{3}\right): \delta 151.3,133.0,128.7,128.3,127.7,126.1,122.9,121.8,119.7,112.4,74.0,32.7$, 26.5, 19.3.

\section{2,2-diphenylchromane, ether $\mathbf{3 u}$}<smiles>c1ccc(C2(c3ccccc3)CCc3ccccc3O2)cc1</smiles>

Following general method D, ether $3 \mathbf{u}$ was obtained as colourless oil in $54 \%$ yield $(0.27 \mathrm{mmol}, 77 \mathrm{mg})$.

\footnotetext{
${ }^{33}$ Antus, S.; Baitz-Gacs, E.; Snatzke, G.; Vas, J. Tetrahedron 1986, $42,5637$.

${ }^{34}$ Youn, S. W.; Eom, J. I. J. Org. Chem. 2006, 71, 6705.
} 


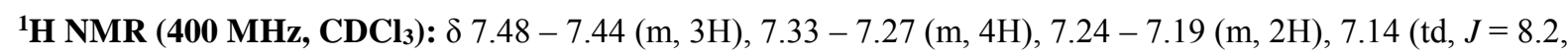
7.3, 1.6 Hz, 1H), $7.07(\mathrm{dd}, J=8.2,1.4 \mathrm{~Hz}, 1 \mathrm{H}), 6.95(\mathrm{dd}, J=7.4,1.6 \mathrm{~Hz}, 1 \mathrm{H}), 6.81(\mathrm{td}, J=7.3,1.4 \mathrm{~Hz}, 1 \mathrm{H})$, $2.73-2.61(\mathrm{~m}, 4 \mathrm{H})$.

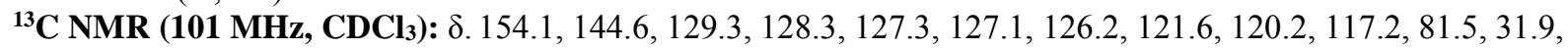
22.8 .

HRMS (ESI): Exact mass calculated for $[\mathrm{M}+\mathrm{H}]^{+}\left(\mathrm{C}_{21} \mathrm{H}_{19} \mathrm{O}\right) \mathrm{m} / \mathrm{z} 287.1437$, found 287.1430. 
7. NMR SPECTRUMS

$\underline{\mathbf{1 k}},{ }^{1} \mathrm{H}$ NMR (400 MHz, $\left.\mathrm{CDCl}_{3}\right)$

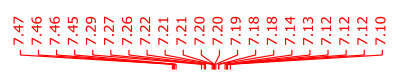
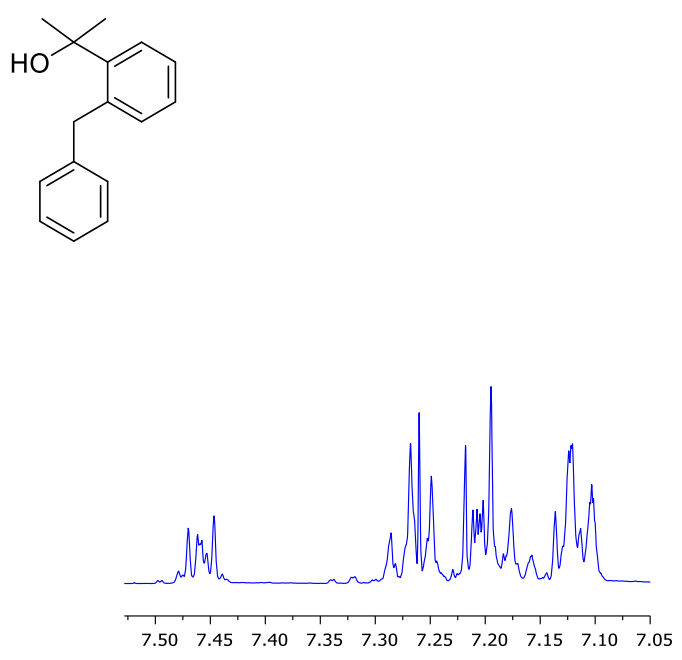

$\stackrel{\substack{0 \\ ⿱ 亠 乂}}{i}$

$\stackrel{\leftrightarrow}{i}$
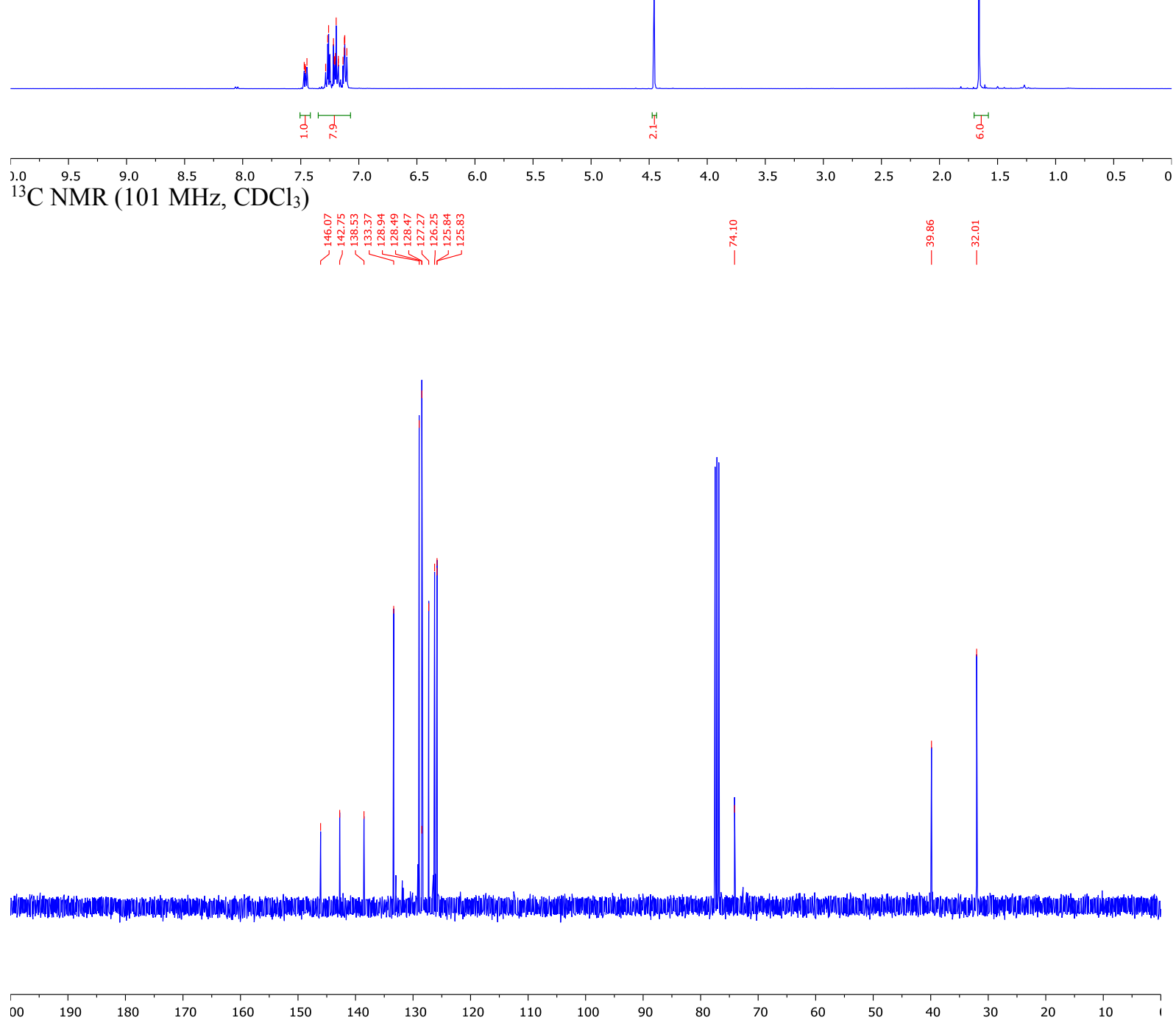

S23 
1t, ${ }^{1} \mathrm{H}$ NMR (400 MHz, $\left.\mathrm{CDCl}_{3}\right)$

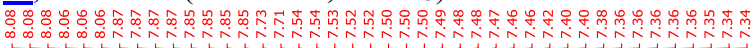

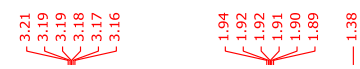

(1)
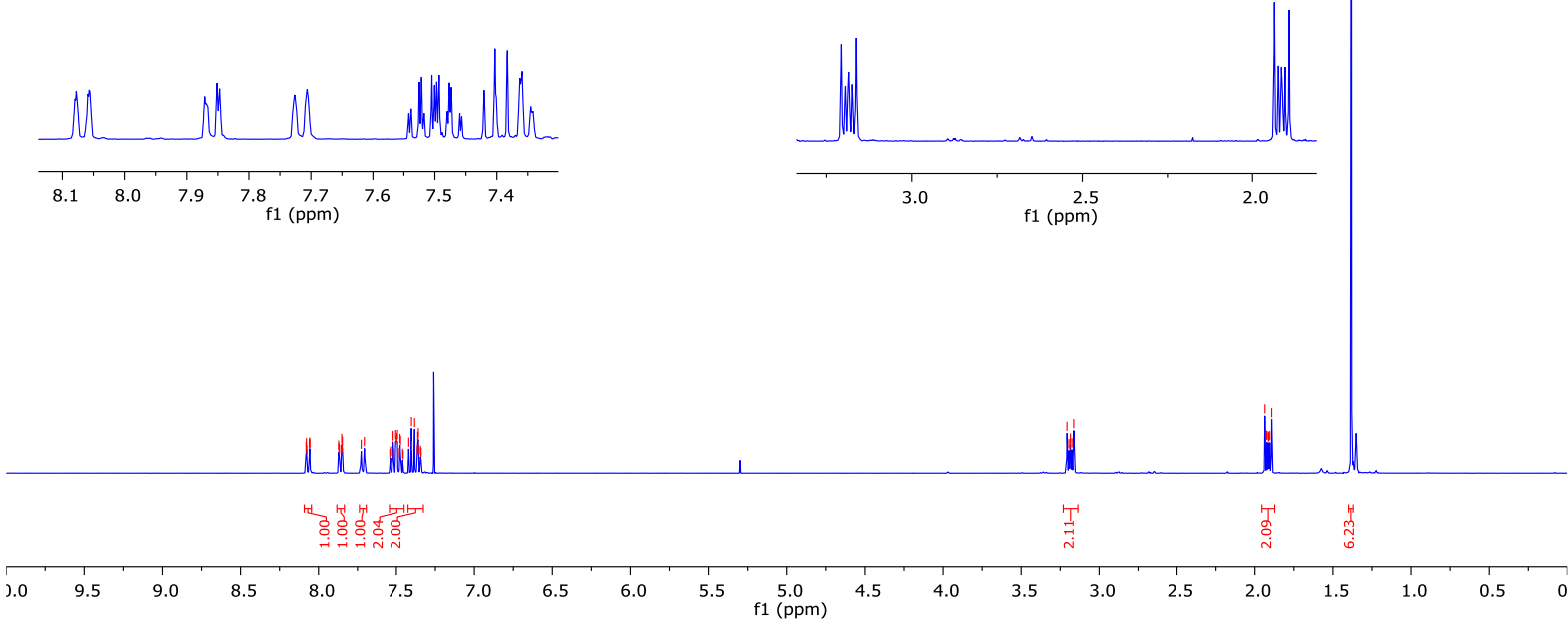

${ }^{13} \mathrm{C}$ NMR (101 MHz, $\left.\mathrm{CDCl}_{3}\right)$

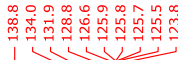

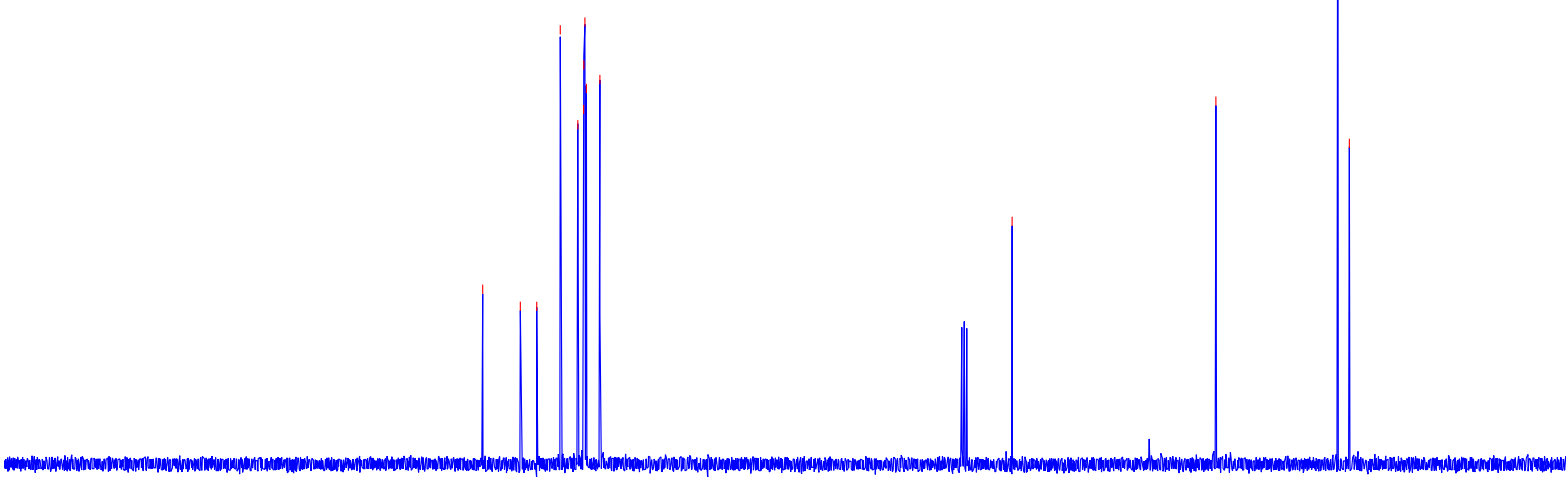

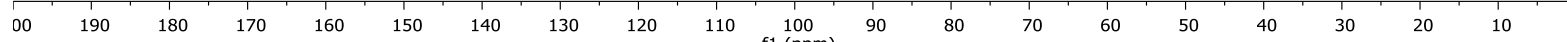


2a, ${ }^{1} \mathrm{H}$ NMR $\left(400 \mathrm{MHz}, \mathrm{CDCl}_{3}\right)$

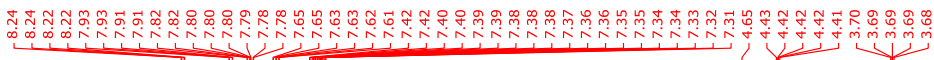
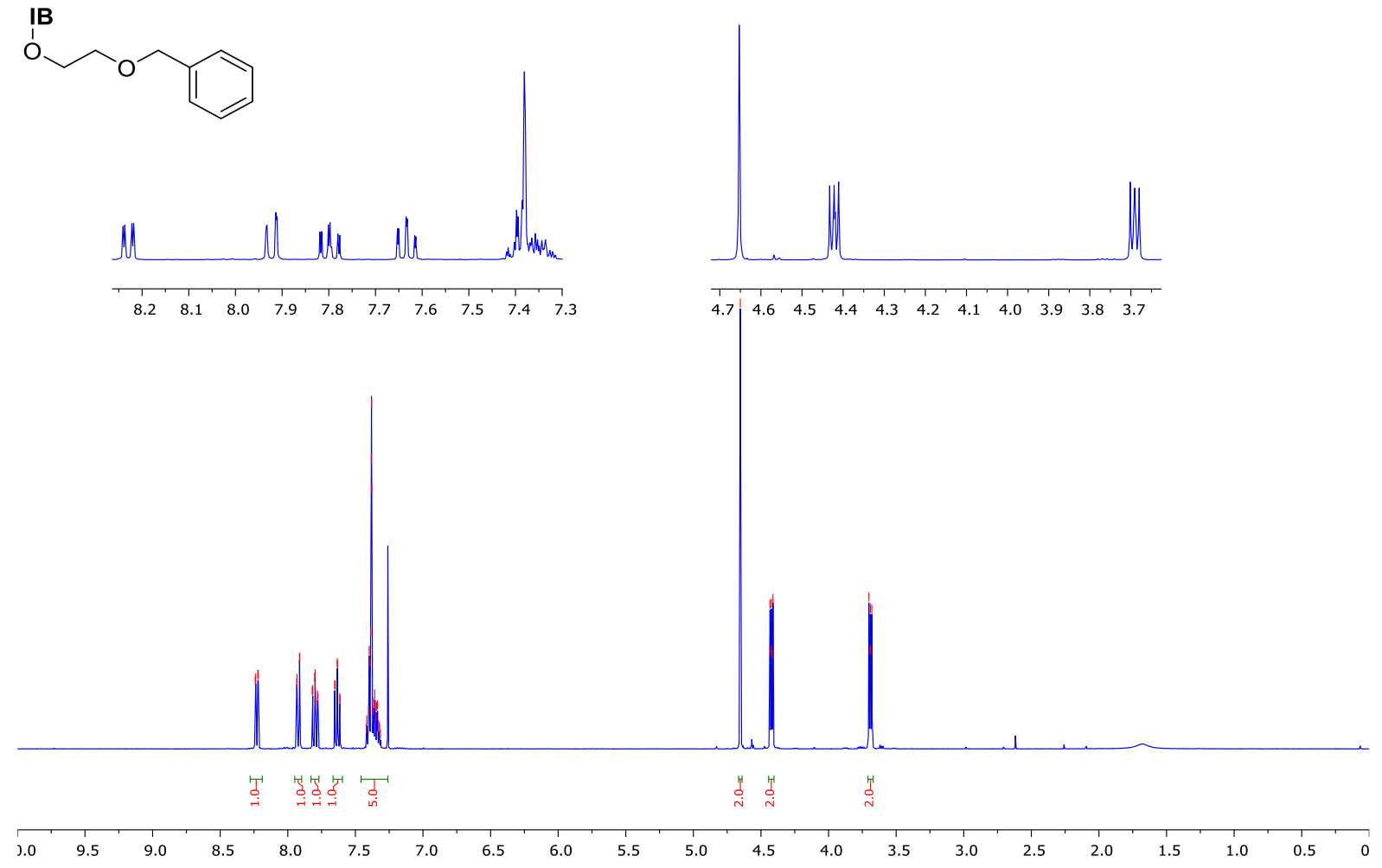

${ }^{13} \mathrm{C}$ NMR (101 MHz, $\left.\mathrm{CDCl}_{3}\right)$
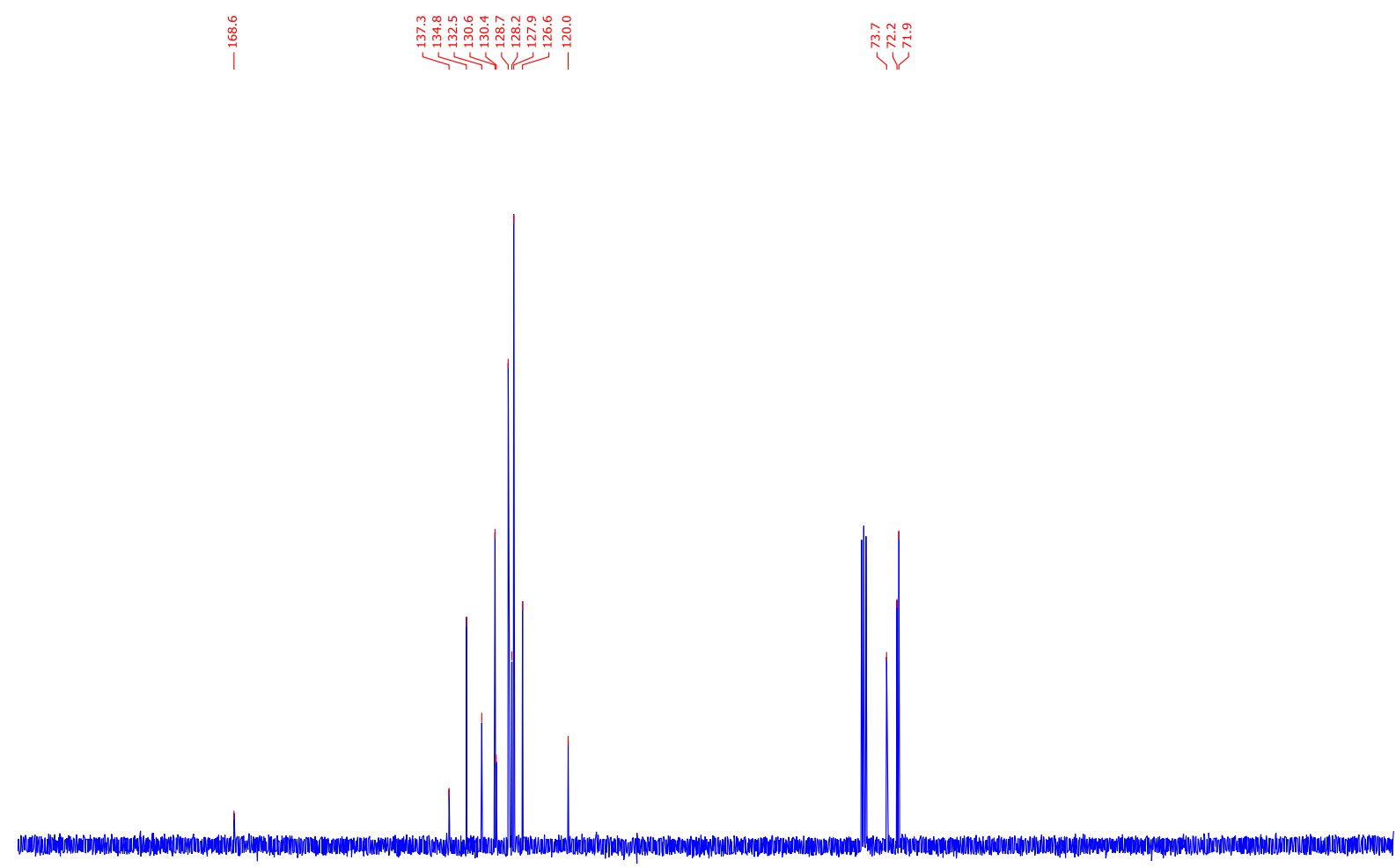

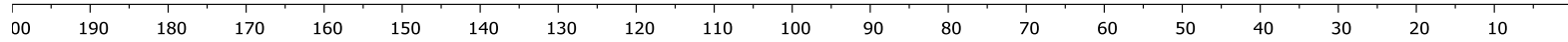


$\underline{\text { Lb }},{ }^{1} \mathrm{H}$ NMR (400 MHz, $\left.\mathrm{CDCl}_{3}\right)$

ب

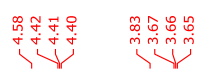
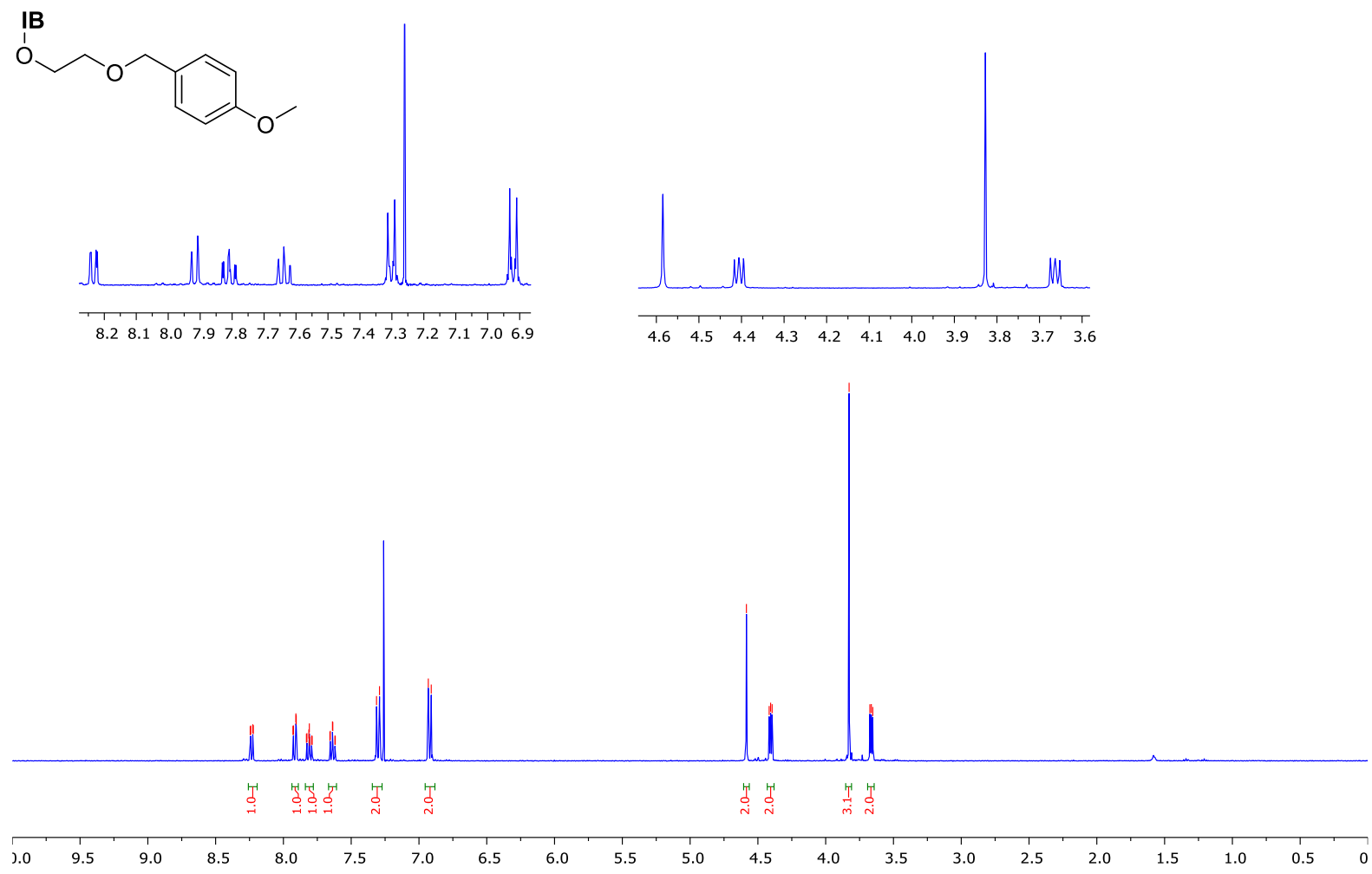

${ }^{13} \mathrm{C}$ NMR (101 MHz, $\mathrm{CDCl}_{3}$ )
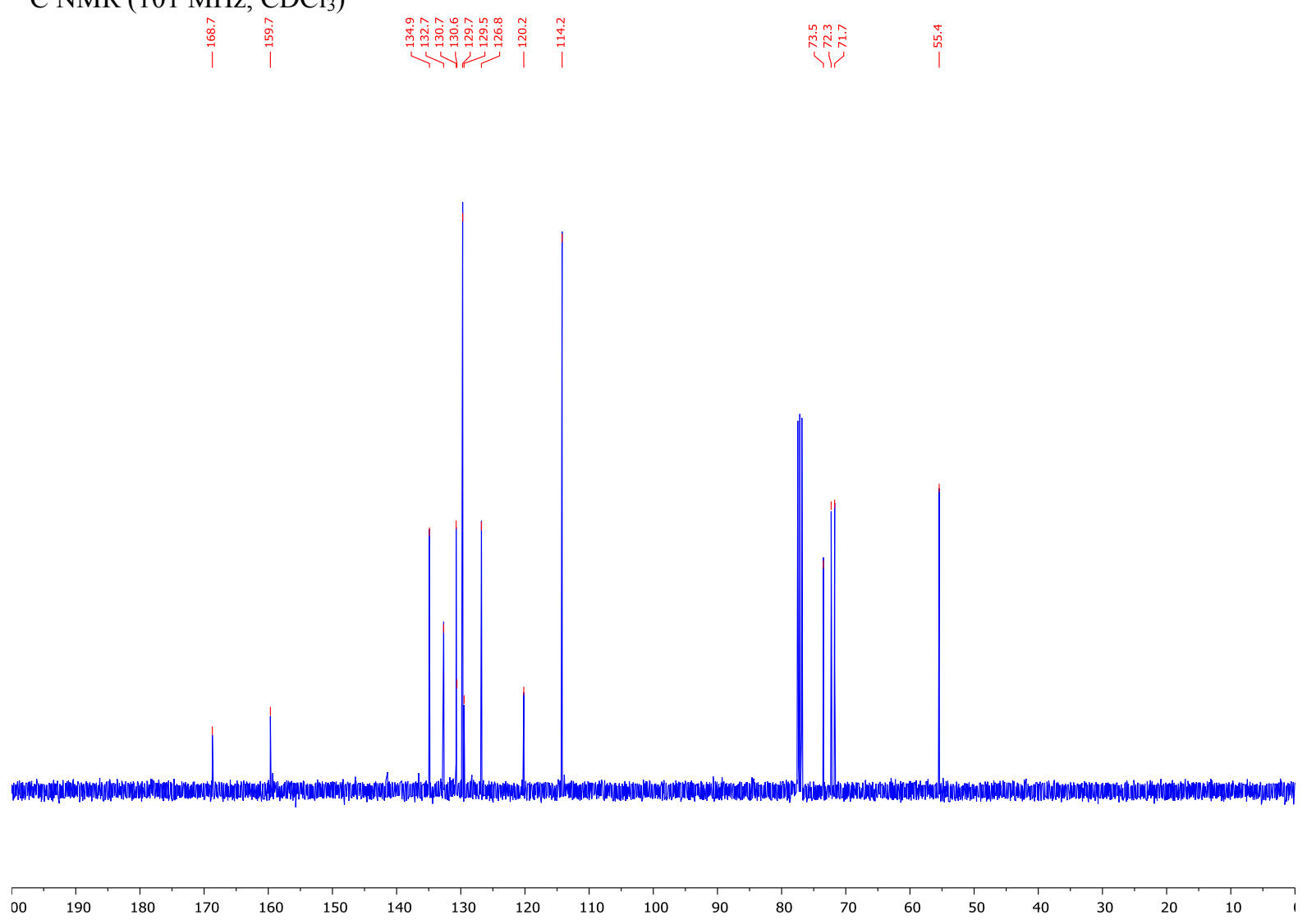

S26 
2c, ${ }^{1} \mathrm{H}$ NMR (400 MHz, $\left.\mathrm{CDCl}_{3}\right)$

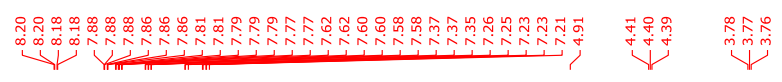<smiles>[18F]CCOCc1c(Cl)cccc1Cl</smiles>
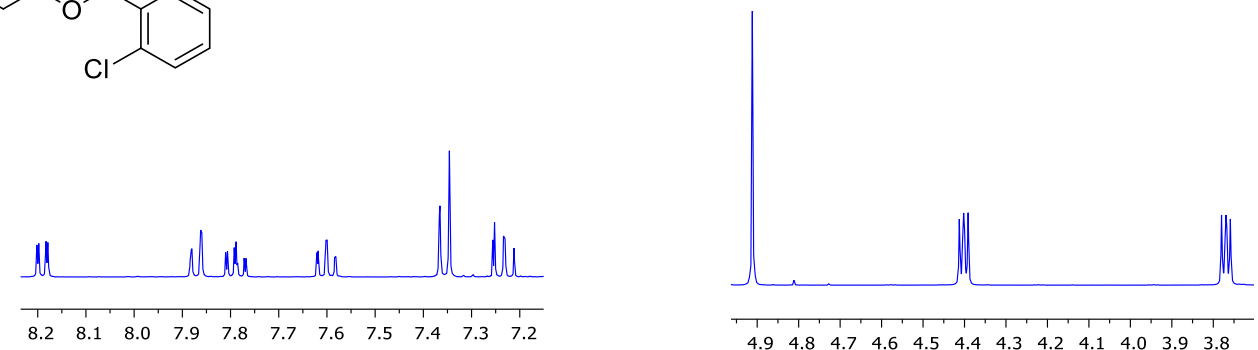

$\begin{array}{llllllllllllllllll}4.9 & 4.8 & 4.7 & 4.6 & 4.5 & 4.4 & 4.3 & 4.2 & 4.1 & 4.0 & 3.9 & 3.8\end{array}$

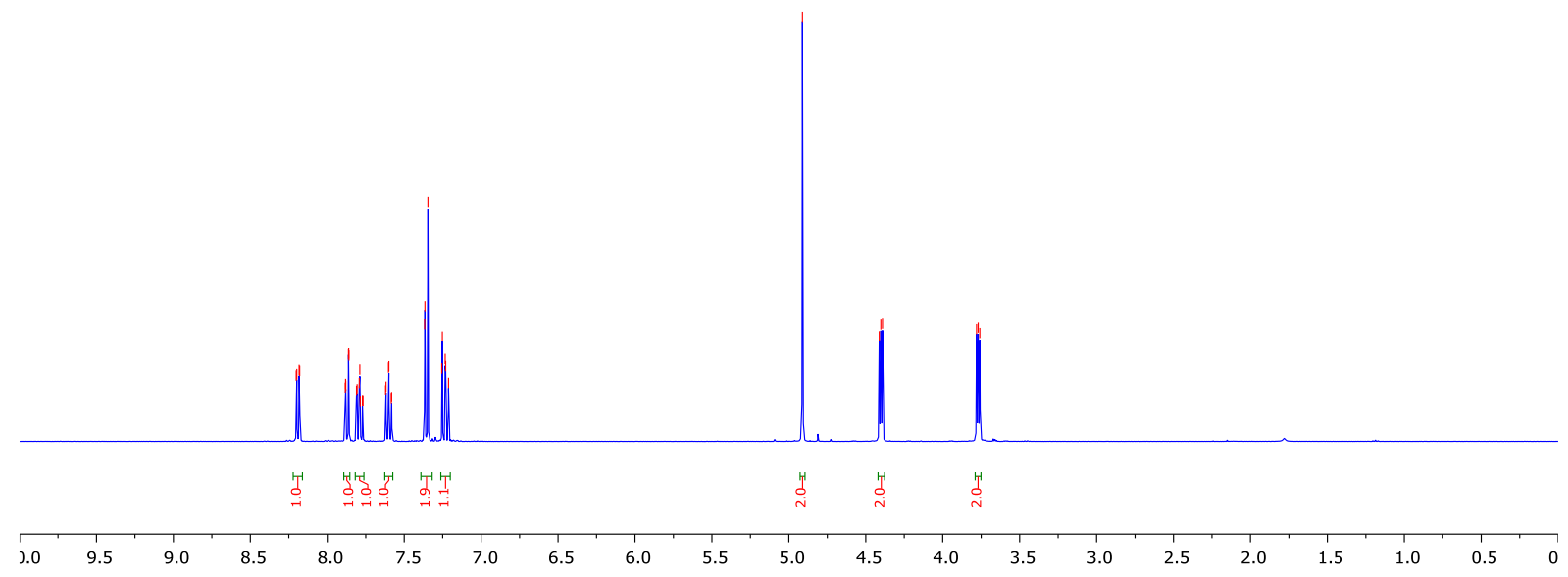

${ }^{13} \mathrm{C}$ NMR $\left(101 \mathrm{MHz}, \mathrm{CDCl}_{3}\right)$
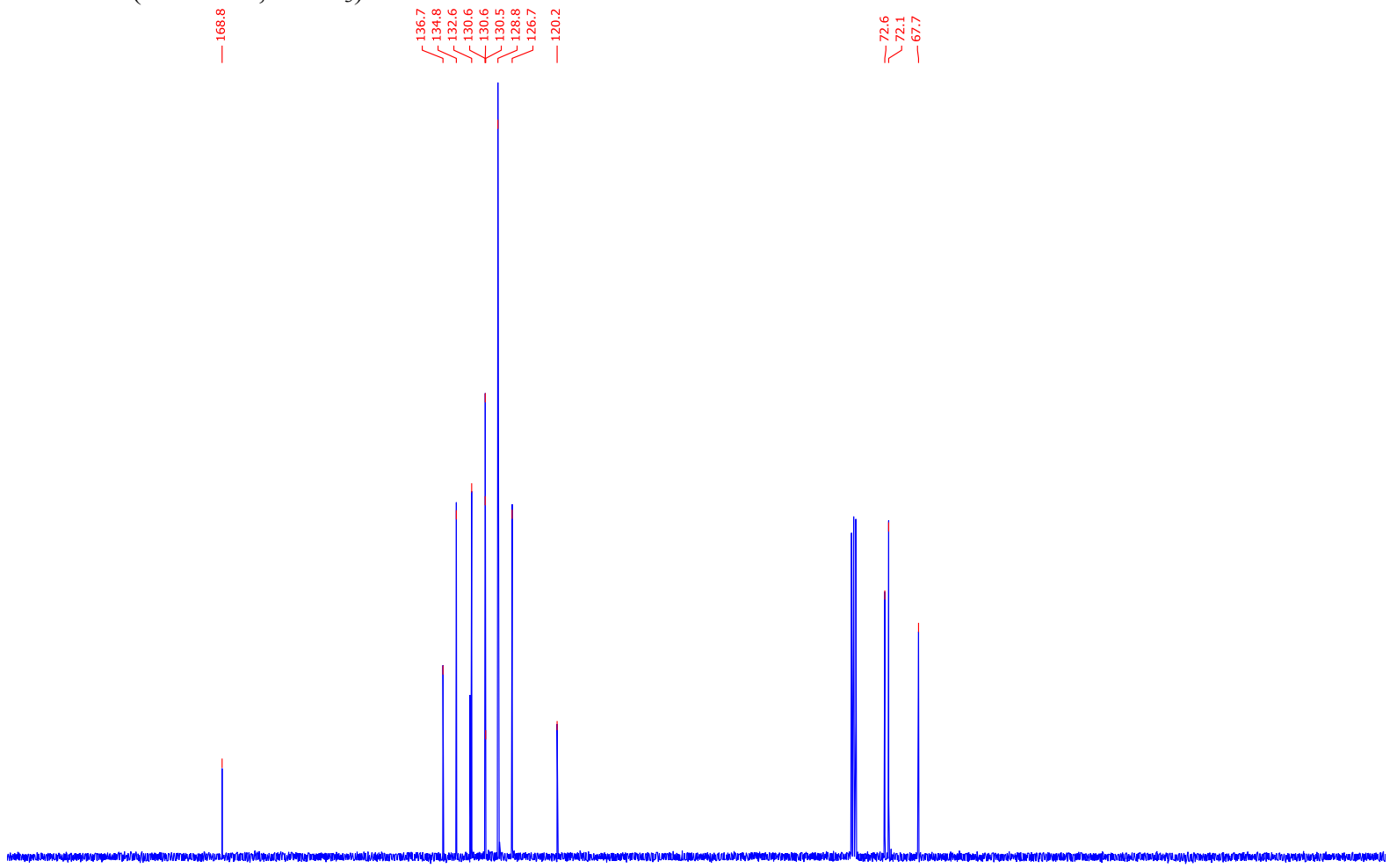

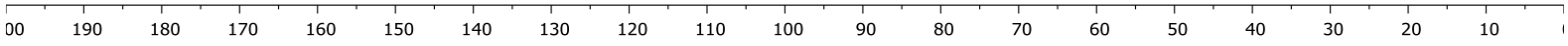


$\underline{\text { 2e }},{ }^{1} \mathrm{H} \mathrm{NMR}\left(400 \mathrm{MHz}, \mathrm{CDCl}_{3}\right)$

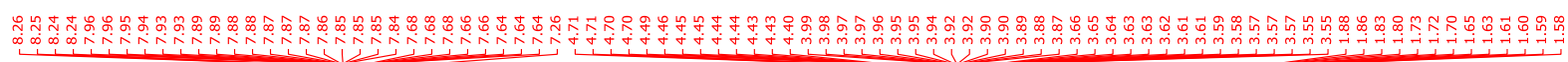
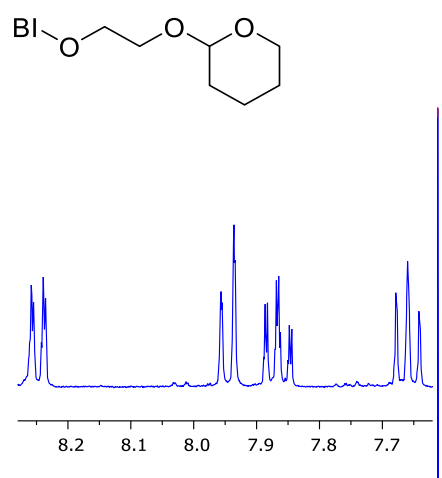

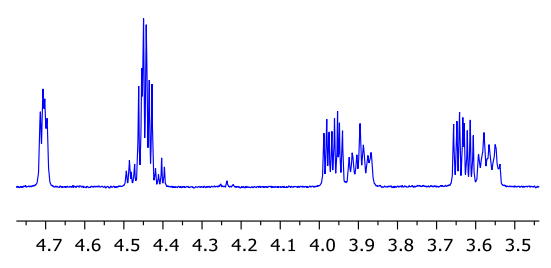

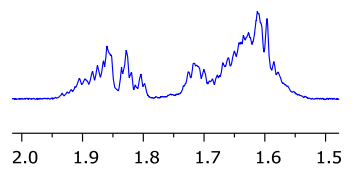

${ }^{13} \mathrm{C}$ NMR (101 MHz, $\left.\mathrm{CDCl}_{3}\right)$

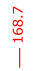

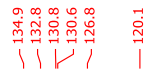

$\stackrel{\circ}{\circ}$

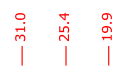

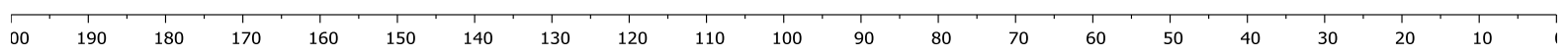

S29 
2f., ${ }^{1} \mathrm{H}$ NMR (400 MHz, $\left.\mathrm{CDCl}_{3}\right)$

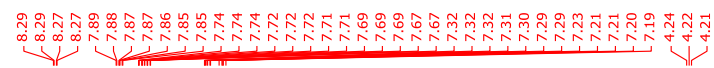

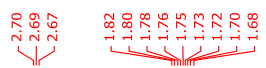

IB<smiles>[O]CCCCc1ccccc1</smiles>

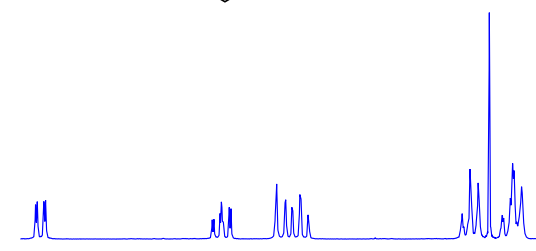

$\begin{array}{lllllllllllllllll}8.3 & 8.2 & 8.1 & 8.0 & 7.9 & 7.8 & 7.7 & 7.6 & 7.5 & 7.4 & 7.3 & 7.2\end{array}$
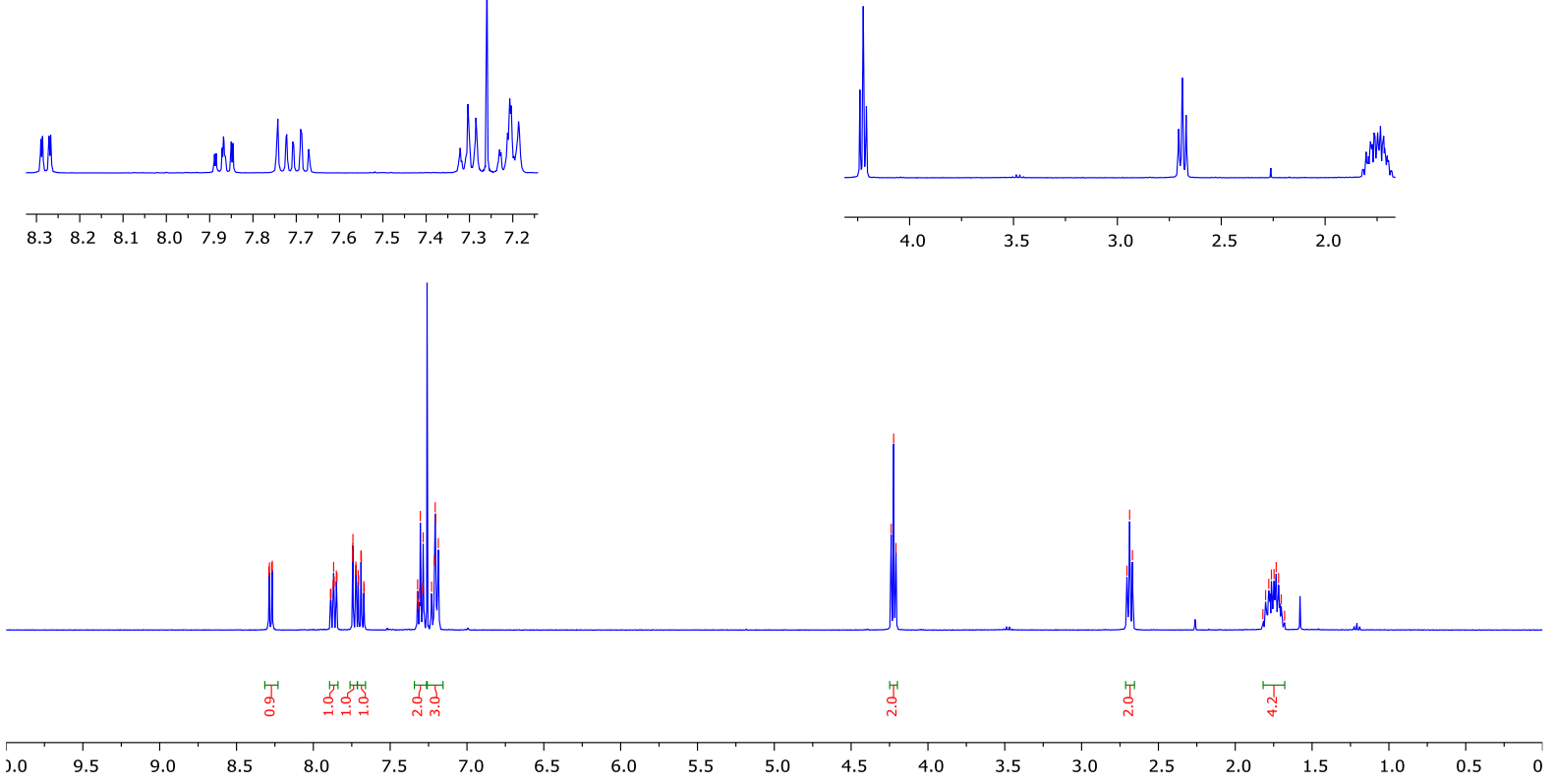

${ }^{13} \mathrm{C}$ NMR (101 MHz, $\left.\mathrm{CDCl}_{3}\right)$

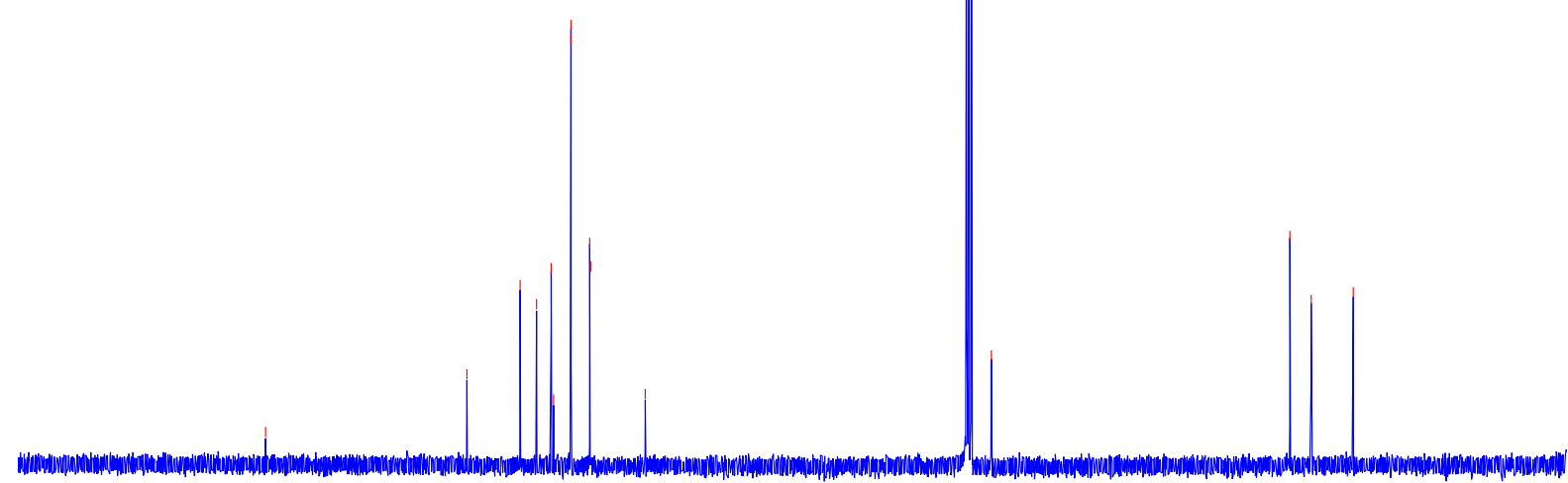

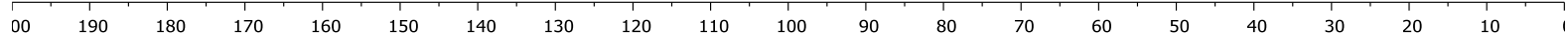


2g, ${ }^{1} \mathrm{H}$ NMR (400 MHz, $\left.\mathrm{CDCl}_{3}\right)$

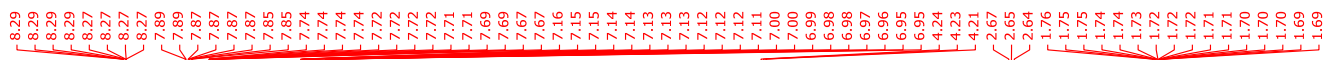

IB<smiles>COCCCCc1ccc(F)cc1</smiles>

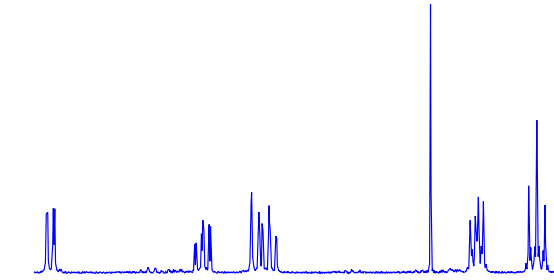

$\begin{array}{lllllllllllllllll}8.3 & 8.2 & 8.1 & 8.0 & 7.9 & 7.8 & 7.7 & 7.6 & 7.5 & 7.4 & 7.3 & 7.2 & 7.1 & 7.0\end{array}$
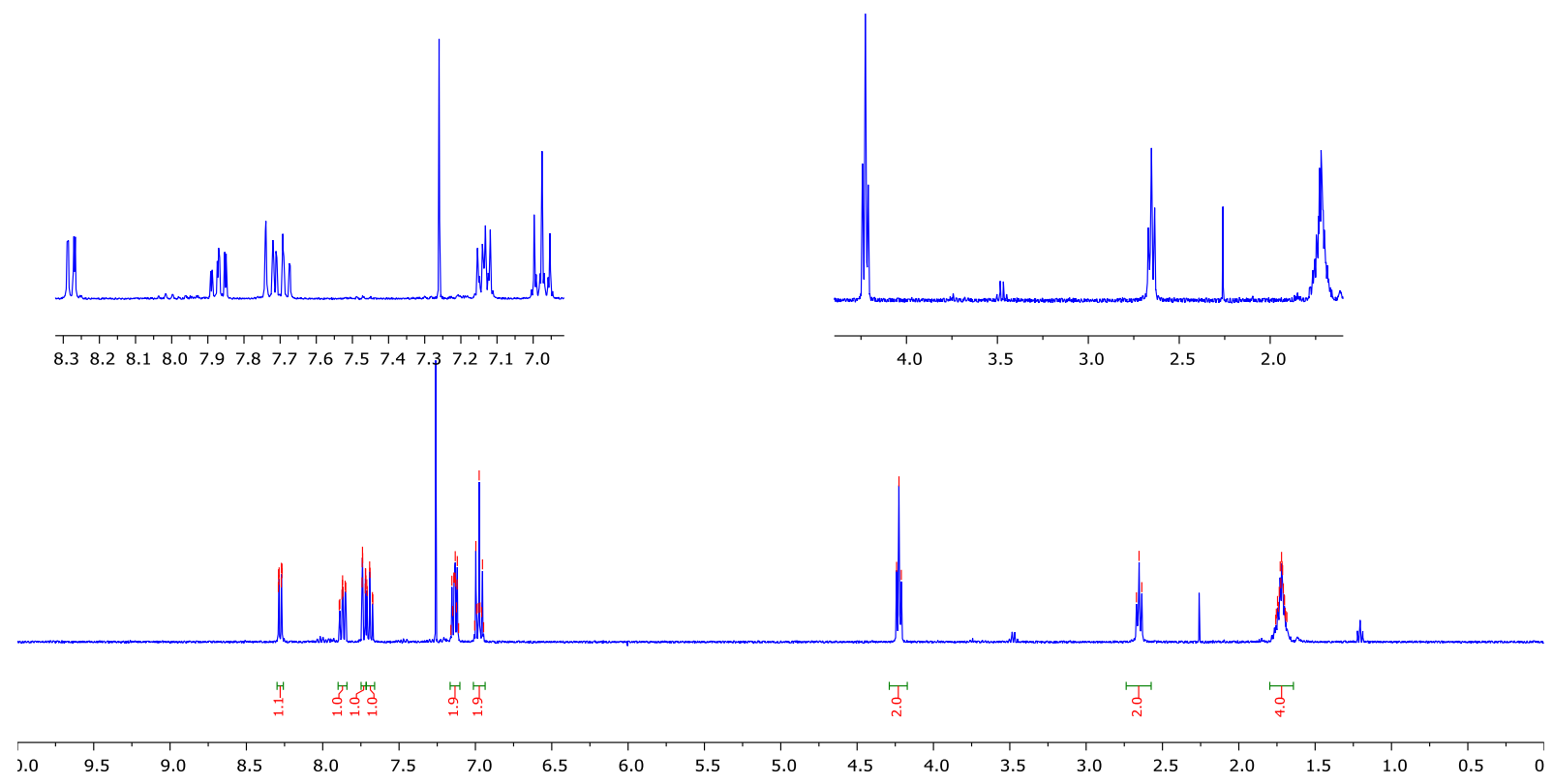

${ }^{13} \mathrm{C}$ NMR (101 MHz, $\left.\mathrm{CDCl}_{3}\right)$

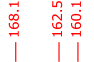

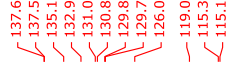
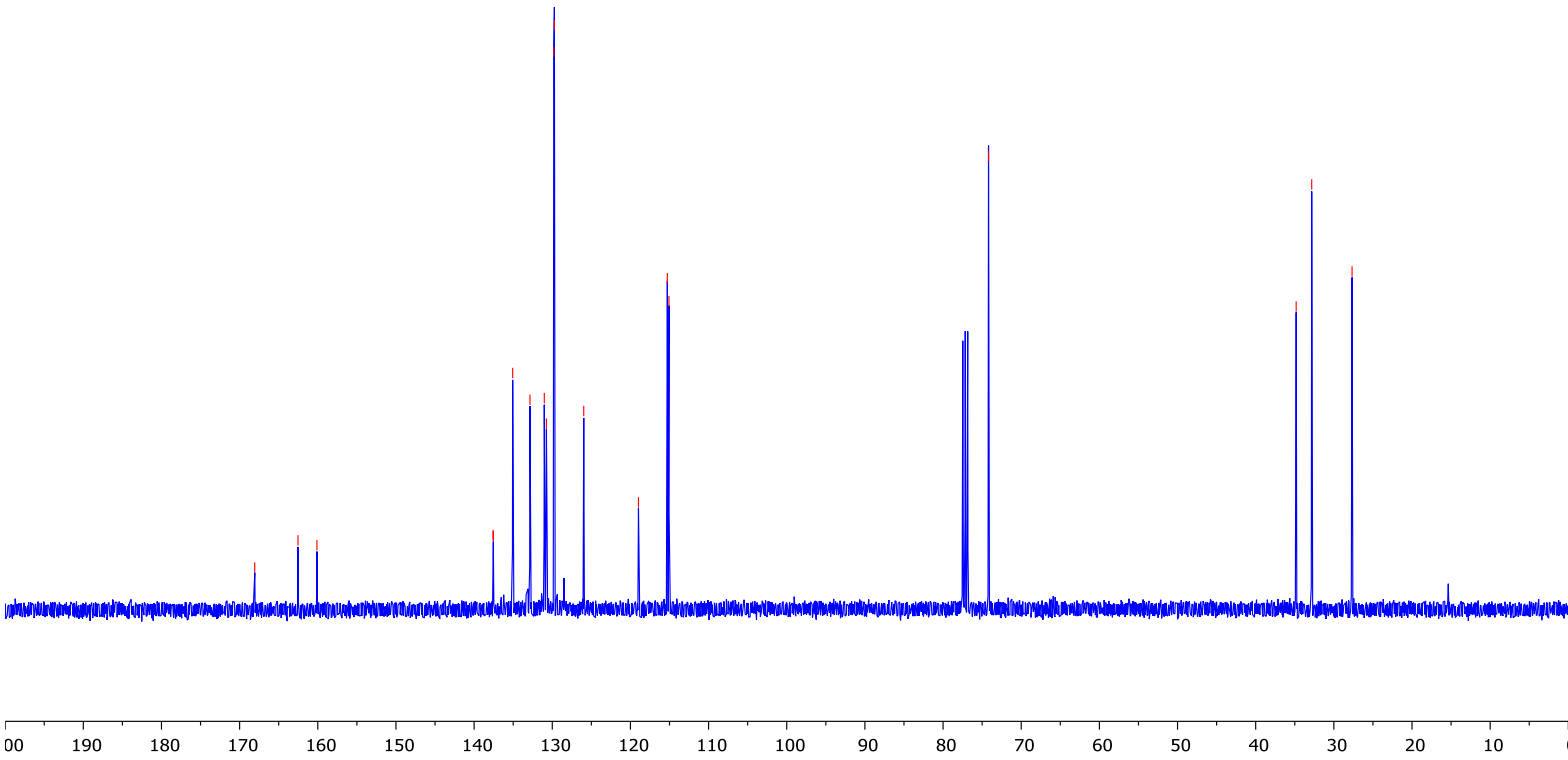
$\underline{\text { 2h. }},{ }^{1} \mathrm{H}$ NMR $\left(400 \mathrm{MHz}, \mathrm{CDCl}_{3}\right)$

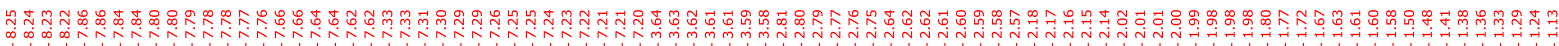<smiles>CCCCCc1ccccc1</smiles>
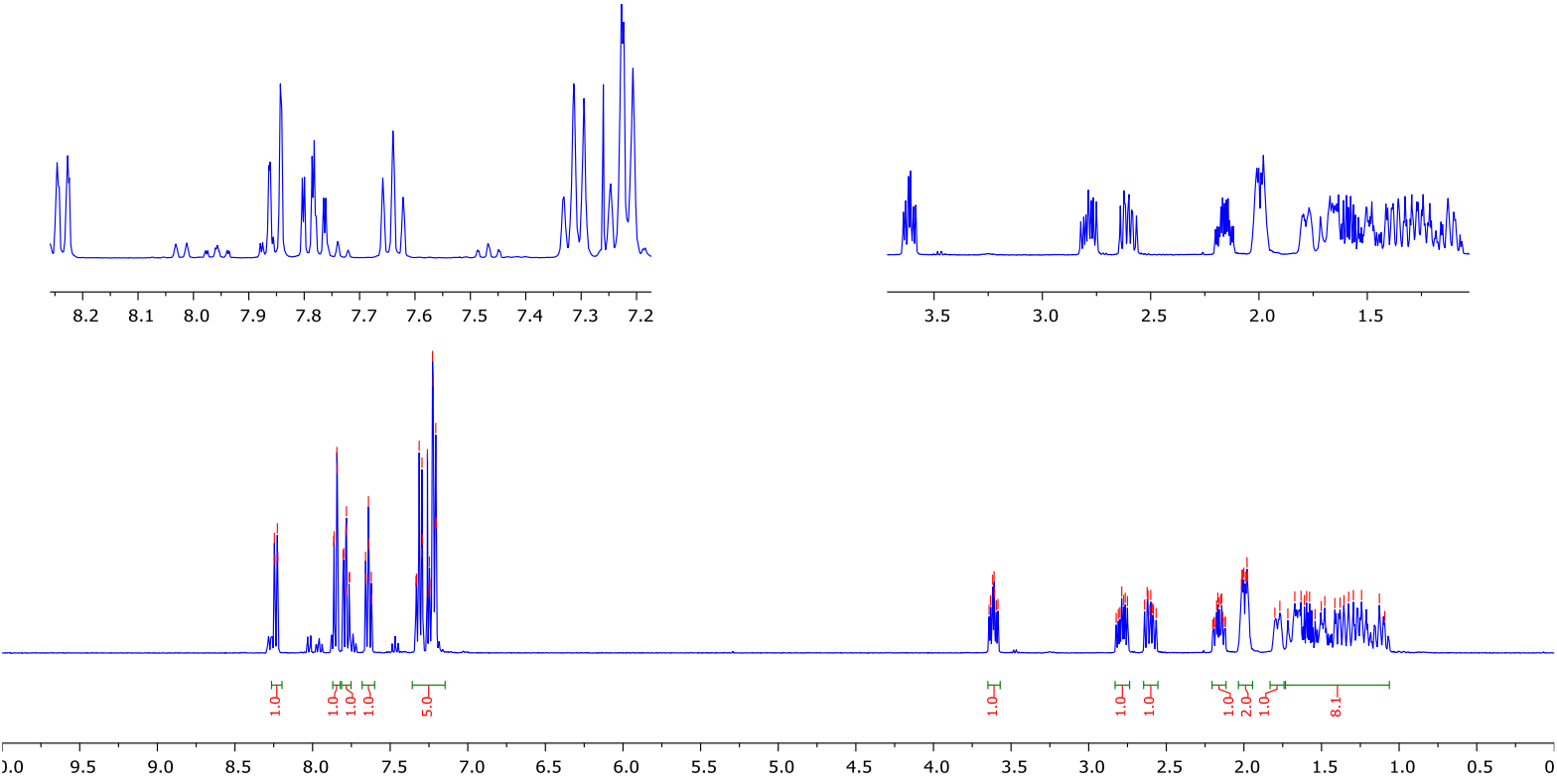

${ }^{13} \mathrm{C}$ NMR (101 MHz, $\mathrm{CDCl}_{3}$ )

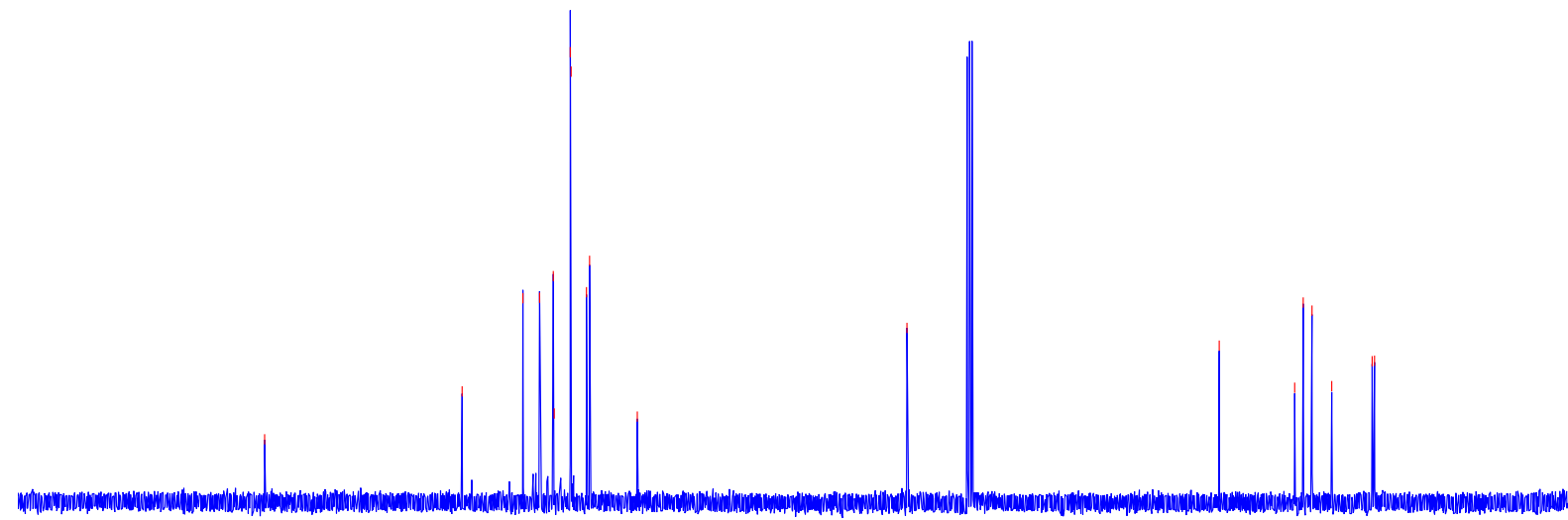

\begin{tabular}{llllllllllllllllllllll}
\hline 00 & 190 & 180 & 170 & 160 & 150 & 140 & 130 & 120 & 110 & 100 & 90 & 80 & 70 & 60 & 50 & 40 & 30 & 20 & 10
\end{tabular} 
$\underline{\text { 2i, }}{ }^{1} \mathrm{H}$ NMR $\left(400 \mathrm{MHz}, \mathrm{CDCl}_{3}\right)$

品

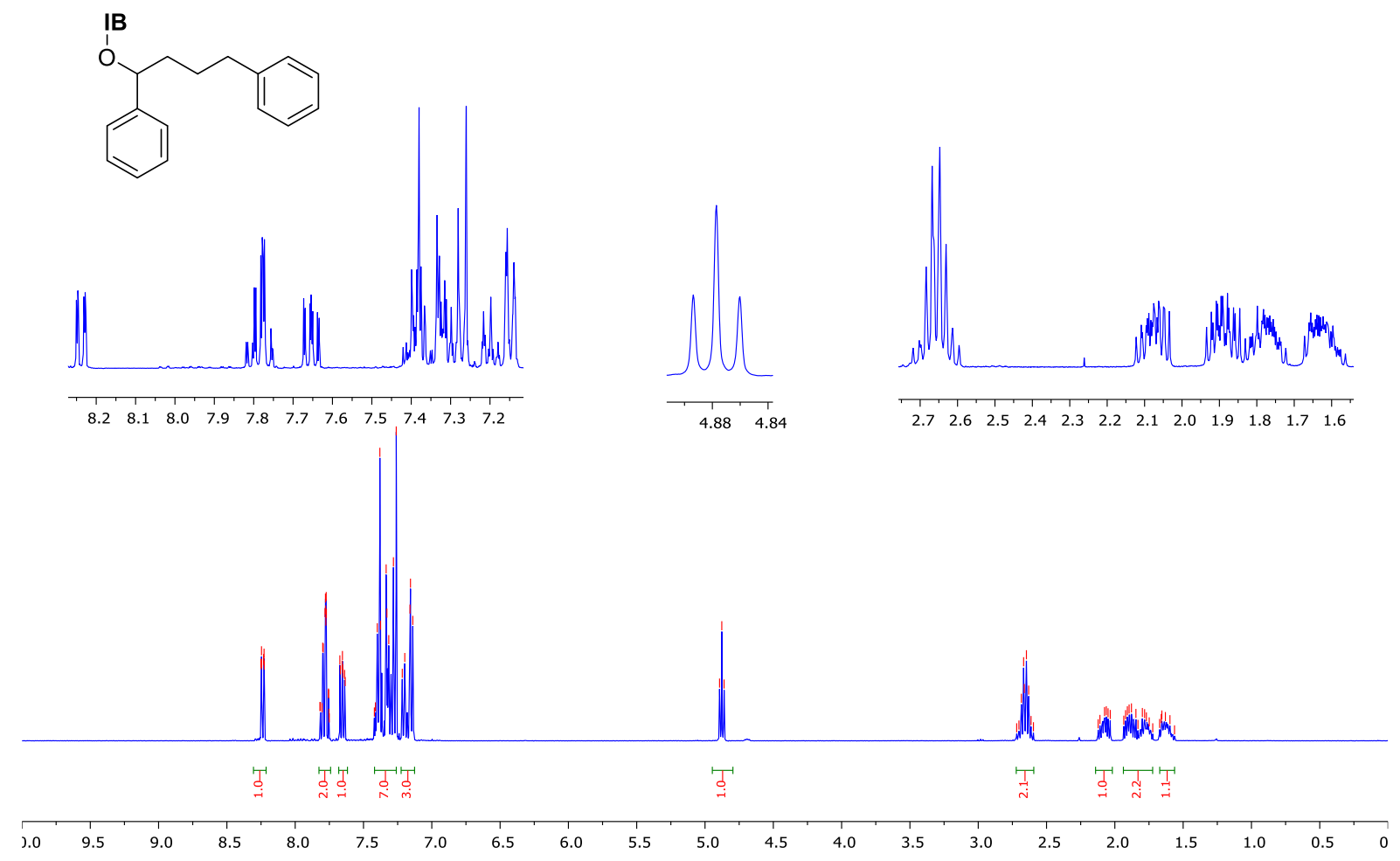

${ }^{13} \mathrm{C} \mathrm{NMR}\left(101 \mathrm{MHz}, \mathrm{CDCl}_{3}\right)$

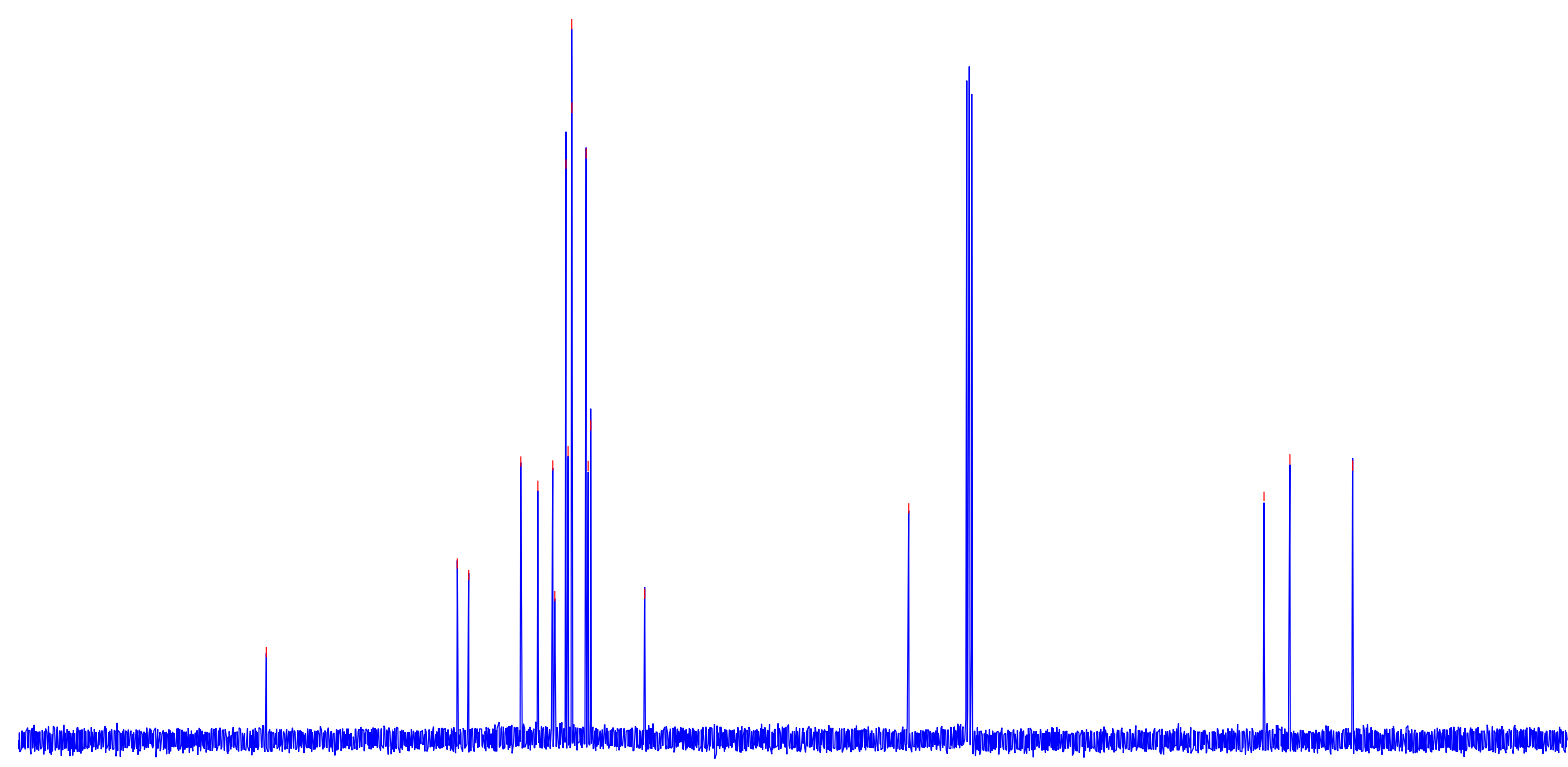

\begin{tabular}{llllllllllllllllllll}
\hline 00 & 190 & 180 & 170 & 160 & 150 & 140 & 130 & 120 & 110 & 100 & 90 & 80 & 70 & 60 & 50 & 40 & 30 & 20 & 10
\end{tabular} 
2j. ${ }^{1} \mathrm{H}$ NMR $\left(400 \mathrm{MHz}, \mathrm{CDCl}_{3}\right)$

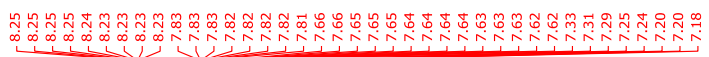

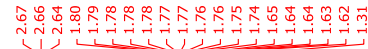<smiles>[B]OC(C)(C)CCCc1ccccc1</smiles>
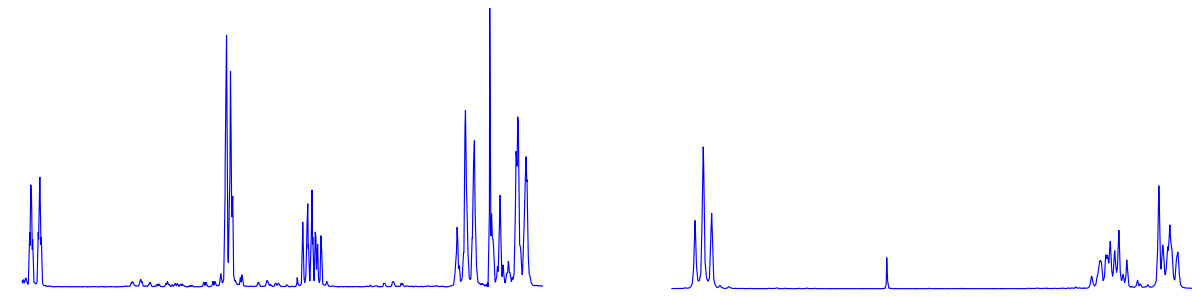

$\begin{array}{lllllllllllll}8.2 & 8.1 & 8.0 & 7.9 & 7.8 & 7.7 & 7.6 & 7.5 & 7.4 & 7.3 & 7.2\end{array}$

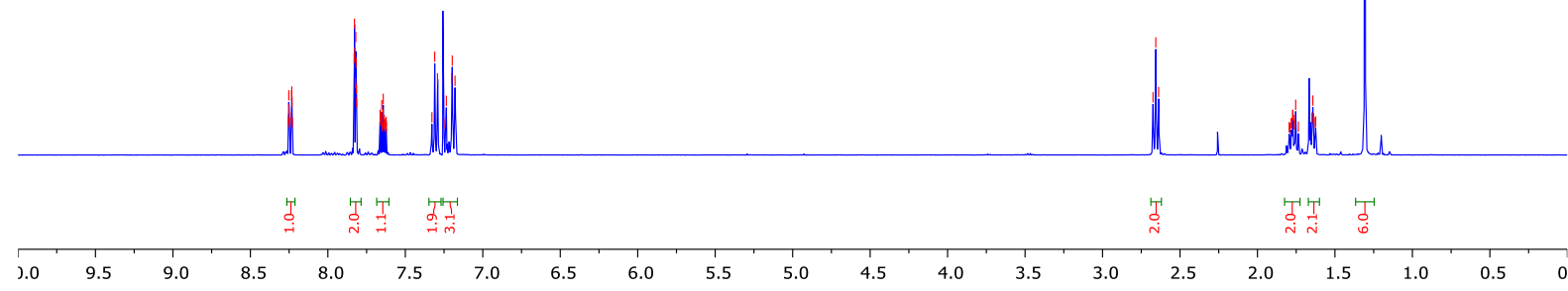

${ }^{13} \mathrm{C}$ NMR (101 MHz, $\left.\mathrm{CDCl}_{3}\right)$

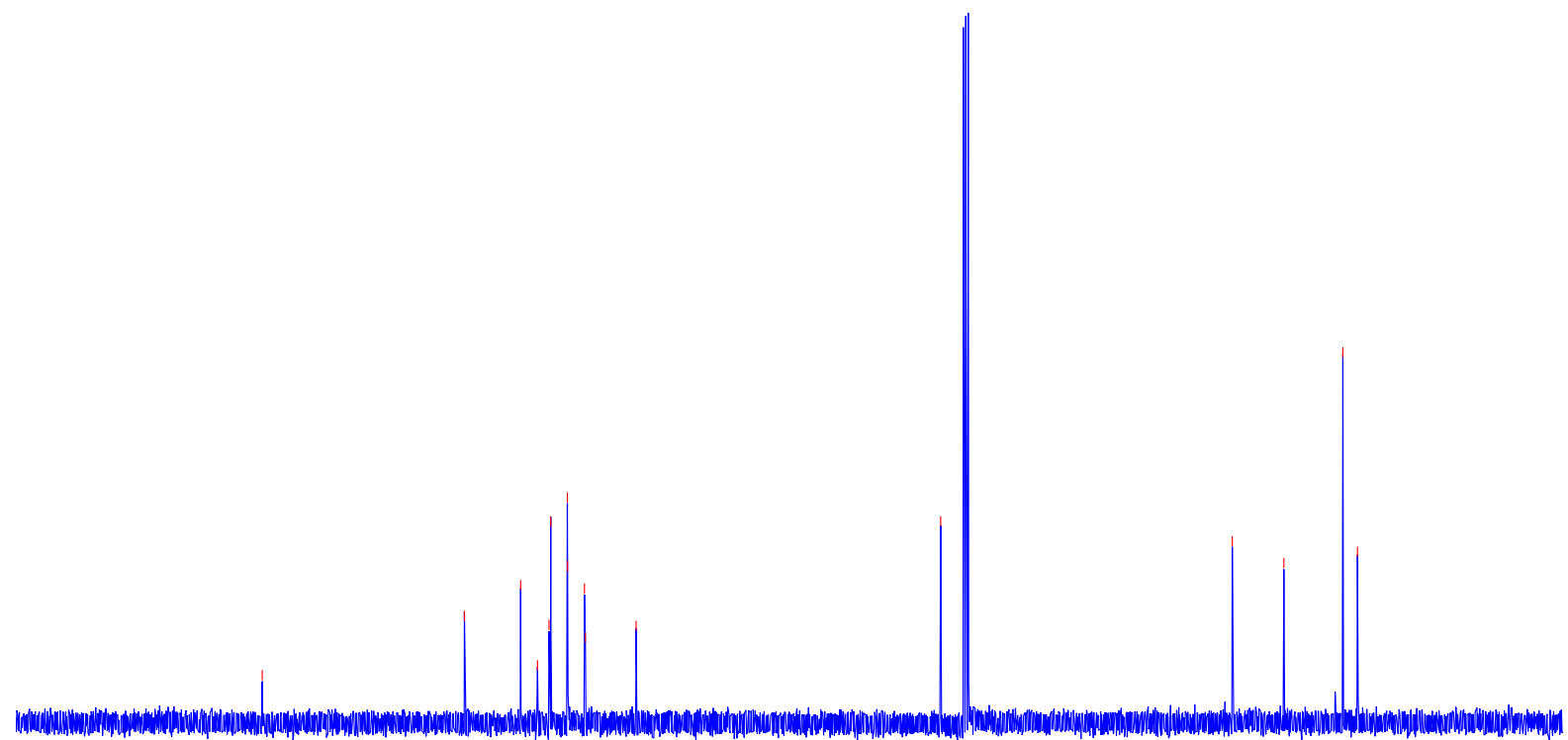

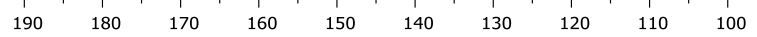

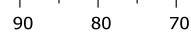


$\underline{\text { 2k }},{ }^{1} \mathrm{H}$ NMR (400 MHz, $\left.\mathrm{CDCl}_{3}\right)$

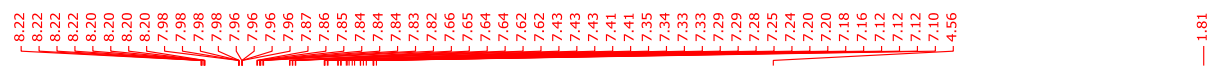<smiles>CC(C)(OBr)c1ccccc1Cc1ccccc1</smiles>

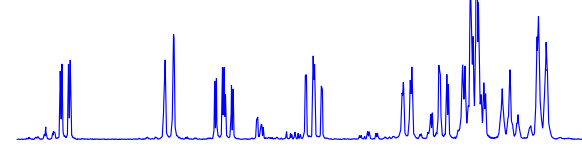

$\begin{array}{lllllllllllllllllll}8.3 & 8.2 & 8.1 & 8.0 & 7.9 & 7.8 & 7.7 & 7.6 & 7.5 & 7.4 & 7.3 & 7.2 & 7.1\end{array}$

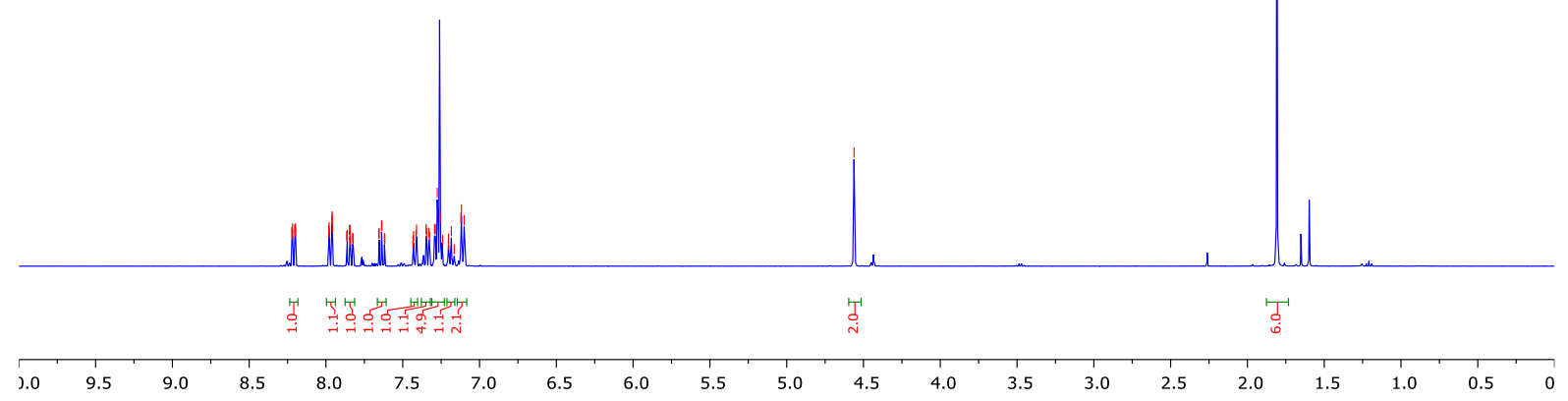

${ }^{13} \mathrm{C}$ NMR (101 MHz, $\left.\mathrm{CDCl}_{3}\right)$

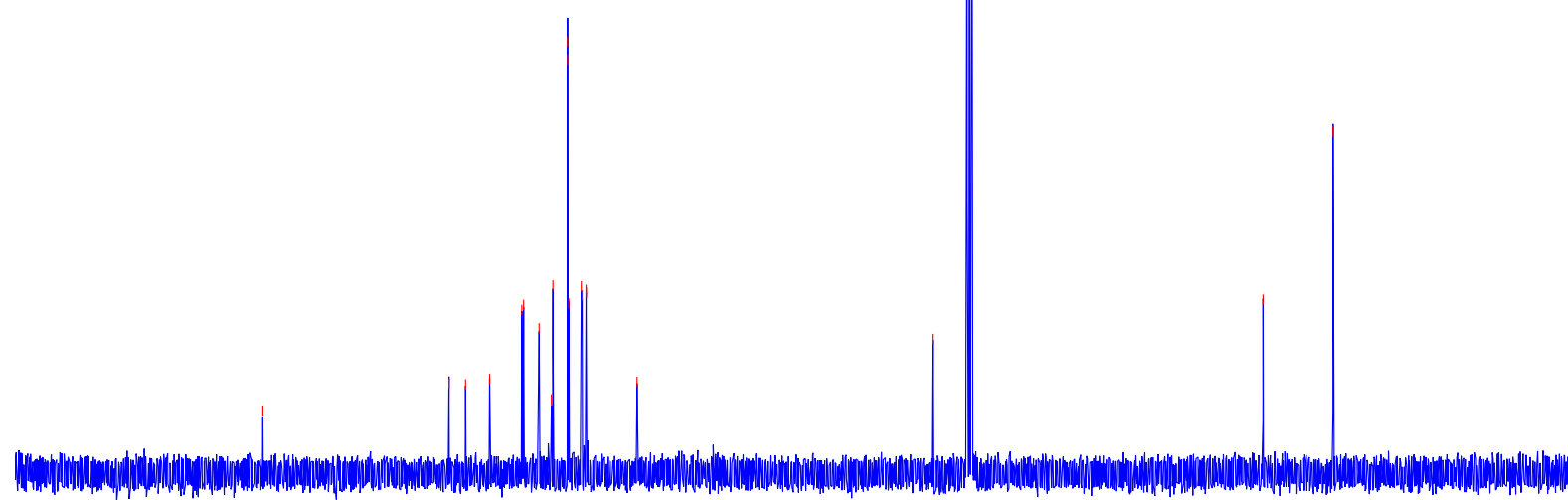

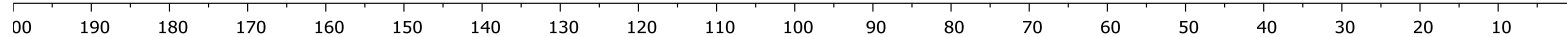


$\underline{21},{ }^{1} \mathrm{H}$ NMR $\left(400 \mathrm{MHz}, \mathrm{CDCl}_{3}\right)$

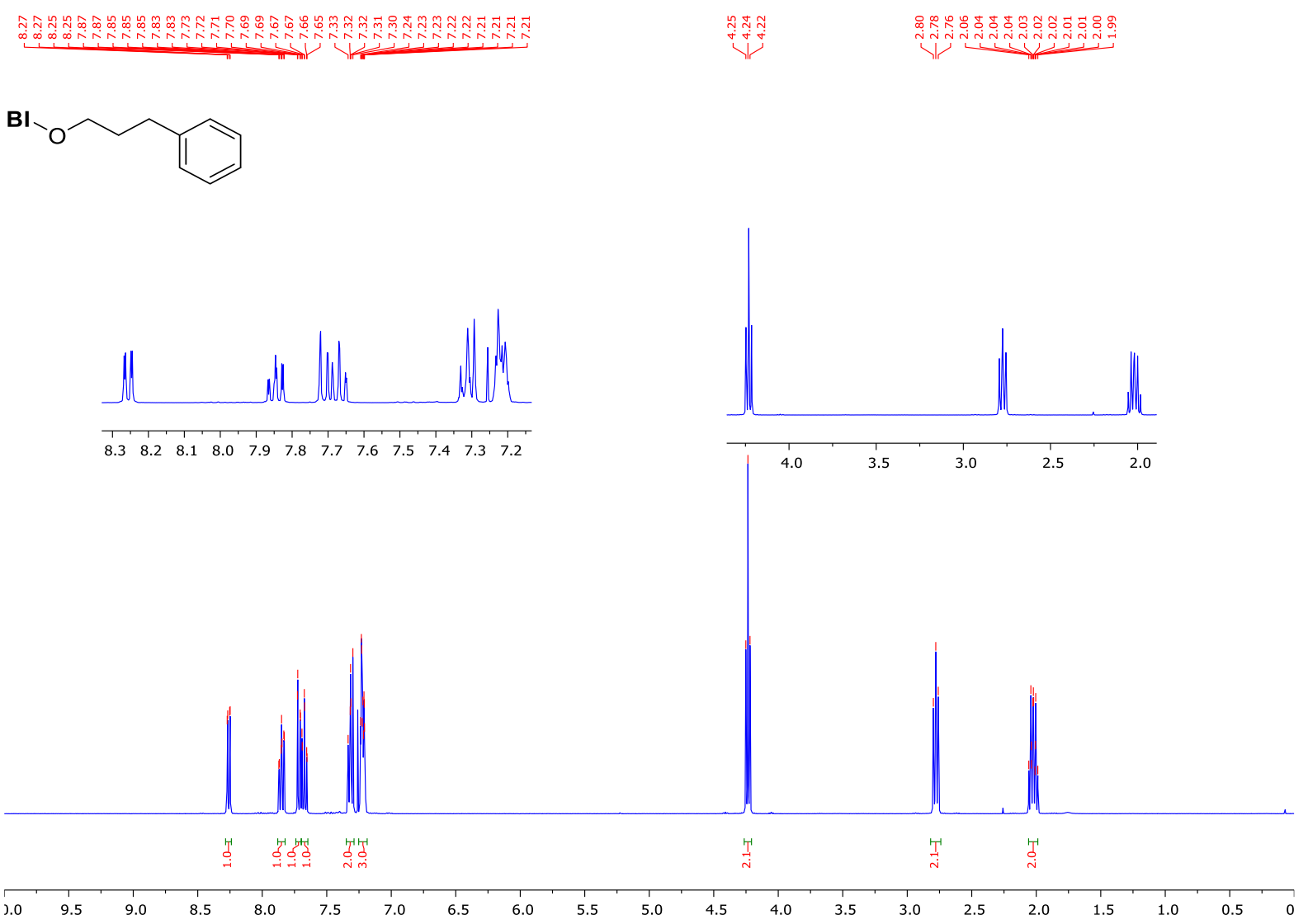

${ }^{13} \mathrm{C}$ NMR $\left(101 \mathrm{MHz}, \mathrm{CDCl}_{3}\right)$

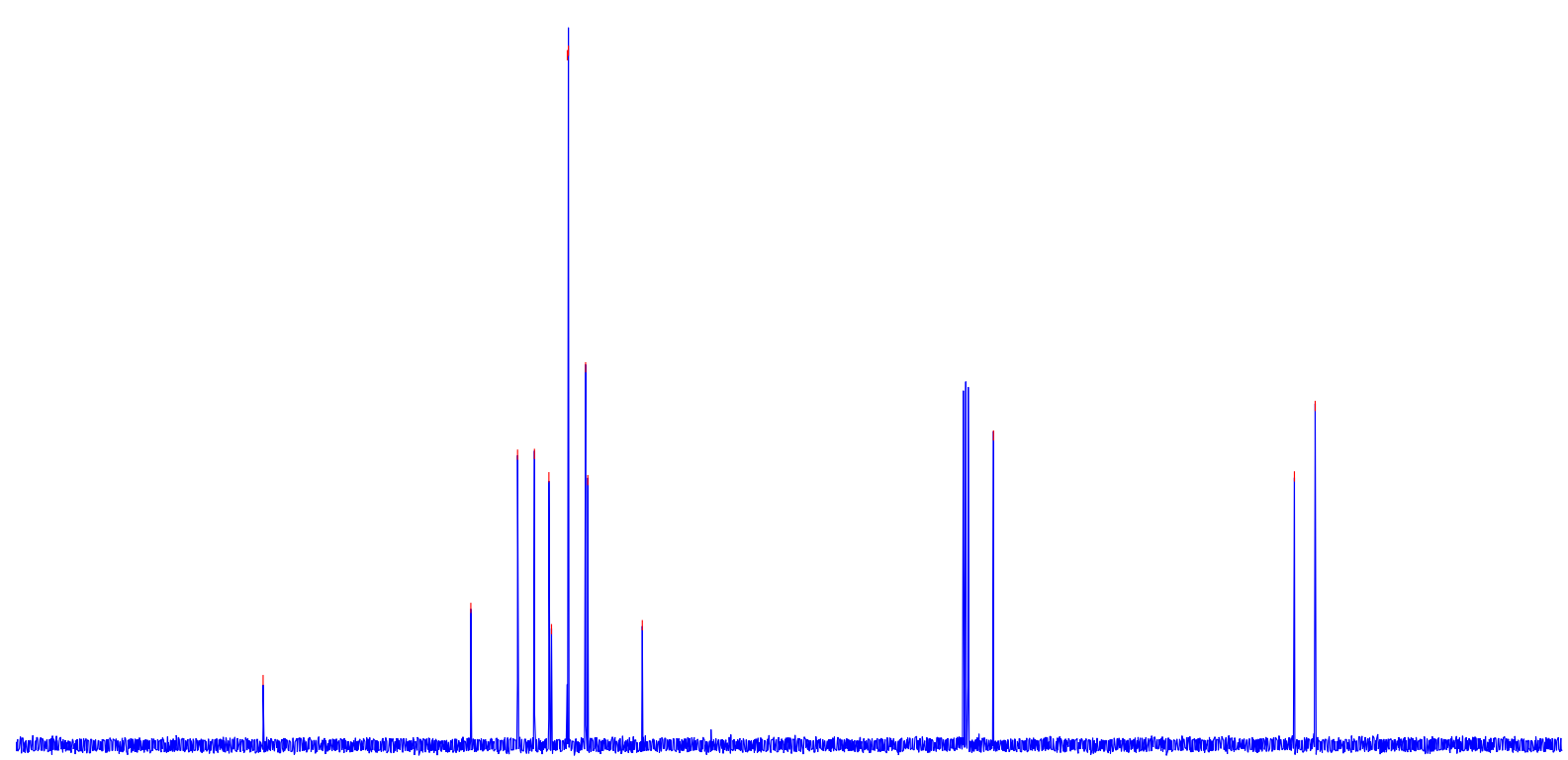

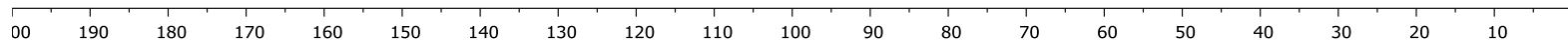


$\underline{\mathbf{2 m}},{ }^{1} \mathrm{H}$ NMR $\left(400 \mathrm{MHz}, \mathrm{CDCl}_{3}\right)$

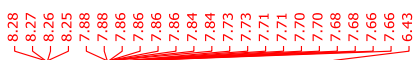<smiles>COc1cc(CCCOBr)cc(OC)c1OC</smiles>
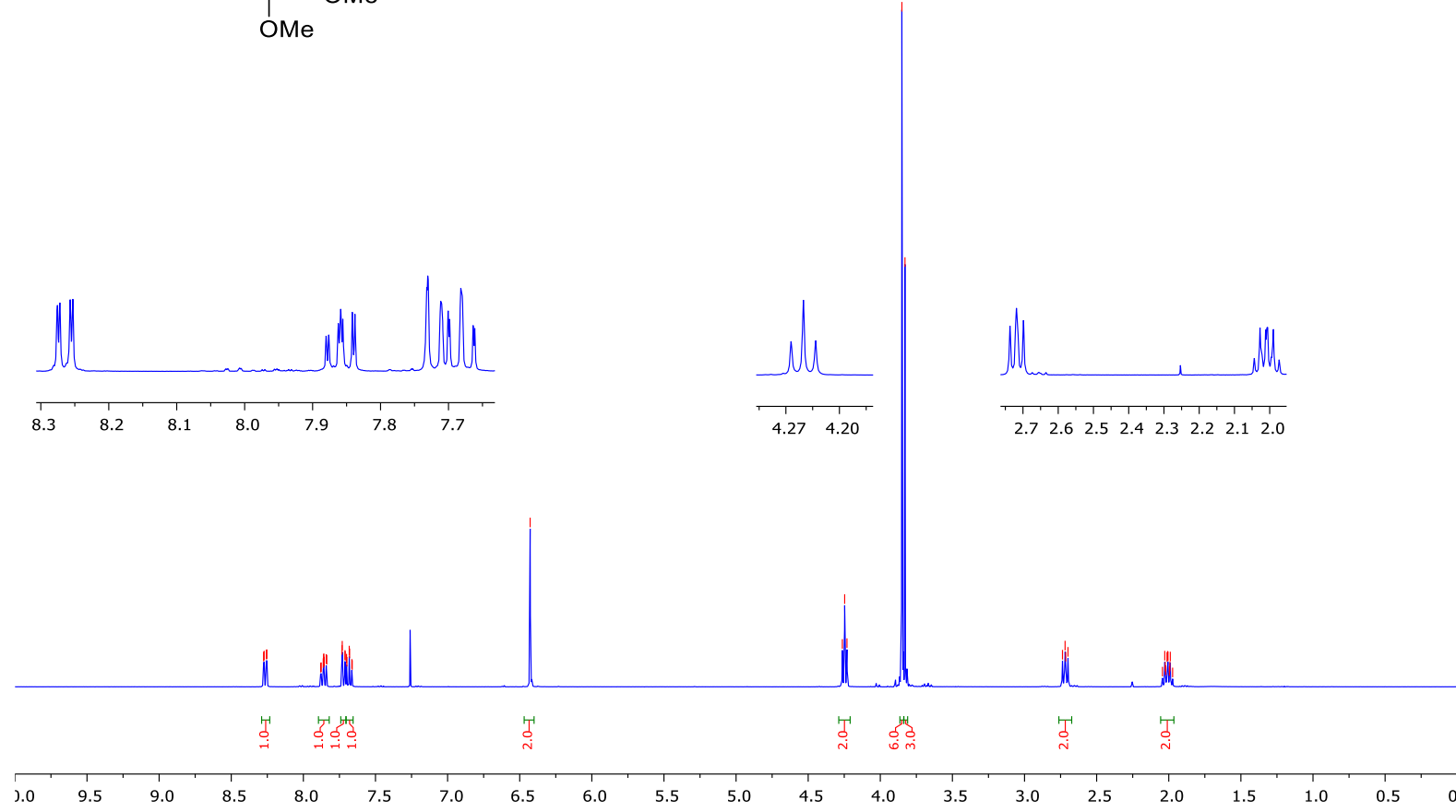

${ }^{13} \mathrm{C}$ NMR (101 MHz, $\left.\mathrm{CDCl}_{3}\right)$

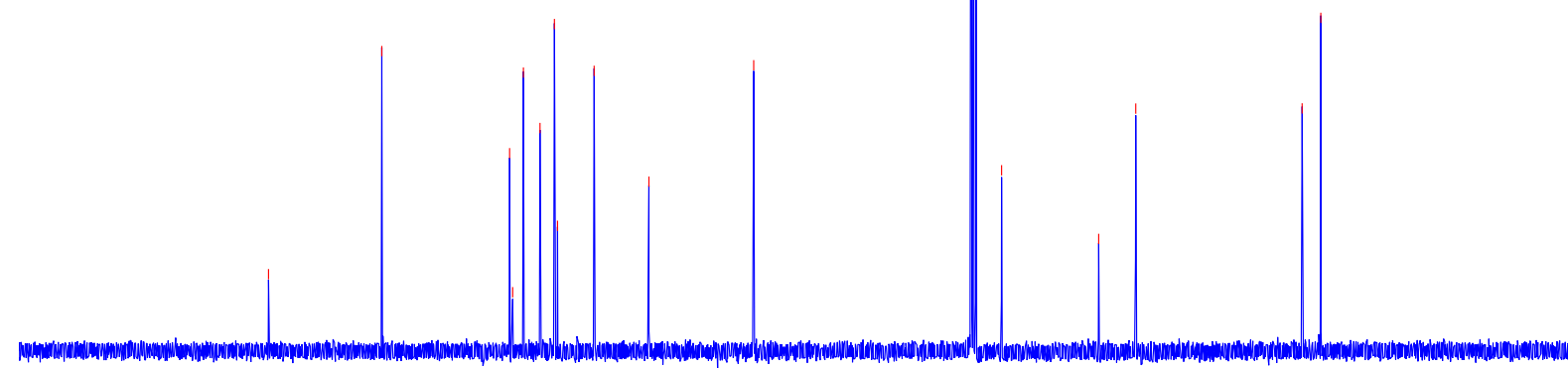


2n, ${ }^{1} \mathrm{H}$ NMR $\left(400 \mathrm{MHz}, \mathrm{CDCl}_{3}\right)$

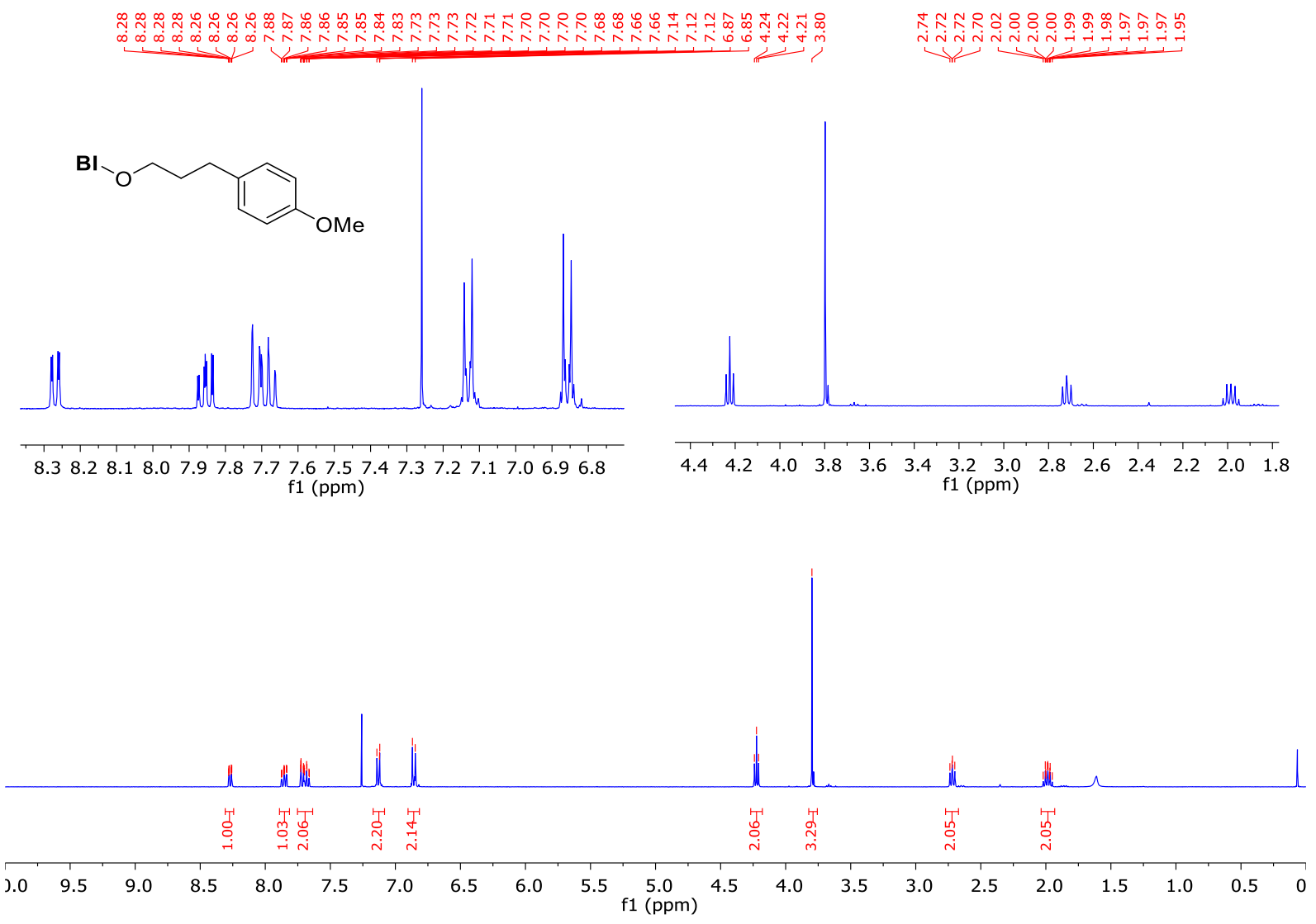

${ }^{13} \mathrm{C}$ NMR (101 MHz, $\left.\mathrm{CDCl}_{3}\right)$

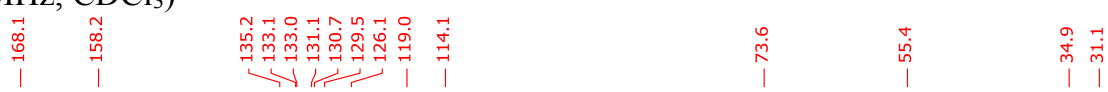

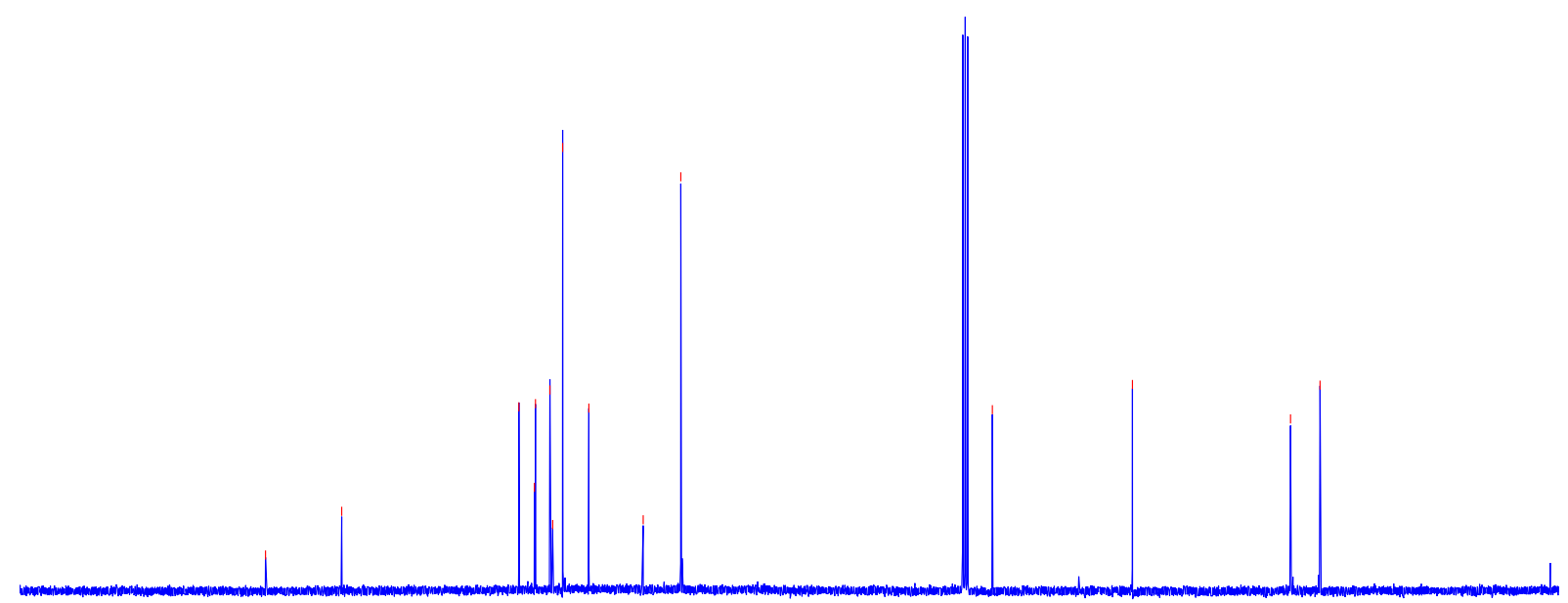

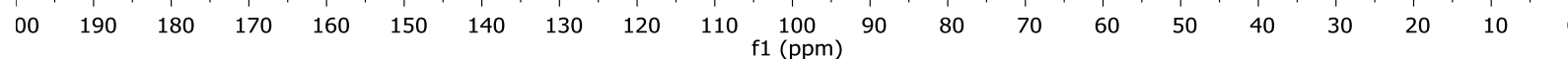


20, ${ }^{1} \mathrm{H}$ NMR $\left(400 \mathrm{MHz}, \mathrm{CDCl}_{3}\right)$

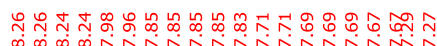

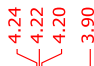

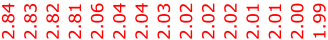

B<smiles>COC(=O)c1ccc(CCCOI)cc1</smiles>

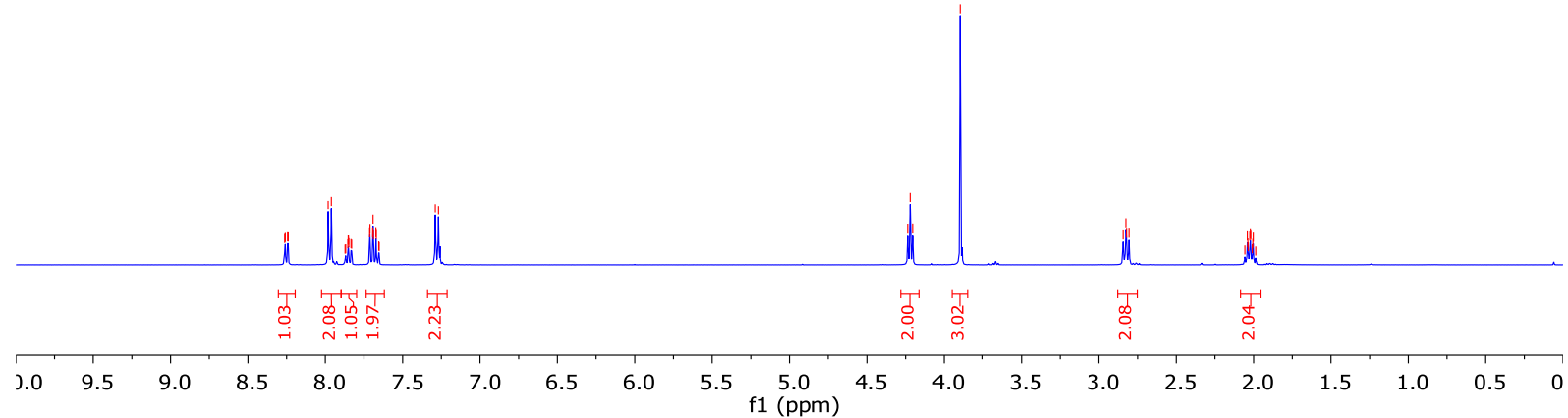

${ }^{13} \mathrm{C}$ NMR (101 MHz, $\left.\mathrm{CDCl}_{3}\right)$

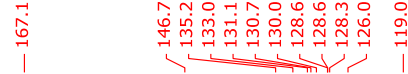

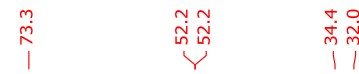

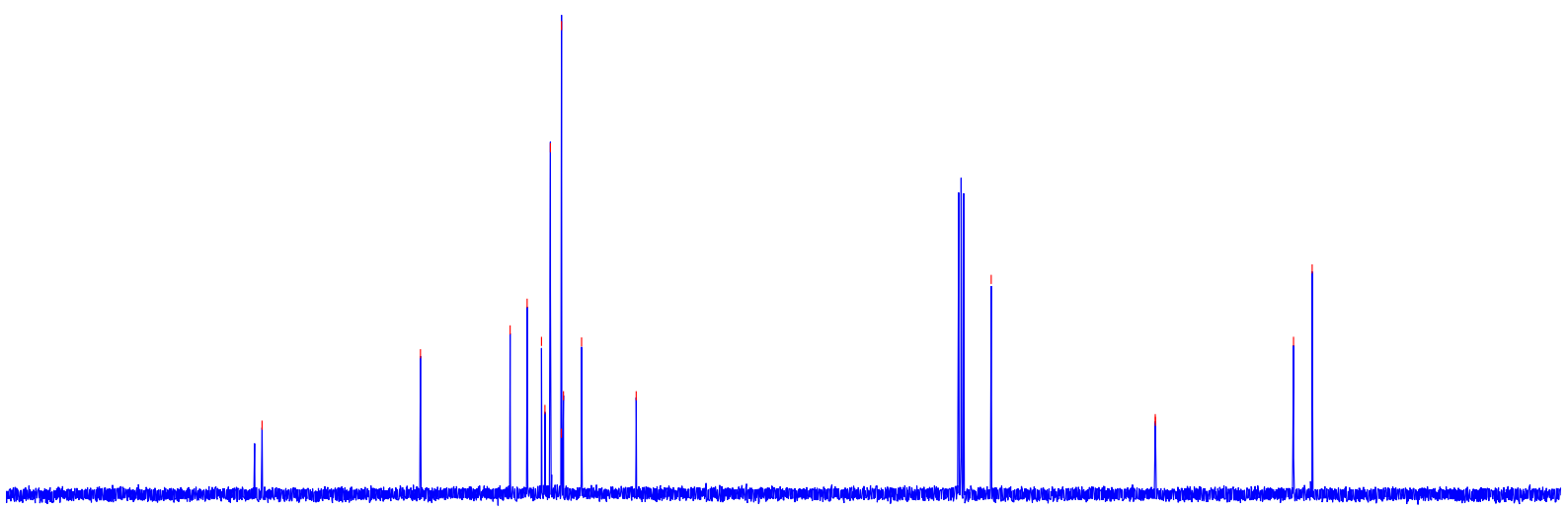

00

$\begin{array}{llll}90 & 180 & 170 & 160\end{array}$

$150 \quad 140$

130

$110 \underset{\mathrm{f} 1(\mathrm{ppm})}{100} 90 \quad 80$

$\begin{array}{ll}70 & 60\end{array}$

$50 \quad 40$

$\begin{array}{lll}30 & 20 \quad 10\end{array}$ 
2pp, ${ }^{1} \mathrm{H}$ NMR $\left(400 \mathrm{MHz}, \mathrm{CDCl}_{3}\right)$

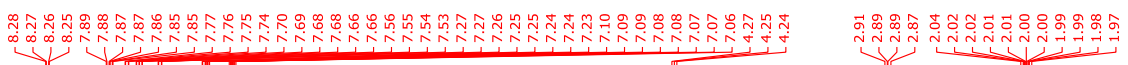<smiles>BrOCCCc1ccccc1Br</smiles>
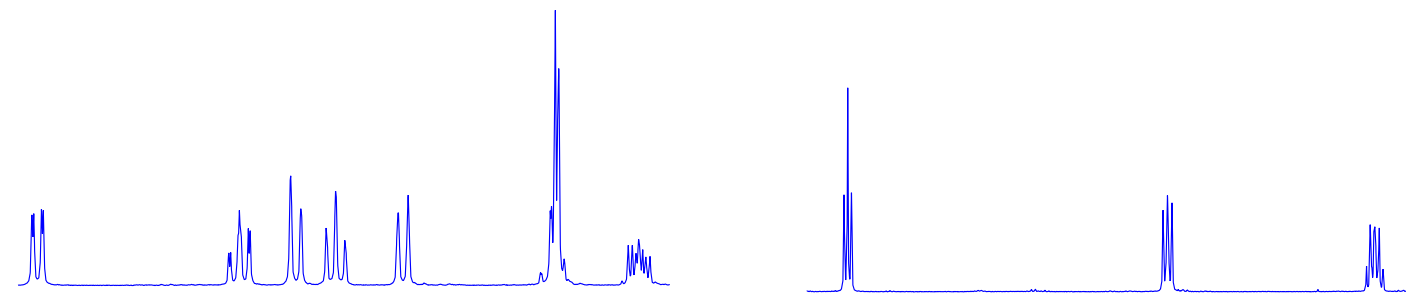

\begin{tabular}{lllllllllllllll}
\hline & 8.3 & 8.2 & 8.1 & 8.0 & 7.9 & 7.8 & 7.7 & 7.6 & 7.5 & 7.4 & 7.3 & 7.2 & 7.1
\end{tabular}
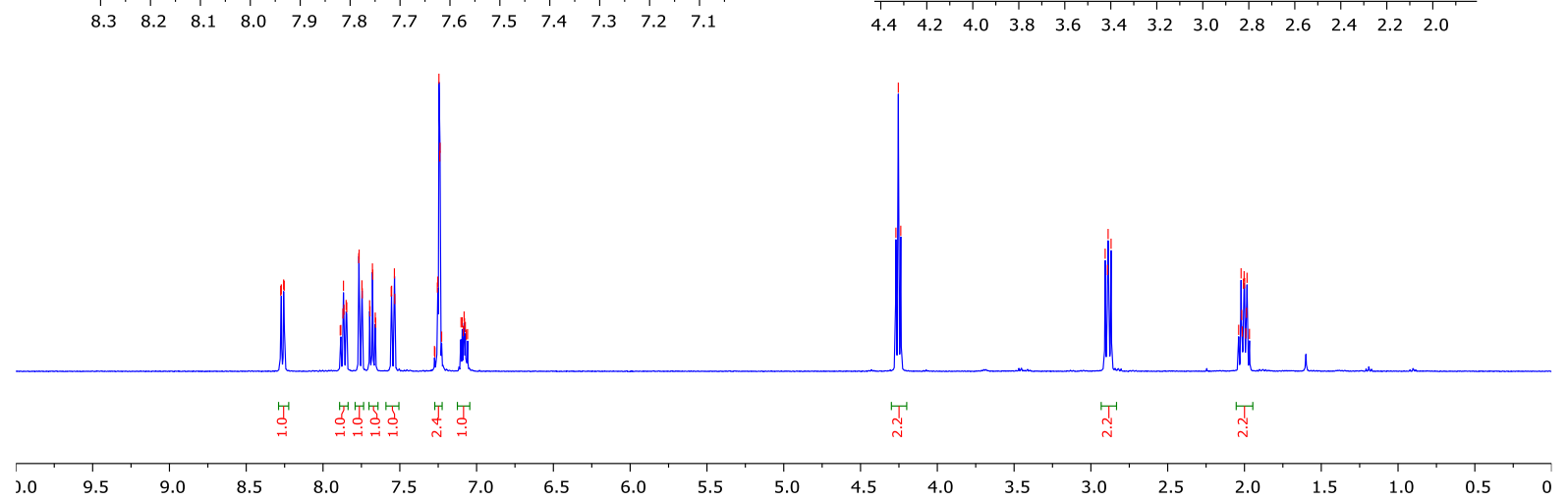

${ }^{13} \mathrm{C}$ NMR (101 MHz, $\left.\mathrm{CDCl}_{3}\right)$

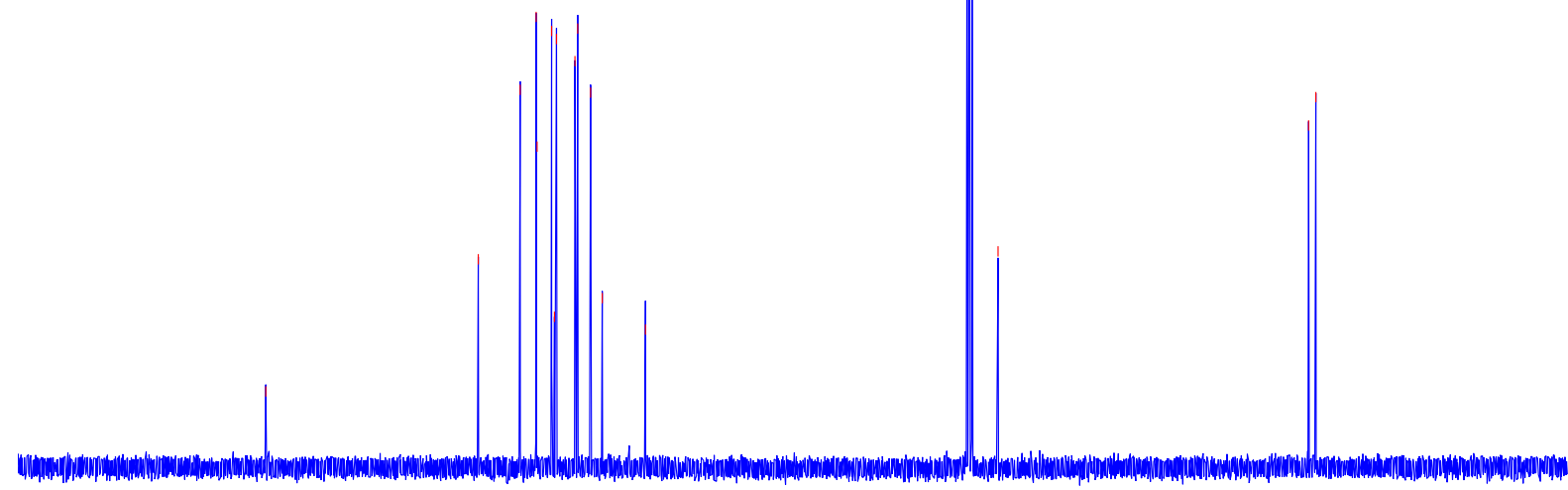

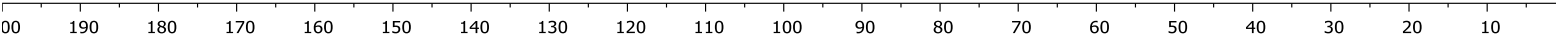


2q, ${ }^{1} \mathrm{H}$ NMR $\left(400 \mathrm{MHz}, \mathrm{CDCl}_{3}\right)$

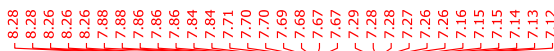

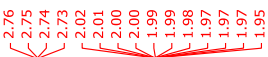

BI<smiles>Clc1ccc(CCCOI)cc1</smiles>
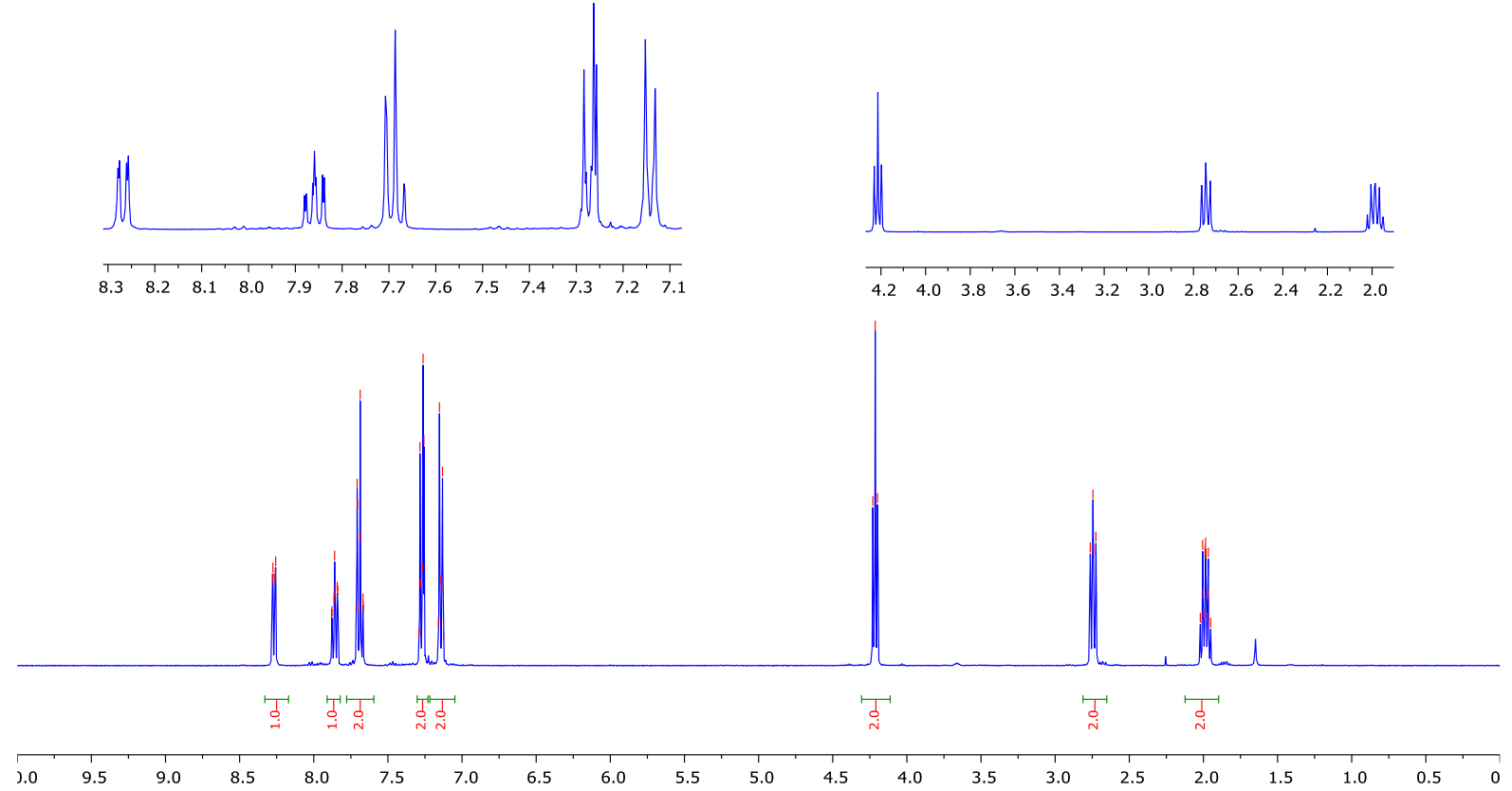

${ }^{13} \mathrm{C}$ NMR (101 MHz, $\left.\mathrm{CDCl}_{3}\right)$

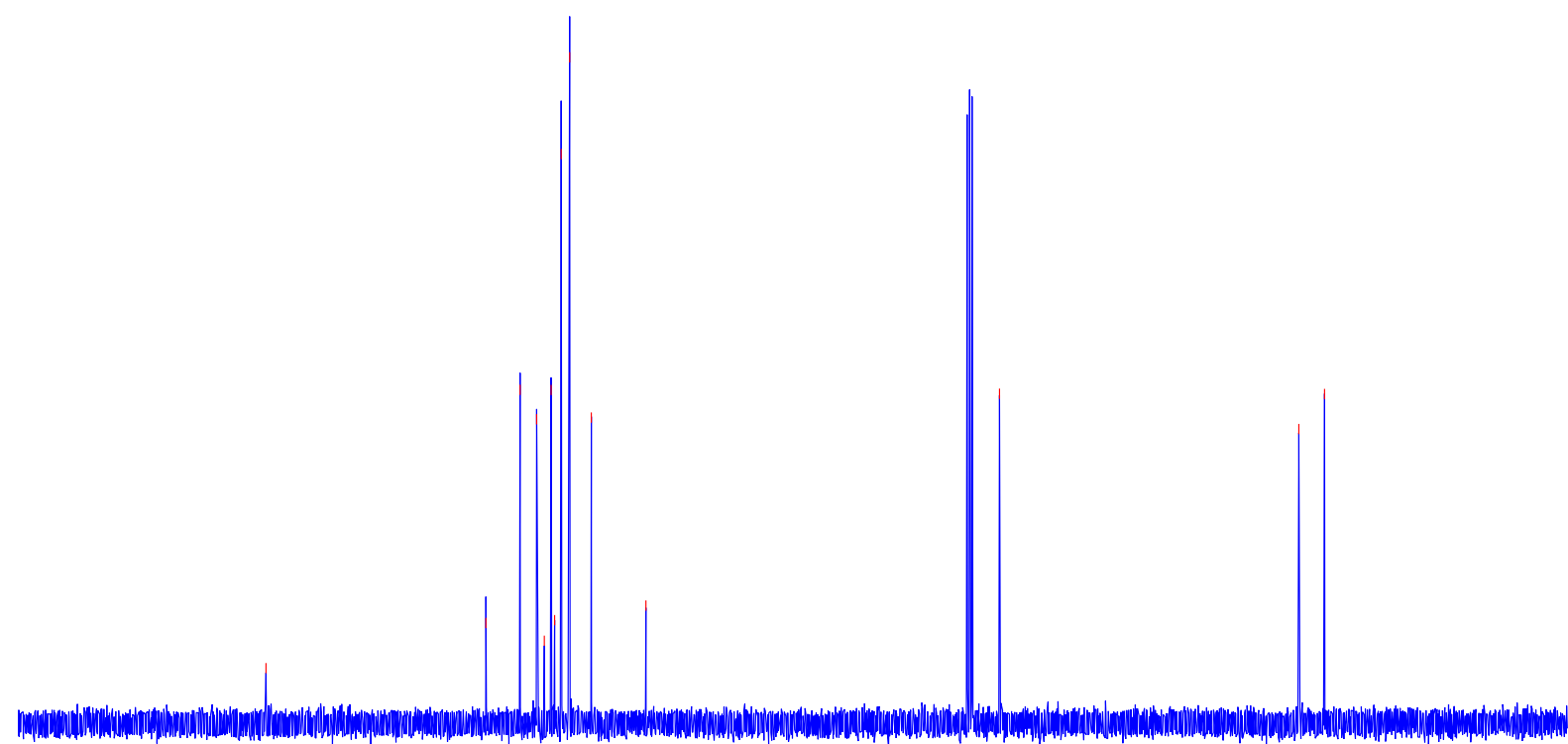

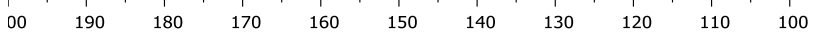
$90 \quad 80$

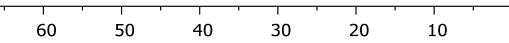


$\underline{\mathbf{2 r}},{ }^{1} \mathrm{H} \mathrm{NMR}\left(400 \mathrm{MHz}, \mathrm{CDCl}_{3}\right)$

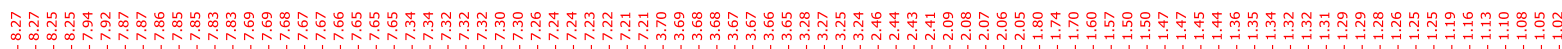

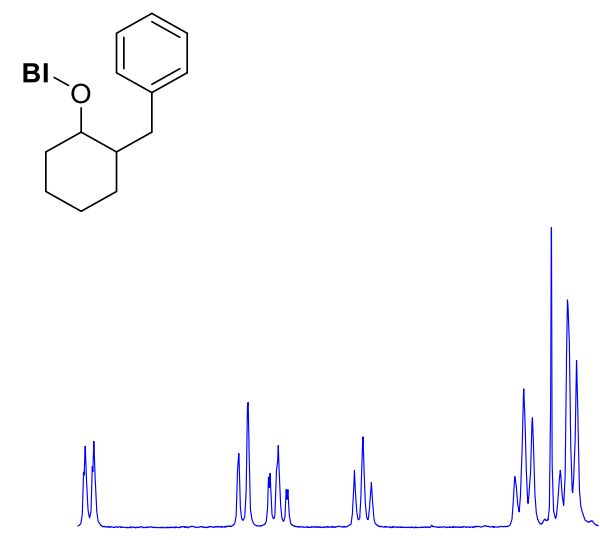

$\begin{array}{llllllllllllll}8.2 & 8.1 & 8.0 & 7.9 & 7.8 & 7.7 & 7.6 & 7.5 & 7.4 & 7.3 & 7.2\end{array}$
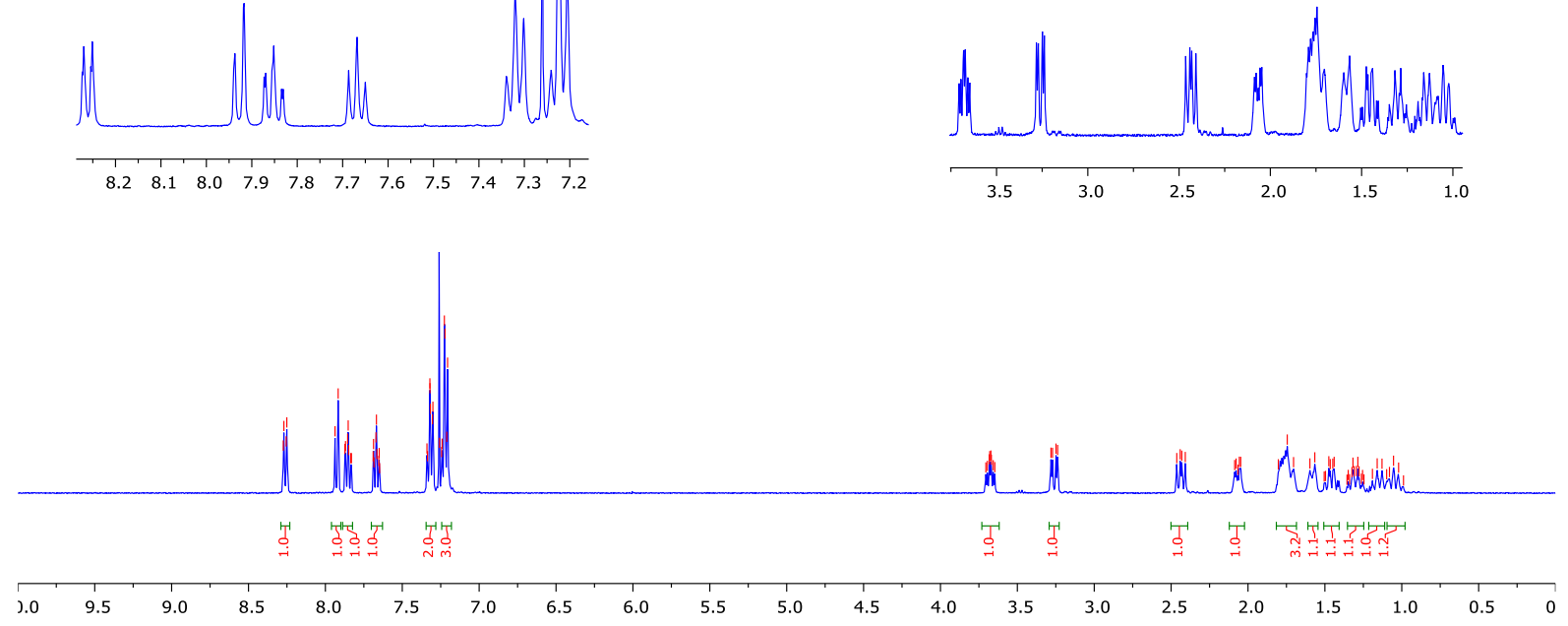

${ }^{13} \mathrm{C}$ NMR $\left(101 \mathrm{MHz}, \mathrm{CDCl}_{3}\right)$

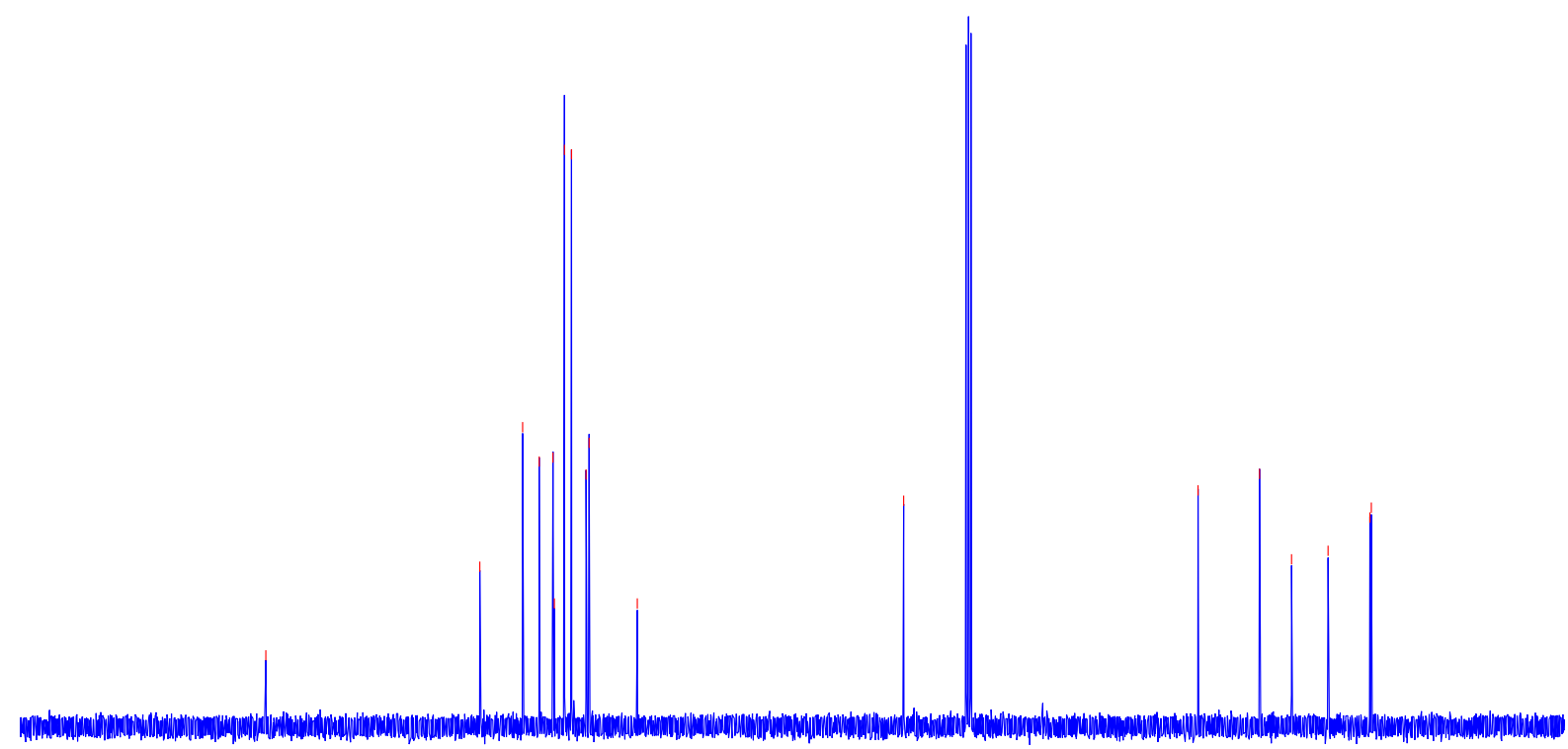

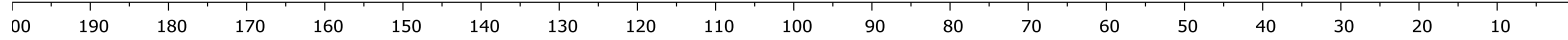


$\underline{\text { 2s }},{ }^{1} \mathrm{H}$ NMR (400 MHz, $\left.\mathrm{CDCl}_{3}\right)$

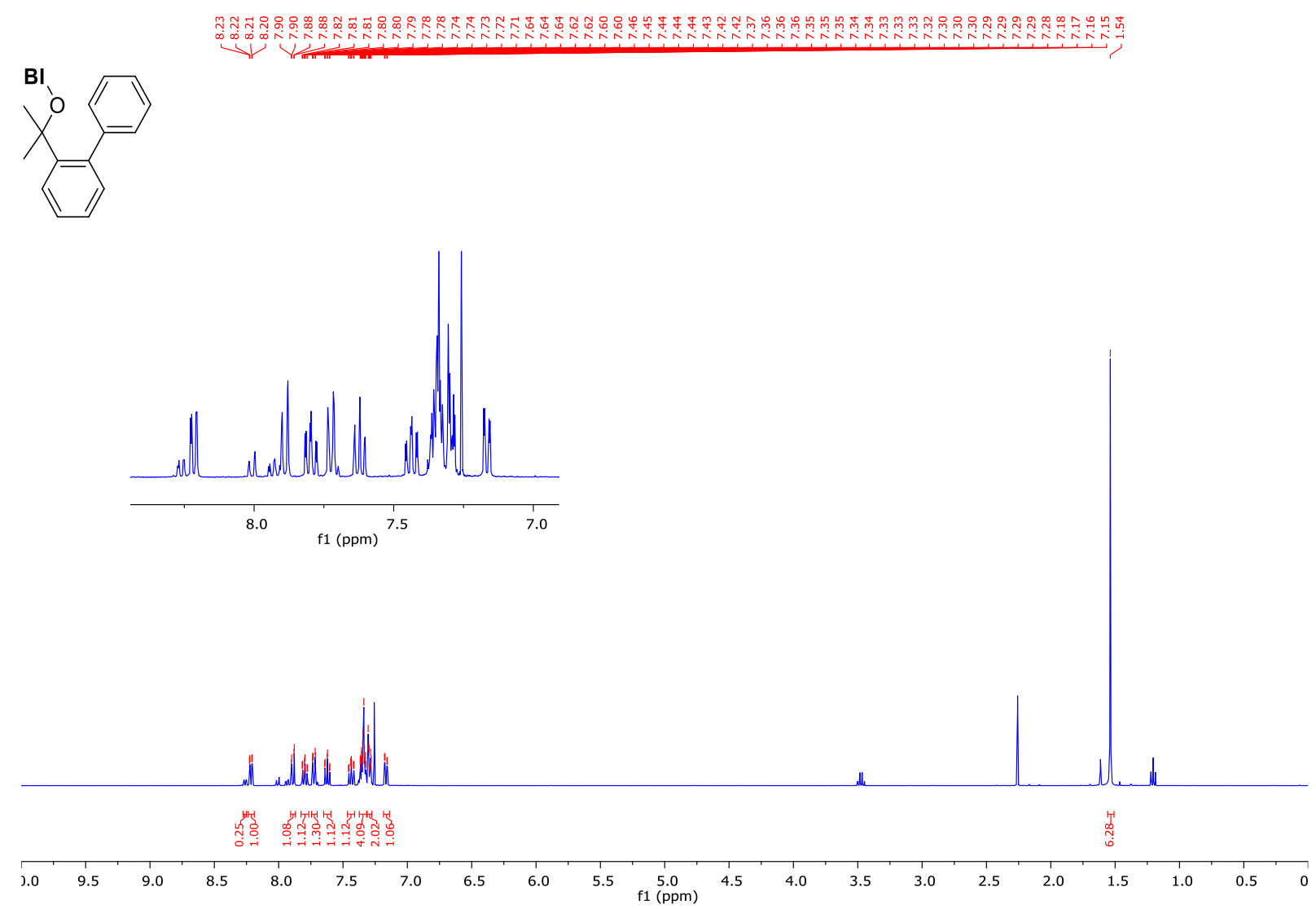

${ }^{13} \mathrm{C}$ NMR $\left(101 \mathrm{MHz}, \mathrm{CDCl}_{3}\right)$

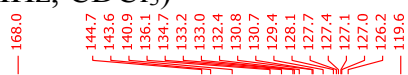

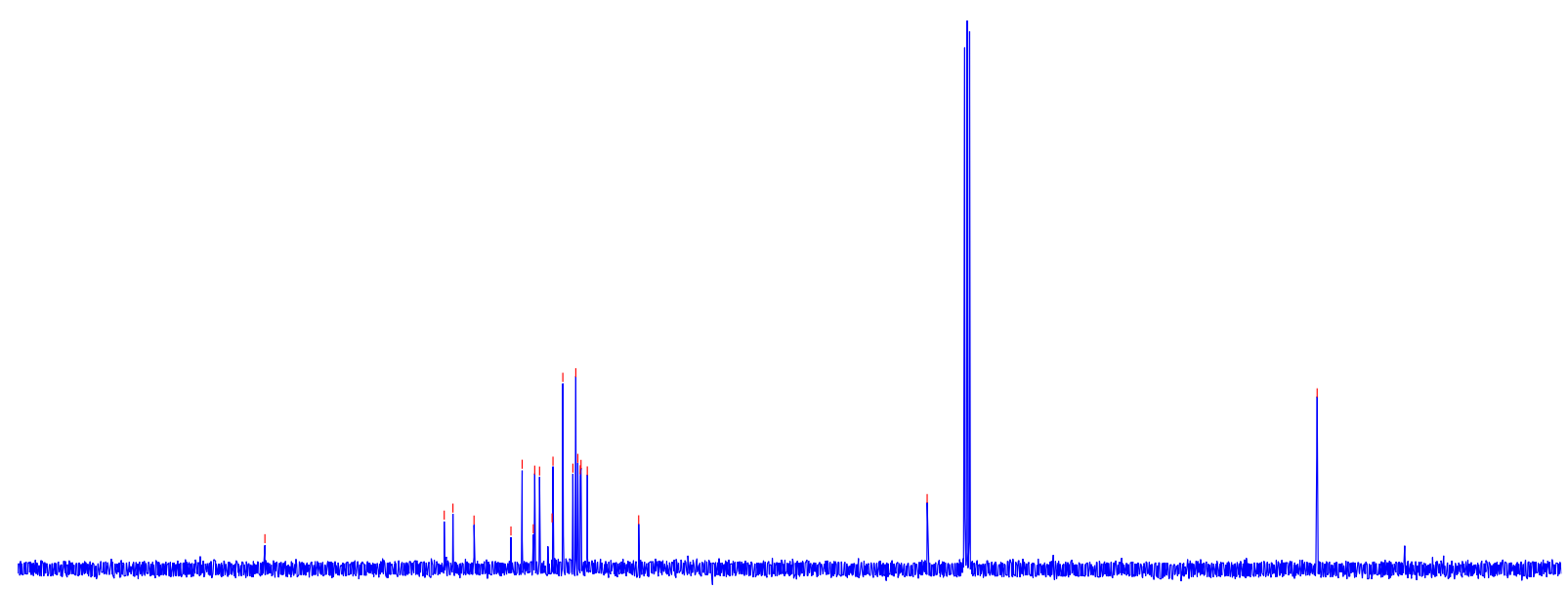

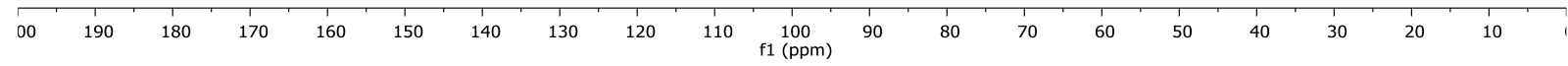


$\underline{\text { 2tt }},{ }^{1} \mathrm{H}$ NMR $\left(400 \mathrm{MHz}, \mathrm{CDCl}_{3}\right)$

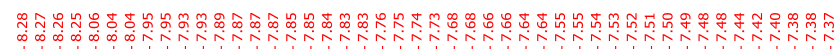

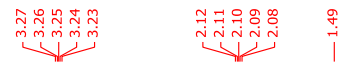

B1
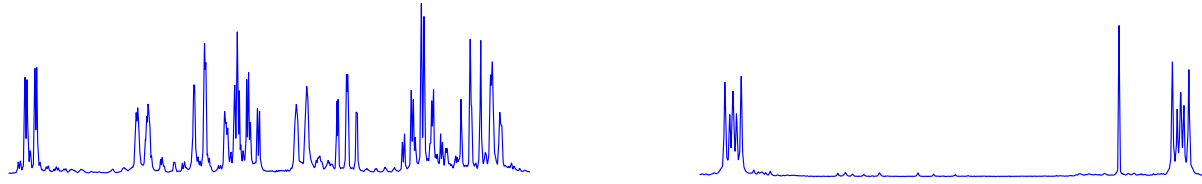

$\begin{array}{lllllllllll}8.3 & 8.2 & 8.1 & 8.0 & 7.9 & 7.8 & 7.7 & 7.6 & 7.5 & 7.4\end{array}$

$\begin{array}{lllllllllllllll}3.3 & 3.2 & 3.1 & 3.0 & 2.9 & 2.8 & 2.7 & 2.6 & 2.5 & 2.4 & 2.3 & 2.2 & 2.1 & 2\end{array} .0$

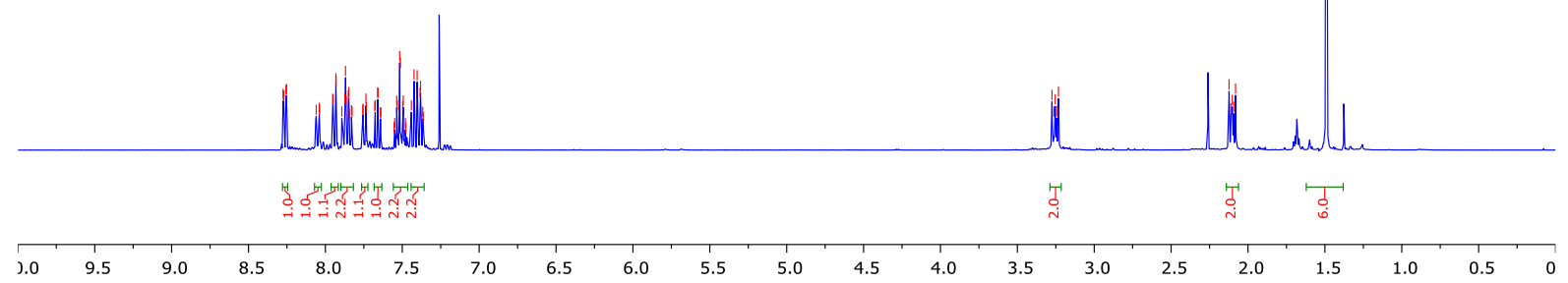

${ }^{13} \mathrm{C}$ NMR (101 MHz, $\left.\mathrm{CDCl}_{3}\right)$

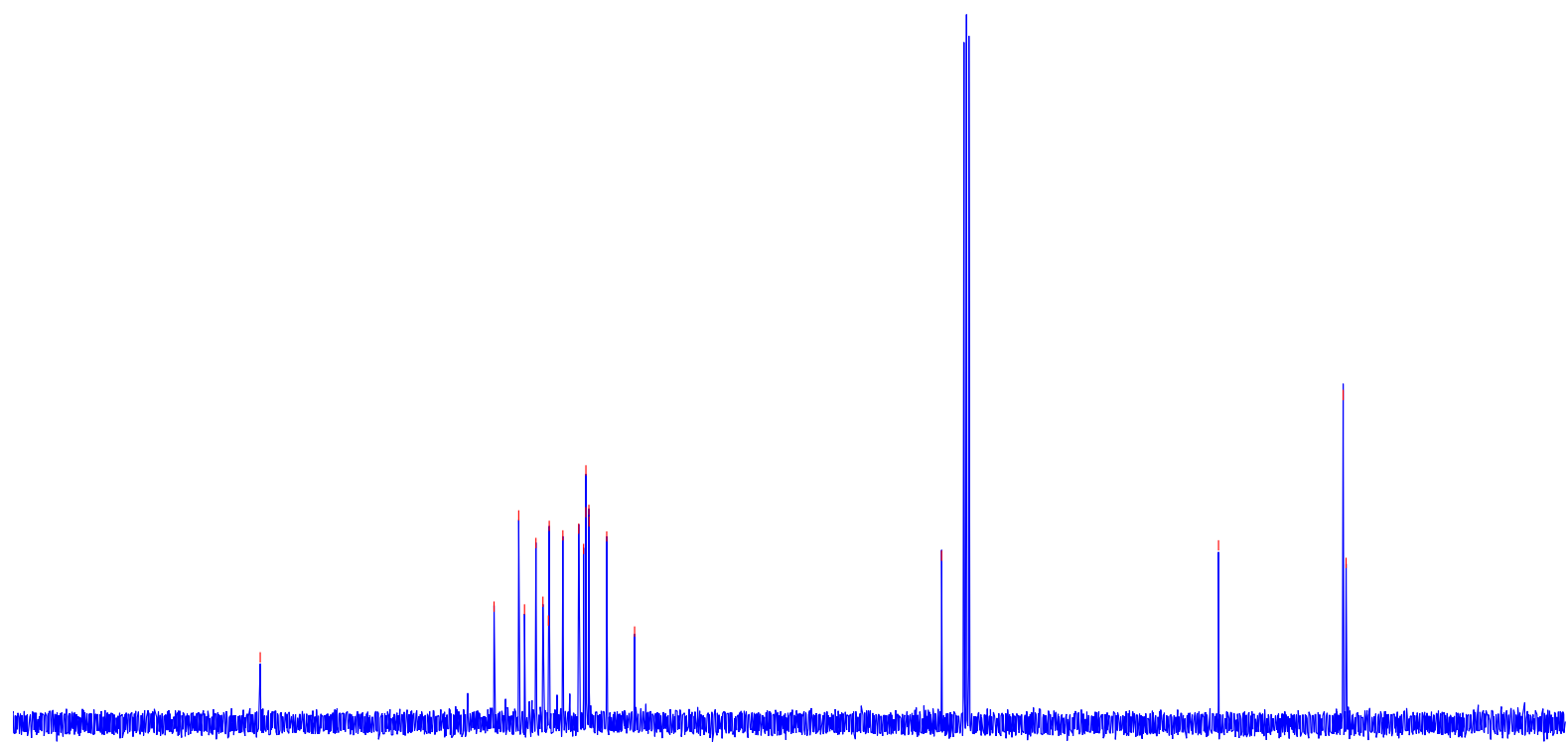

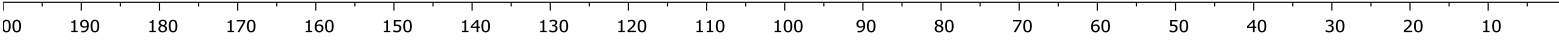


$\underline{\mathbf{2 u}},{ }^{1} \mathrm{H}$ NMR $\left(400 \mathrm{MHz}, \mathrm{CDCl}_{3}\right)$

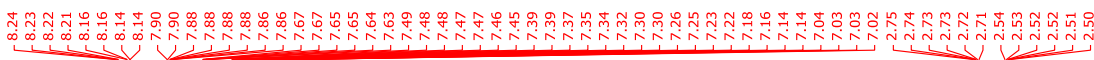

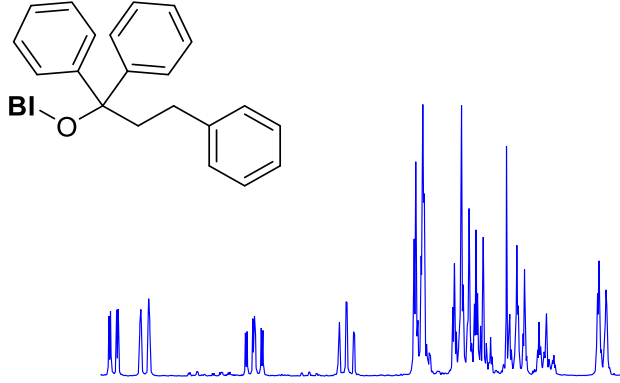

$\begin{array}{lllllllllllllllllllll}8.2 & 8.1 & 8.0 & 7.9 & 7.8 & 7.7 & 7.6 & 7.5 & 7.4 & 7.3 & 7.2 & 7.1 & 7.0\end{array}$

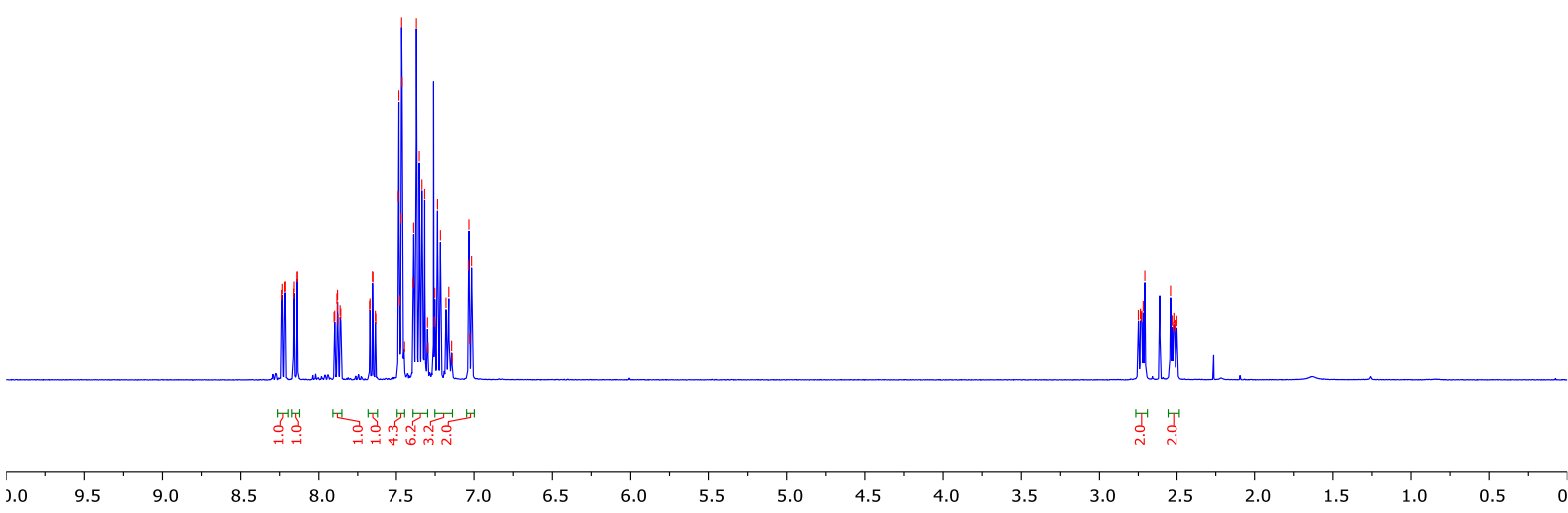

${ }^{13} \mathrm{C}$ NMR (101 MHz, $\left.\mathrm{CDCl}_{3}\right)$

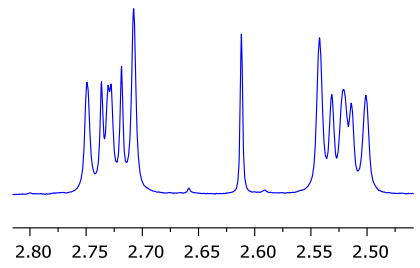

$\begin{array}{lllllll}2.80 & 2.75 & 2.70 & 2.65 & 2.60 & 2.55 & 2.50\end{array}$

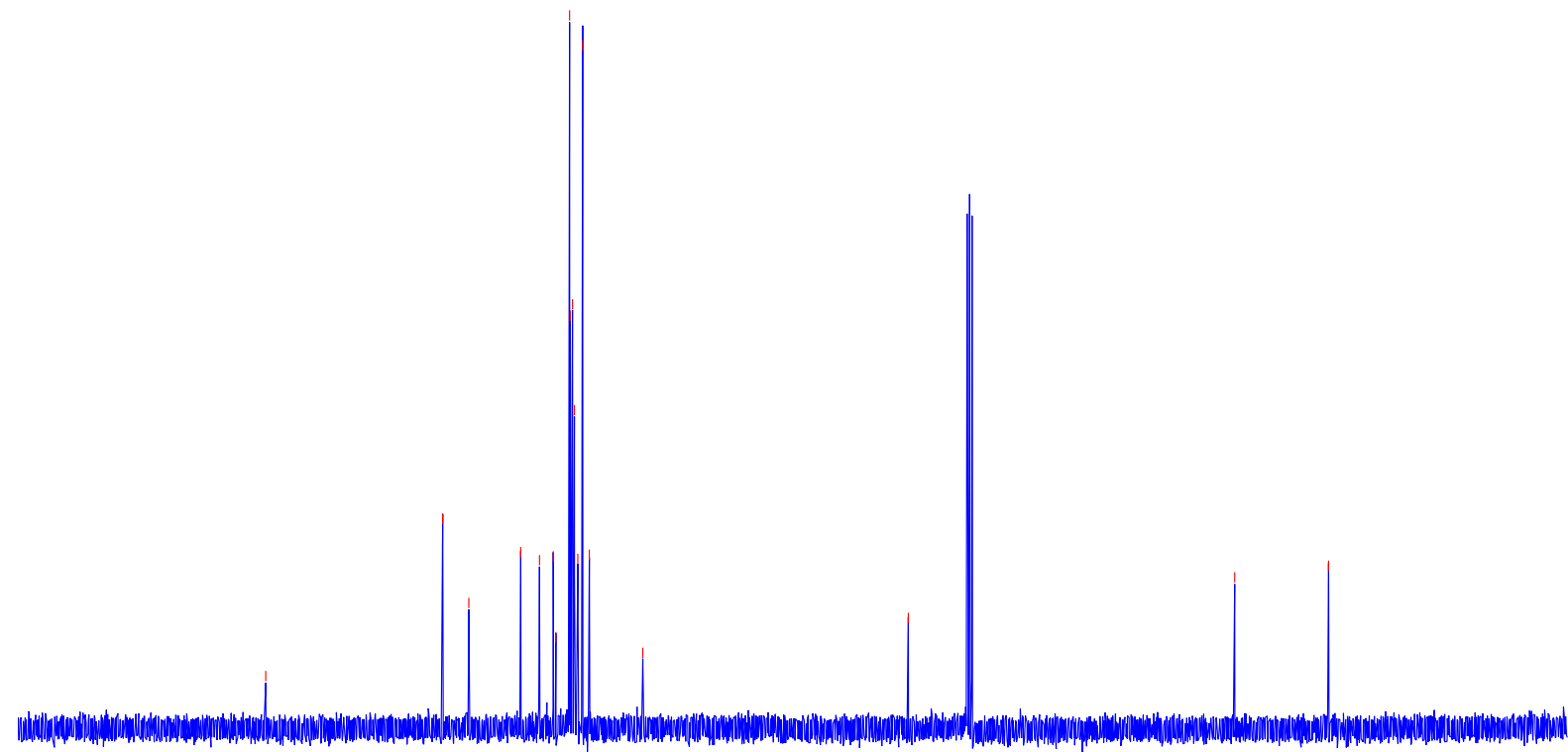

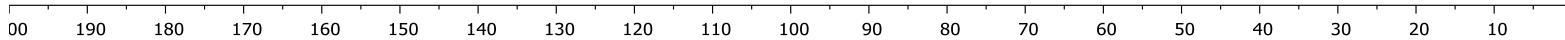


3a, ${ }^{1} \mathrm{H}$ NMR $\left(400 \mathrm{MHz}, \mathrm{CDCl}_{3}\right)$

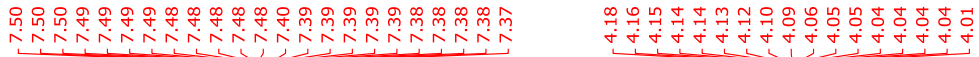

1
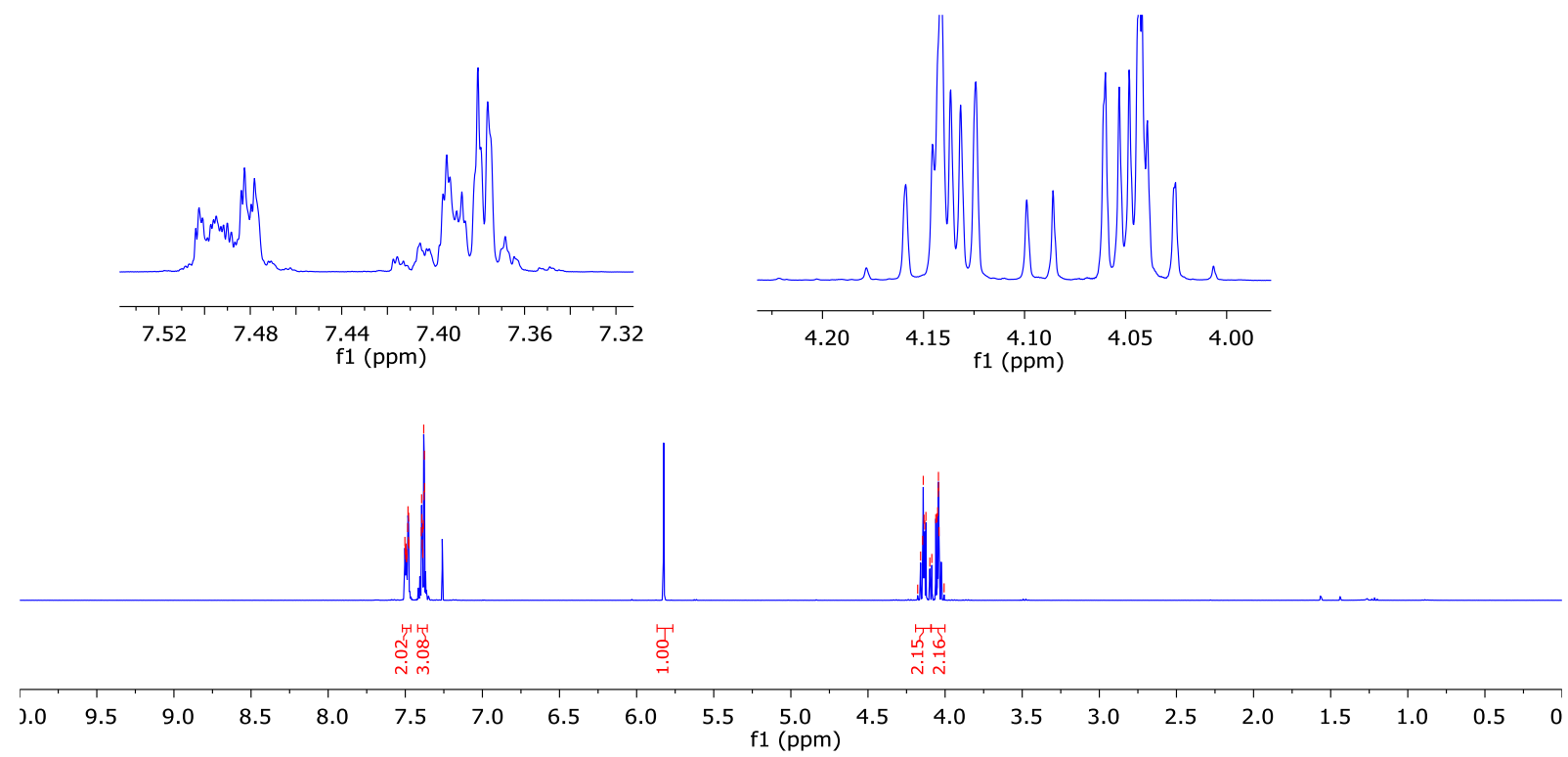

${ }^{13} \mathrm{C}$ NMR (101 MHz, $\left.\mathrm{CDCl}_{3}\right)$

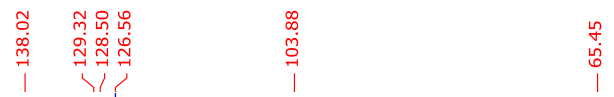

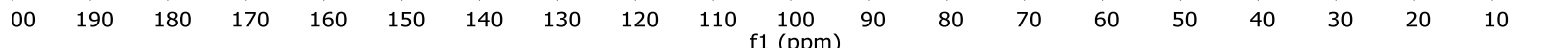


3c, ${ }^{1} \mathrm{H}$ NMR $\left(400 \mathrm{MHz}, \mathrm{CDCl}_{3}\right)$

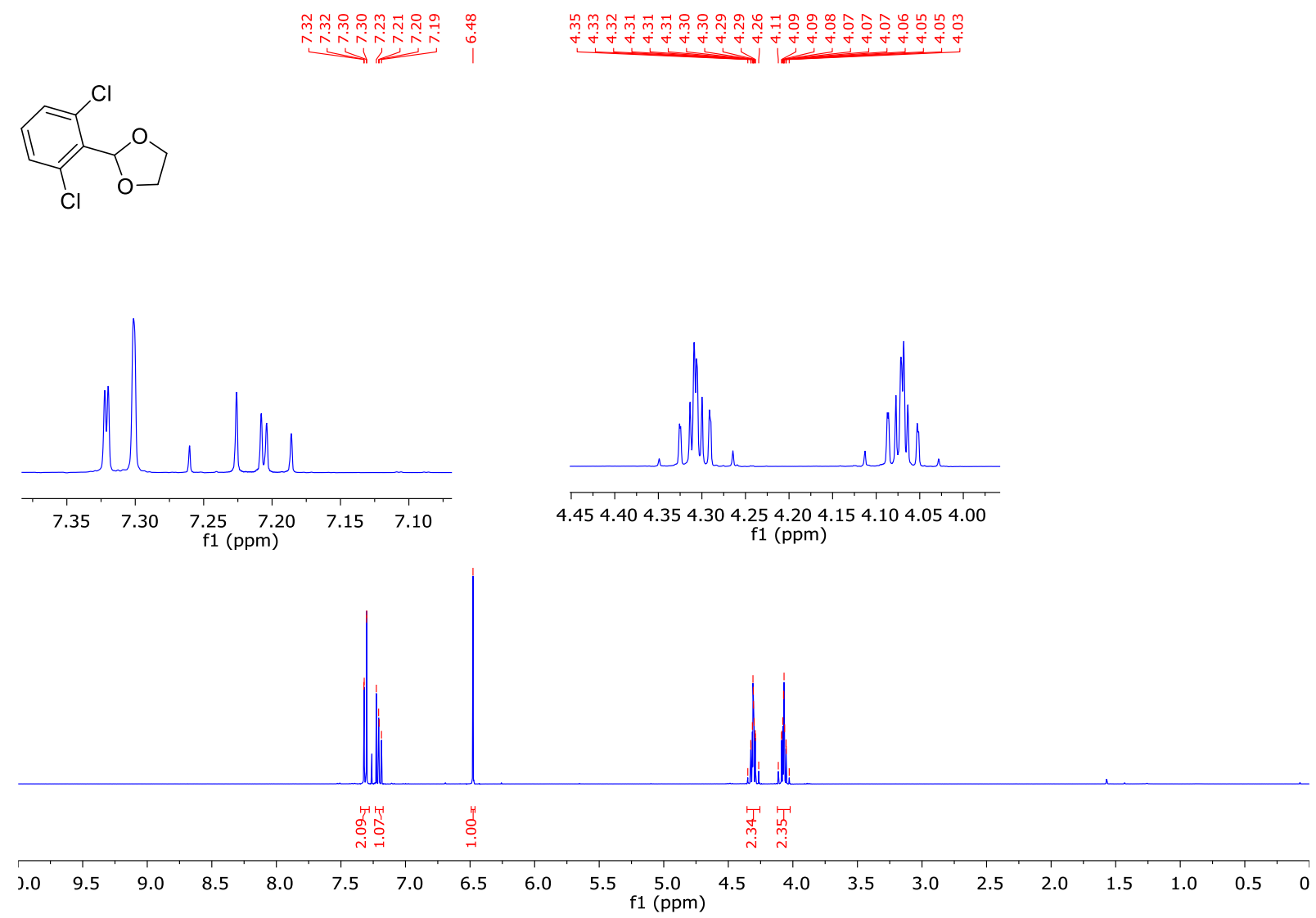

${ }^{13} \mathrm{C}$ NMR $\left(101 \mathrm{MHz}, \mathrm{CDCl}_{3}\right)$

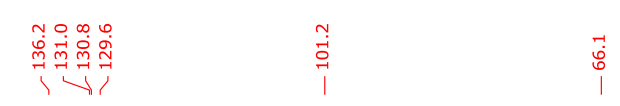

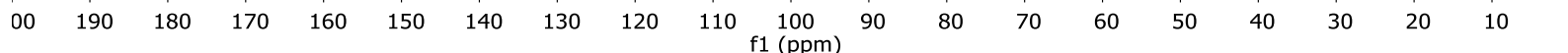


色, ${ }^{1} \mathrm{H}$ NMR $\left(400 \mathrm{MHz}, \mathrm{CDCl}_{3}\right)$<smiles>C1CCC2(CC1)OCCO2</smiles>

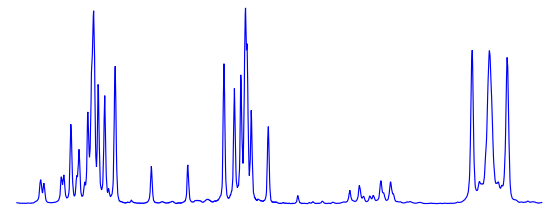

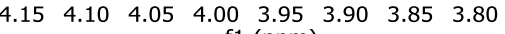
f1 (ppm)
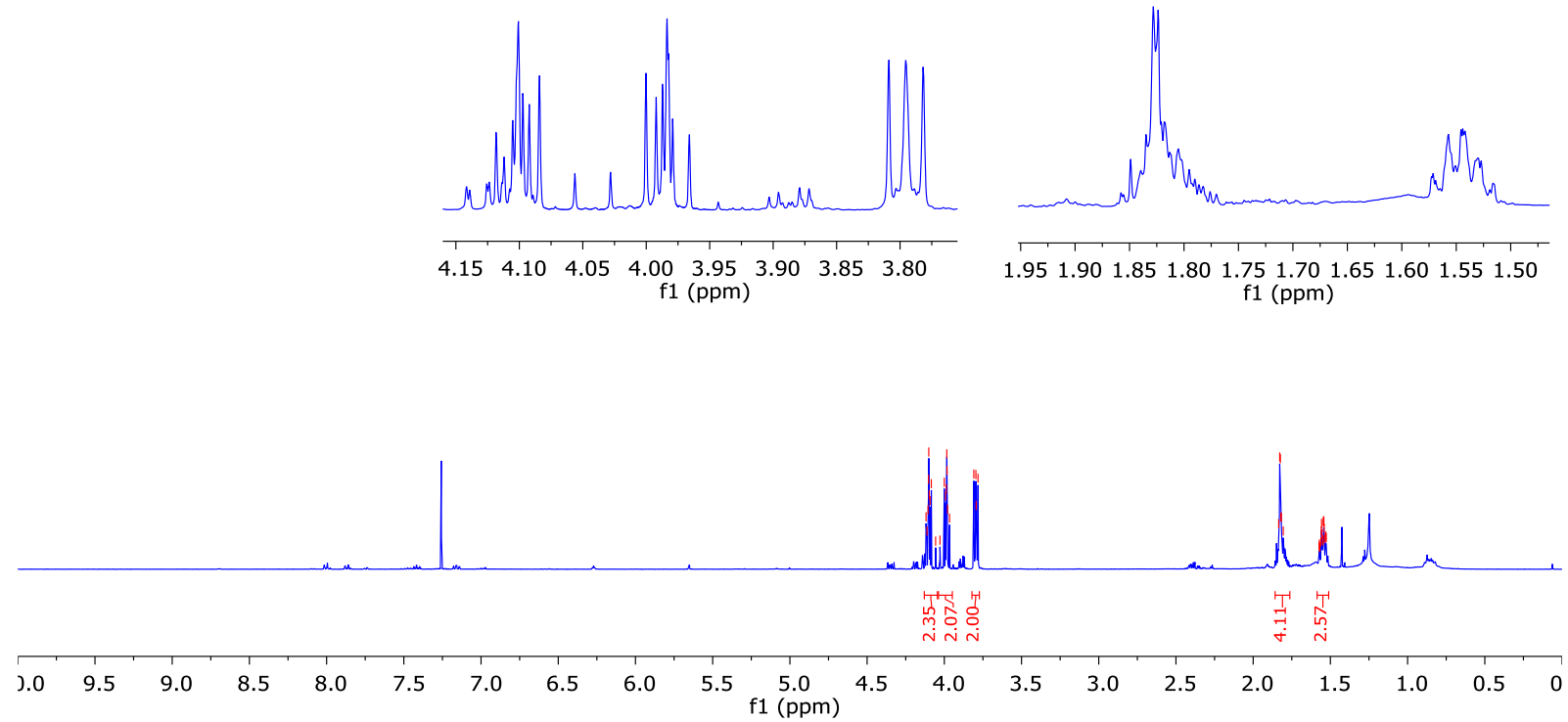

${ }^{13} \mathrm{C}$ NMR $\left(101 \mathrm{MHz}, \mathrm{CDCl}_{3}\right)$

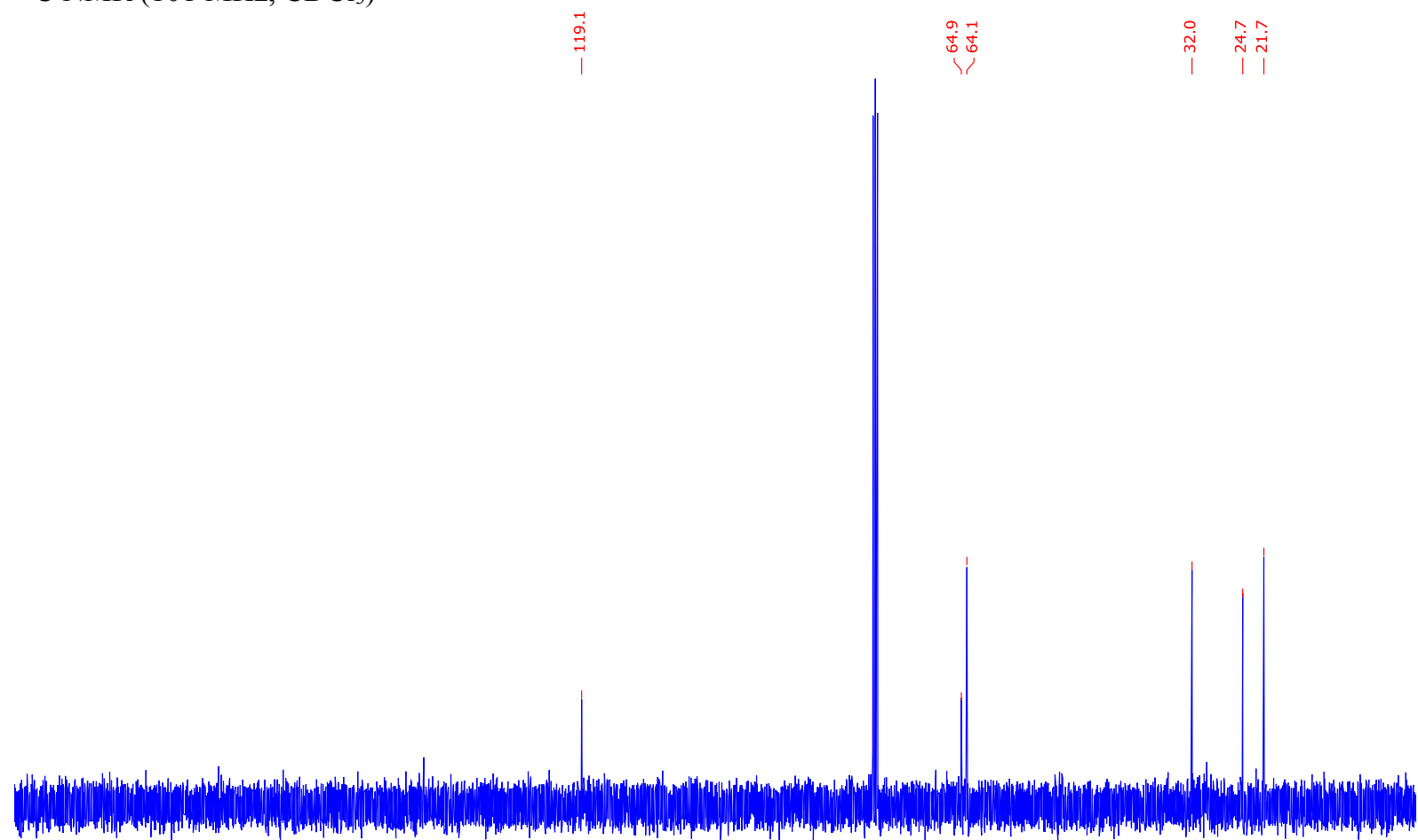

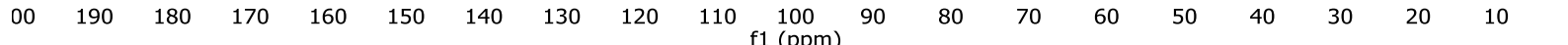




\section{3f, ${ }^{1} \mathrm{H}$ NMR $\left(400 \mathrm{MHz}, \mathrm{CDCl}_{3}\right)$}

9
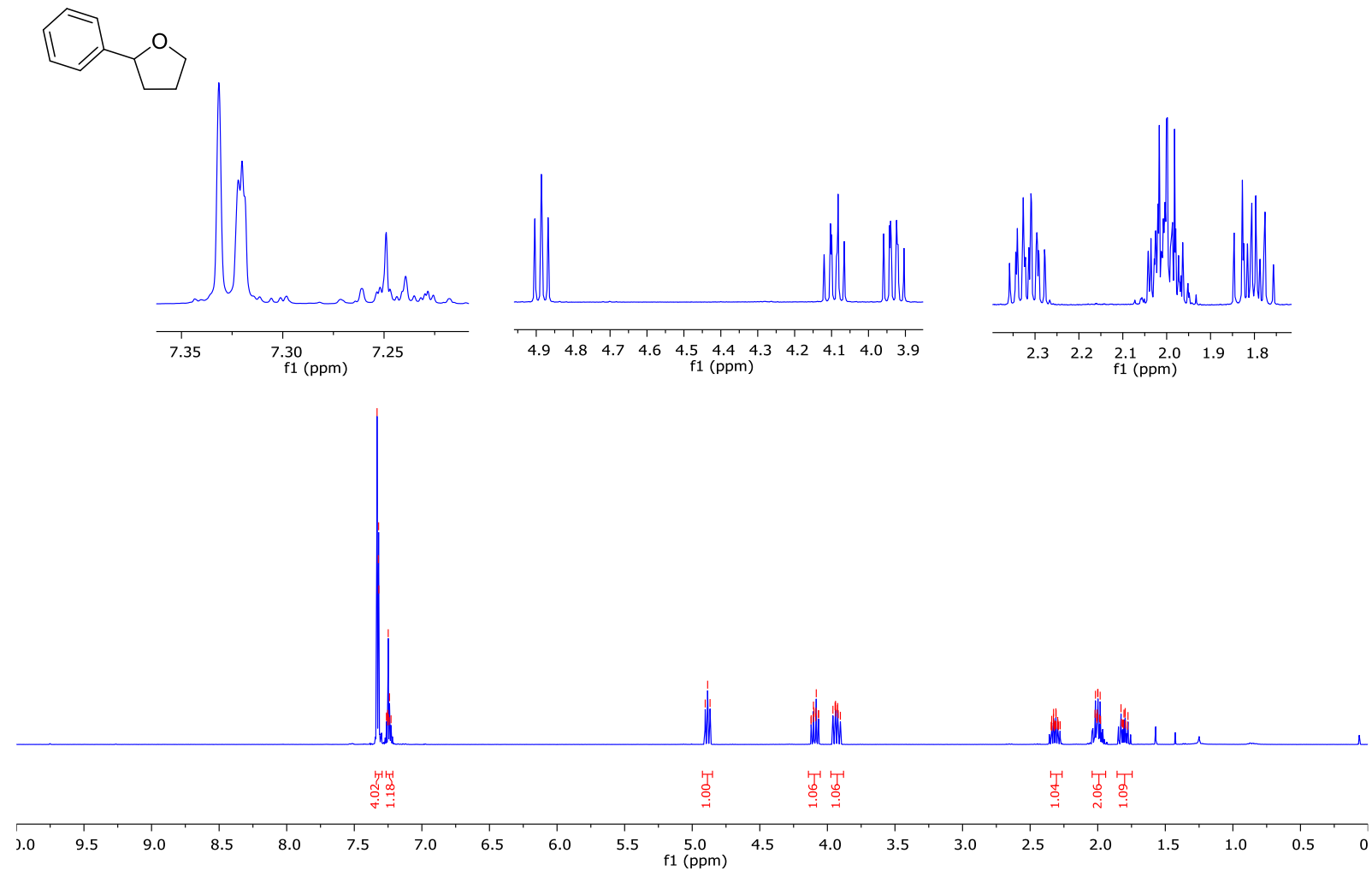

${ }^{13} \mathrm{C}$ NMR $\left(101 \mathrm{MHz}, \mathrm{CDCl}_{3}\right)$

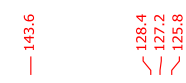

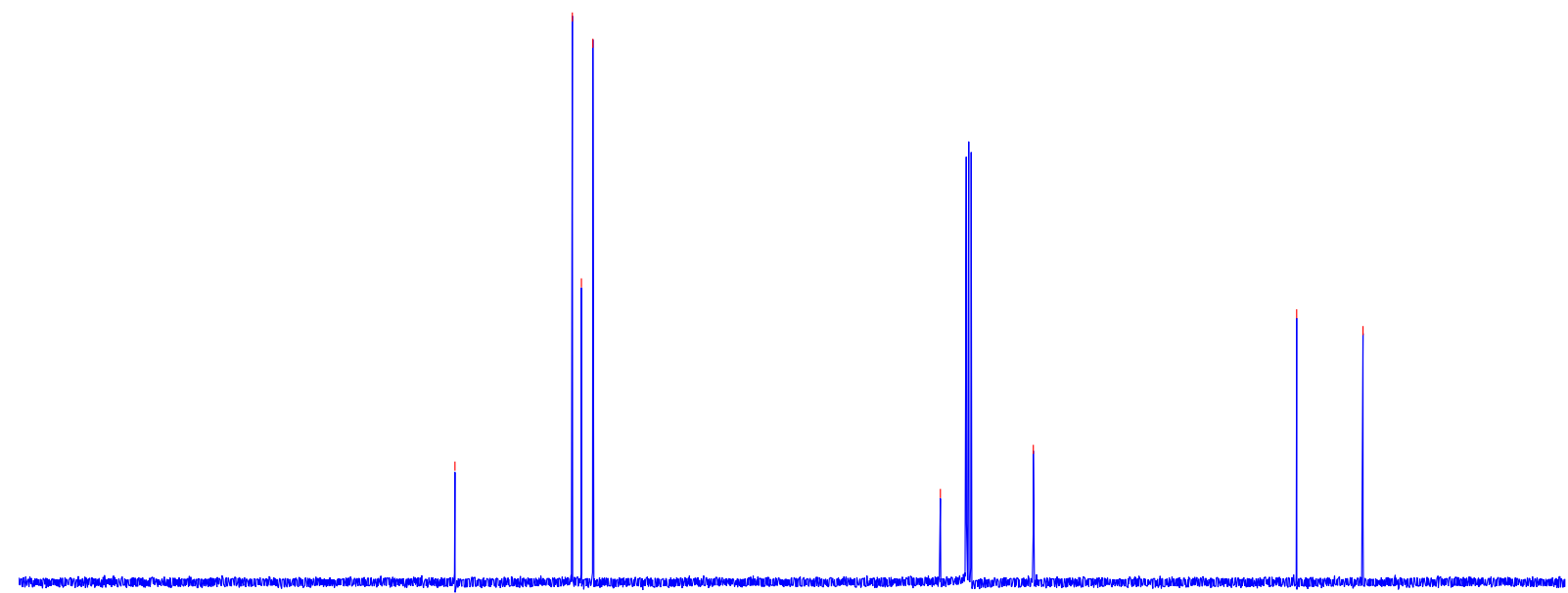

oO 
3g, ${ }^{1} \mathrm{H}$ NMR $\left(400 \mathrm{MHz}, \mathrm{CDCl}_{3}\right)$

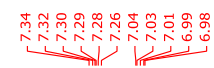

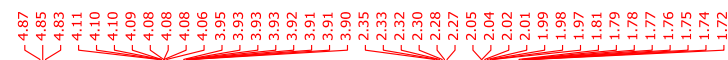<smiles>Fc1ccc(C2CCCO2)cc1</smiles>
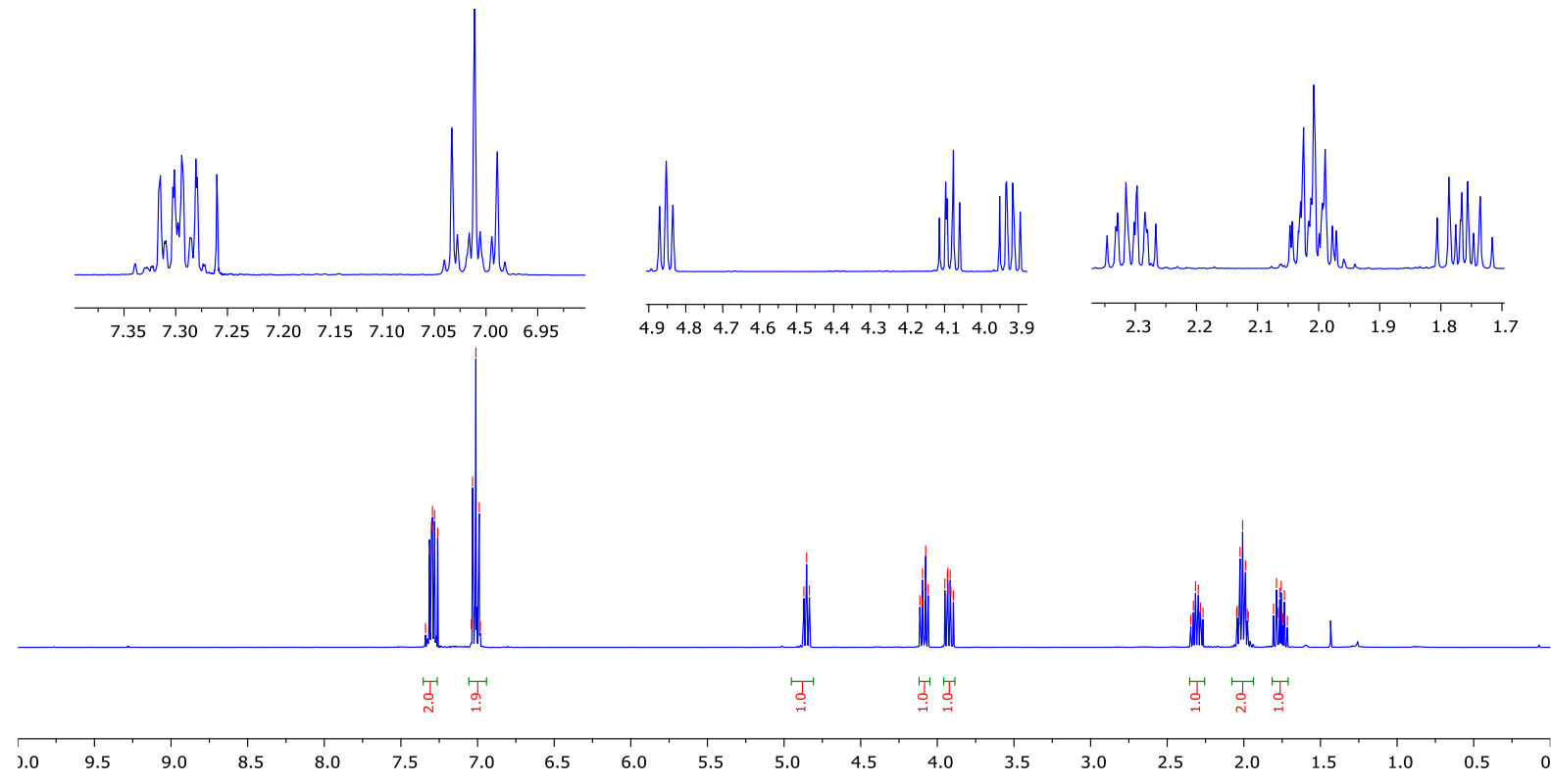

${ }^{13} \mathrm{C}$ NMR (101 MHz, $\left.\mathrm{CDCl}_{3}\right)$

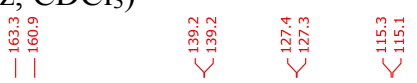

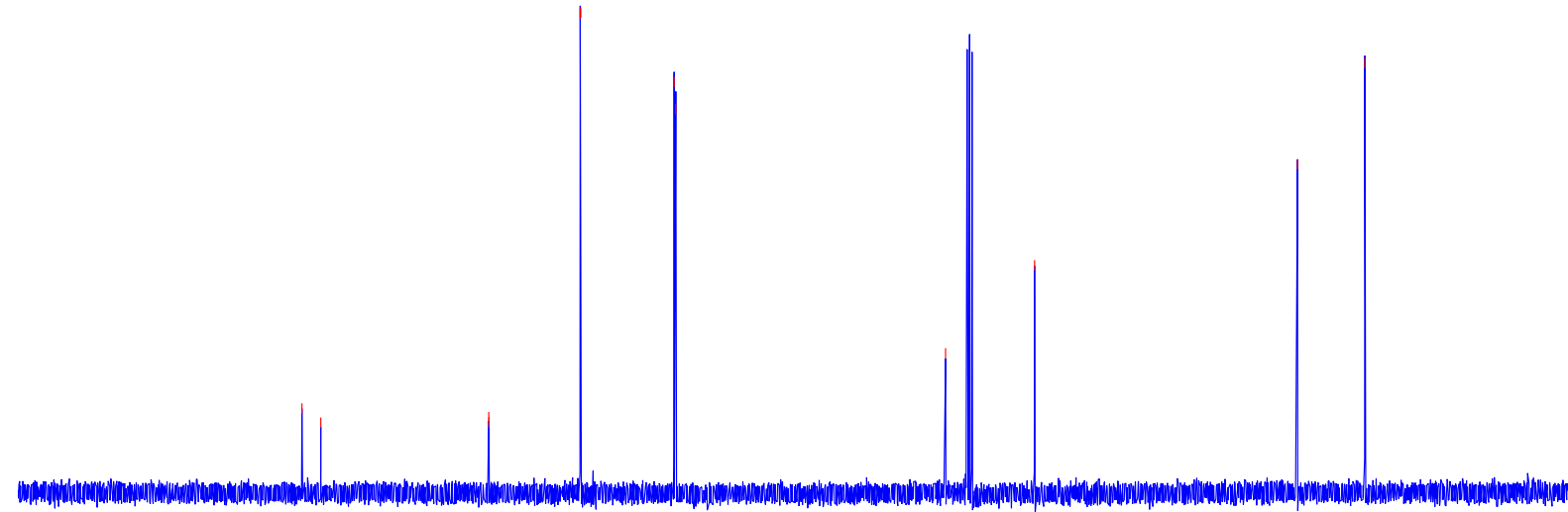

00

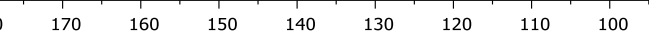
$90 \quad 80 \quad 70$ $60 \quad 50$ $40 \quad 30 \quad 20 \quad 10$ 
$\underline{\mathbf{3 h}},{ }^{1} \mathrm{H}$ NMR $\left(400 \mathrm{MHz}, \mathrm{CDCl}_{3}\right)$

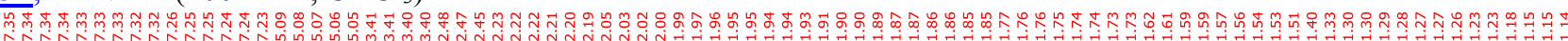<smiles>c1ccc(C2C[C@H]3CCCC[C@H]3O2)cc1</smiles>
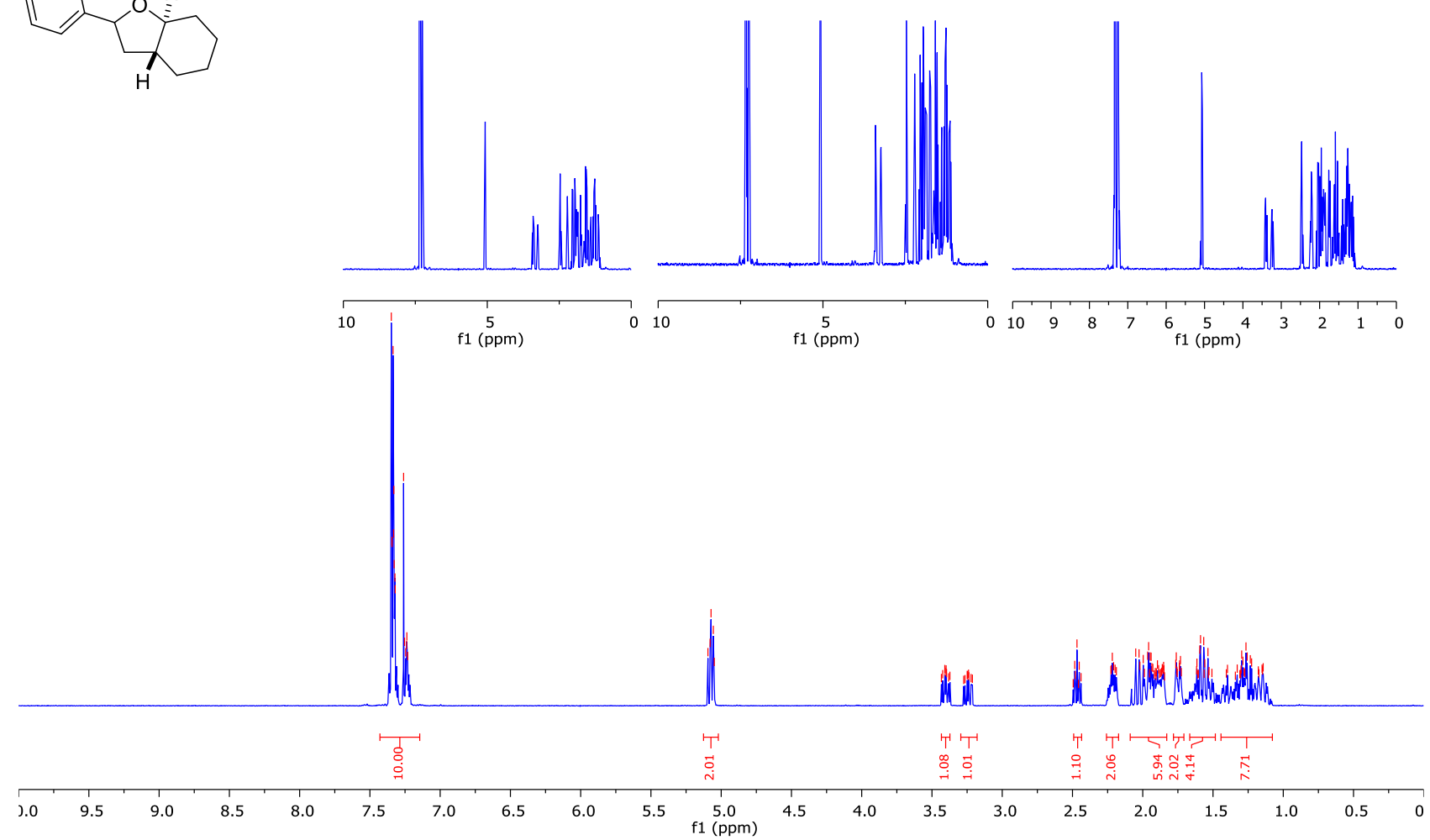

${ }^{13} \mathrm{C}$ NMR (101 MHz, $\left.\mathrm{CDCl}_{3}\right)$

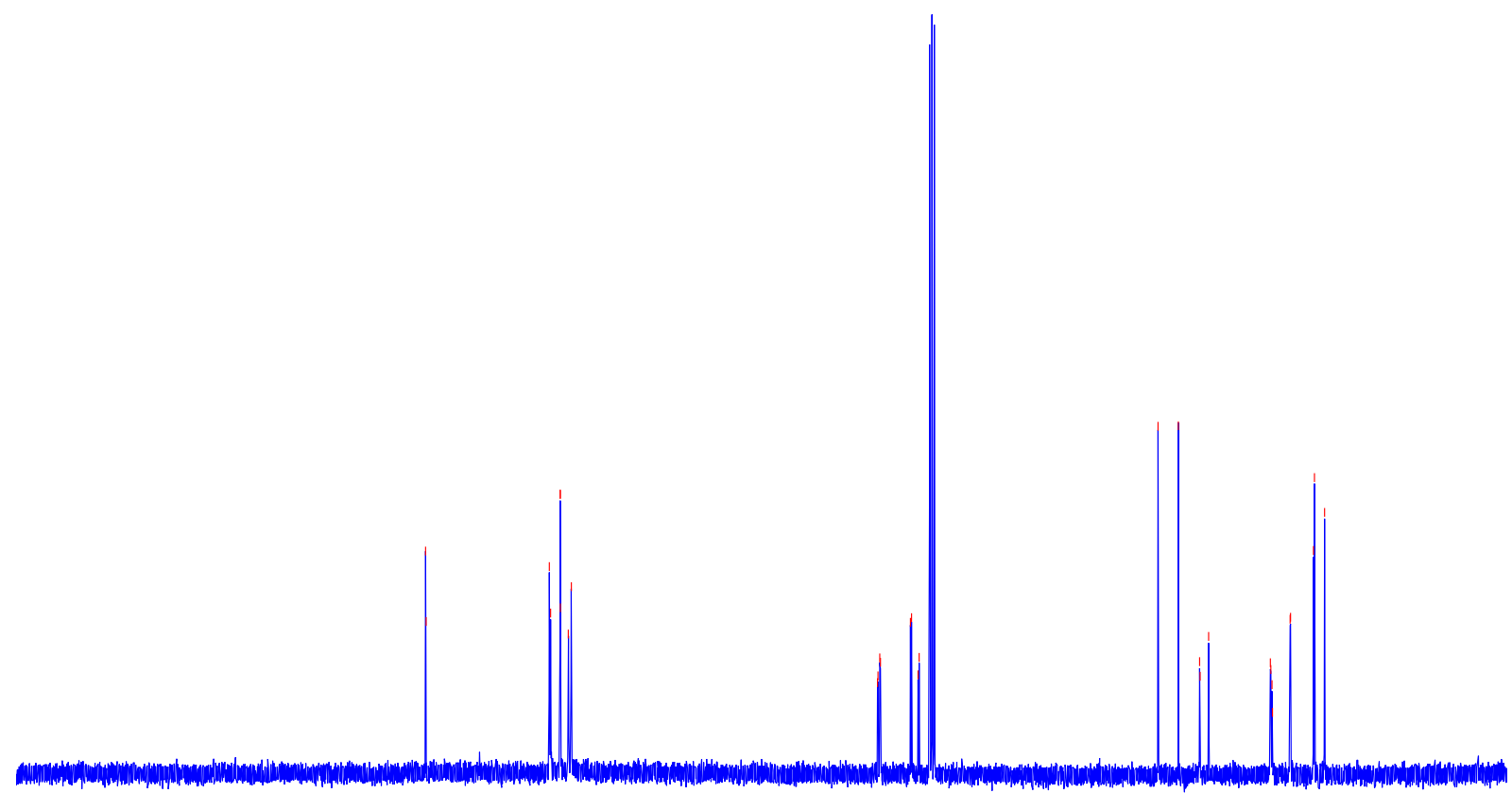

$\begin{array}{lllllllllllllllllllll}00 & 190 & 180 & 170 & 160 & 150 & 140 & 130 & 120 & 110 & \begin{array}{c}100 \\ \mathrm{f} 1(\mathrm{ppm})\end{array} & 90 & 80 & 70 & 60 & 50 & 40 & 30 & 20 & 10 & 1\end{array}$ 
3ii, ${ }^{1} \mathrm{H}$ NMR $\left(400 \mathrm{MHz}, \mathrm{CDCl}_{3}\right)$

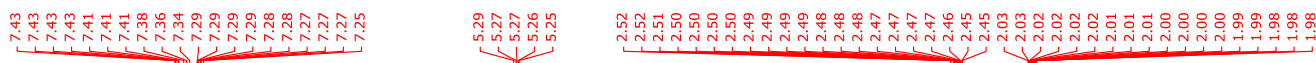
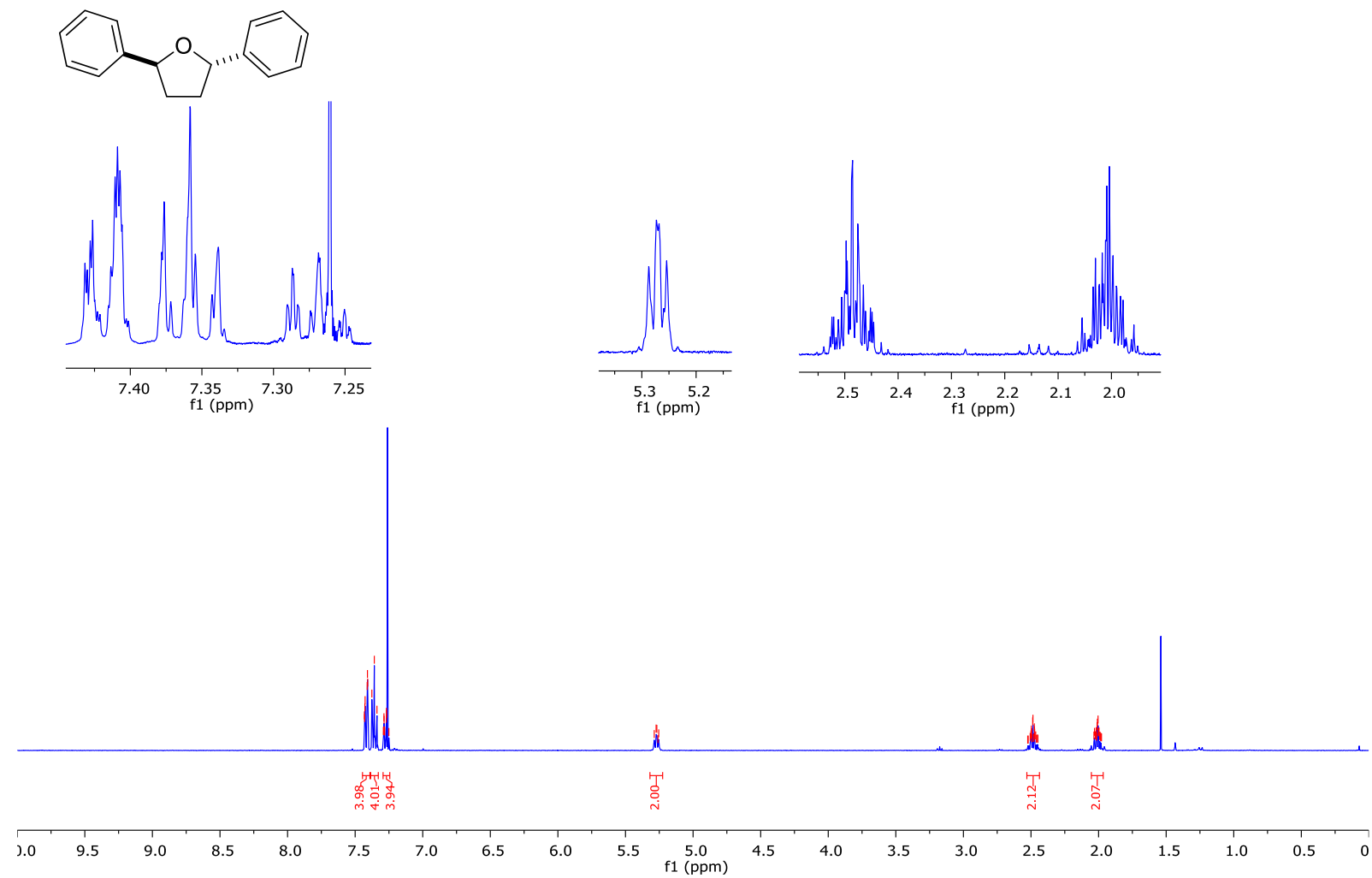

${ }^{13} \mathrm{C}$ NMR (101 MHz, $\left.\mathrm{CDCl}_{3}\right)$

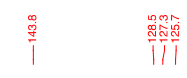

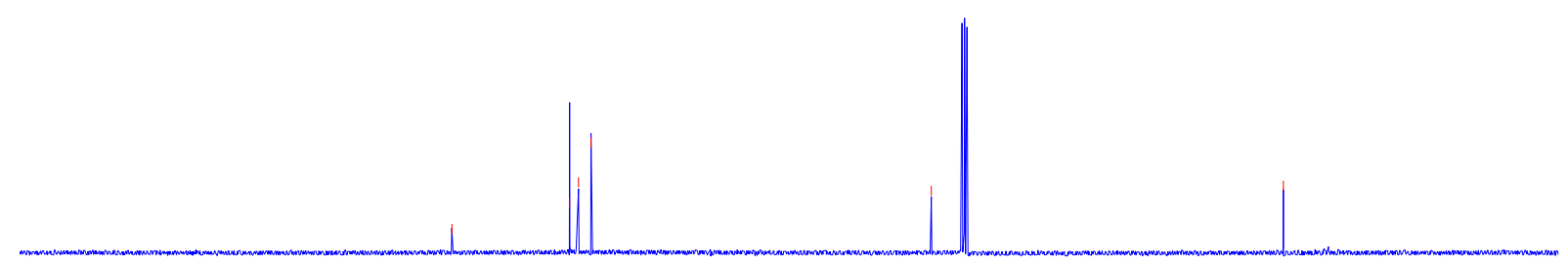

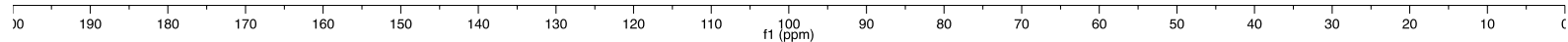


$\underline{\text { 3i, }}{ }^{1} \mathrm{H}$ NMR $\left(400 \mathrm{MHz}, \mathrm{CDCl}_{3}\right)$

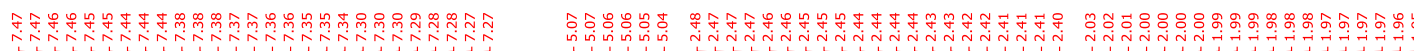

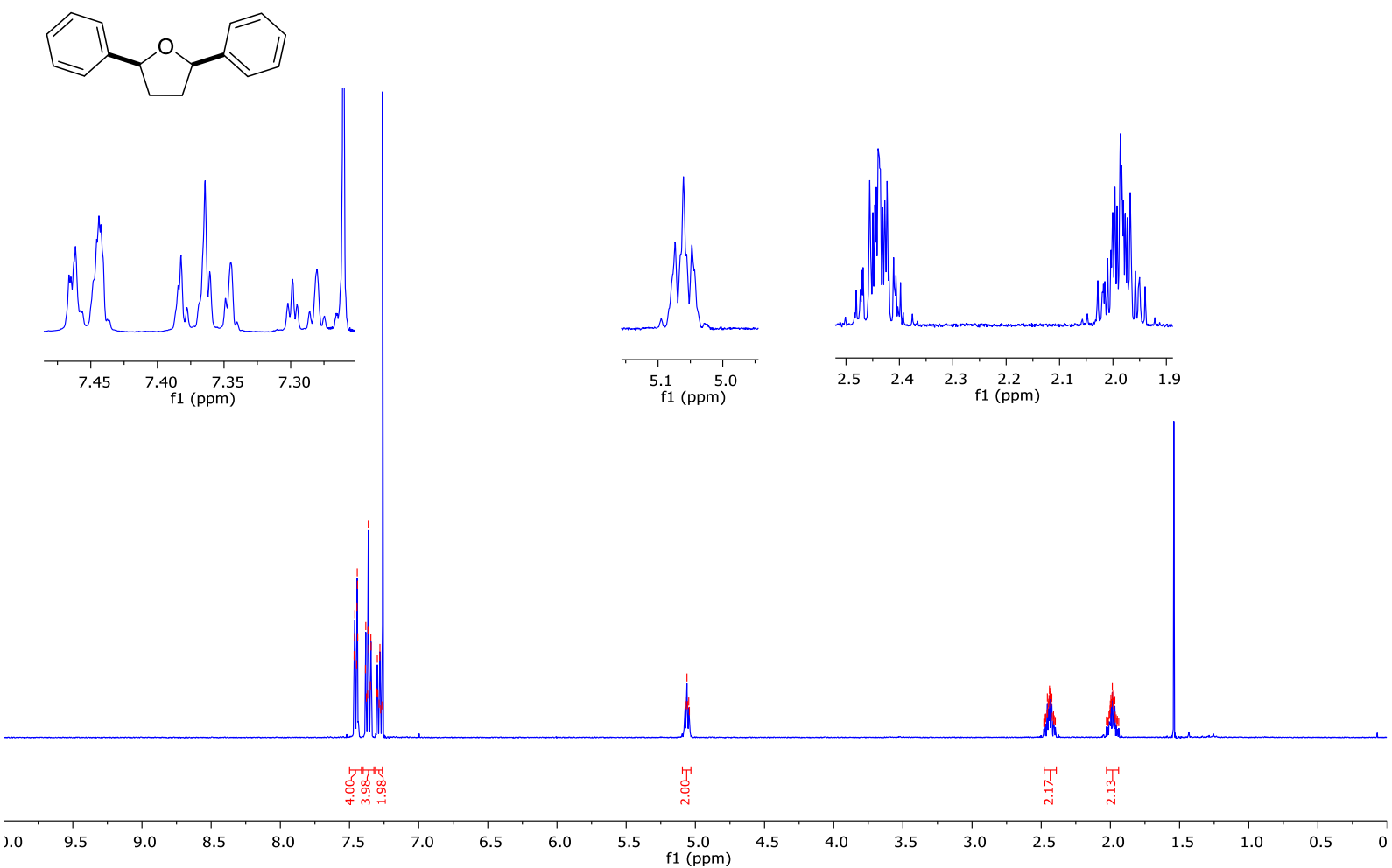

${ }^{13} \mathrm{C}$ NMR $\left(101 \mathrm{MHz}, \mathrm{CDCl}_{3}\right)$

踔

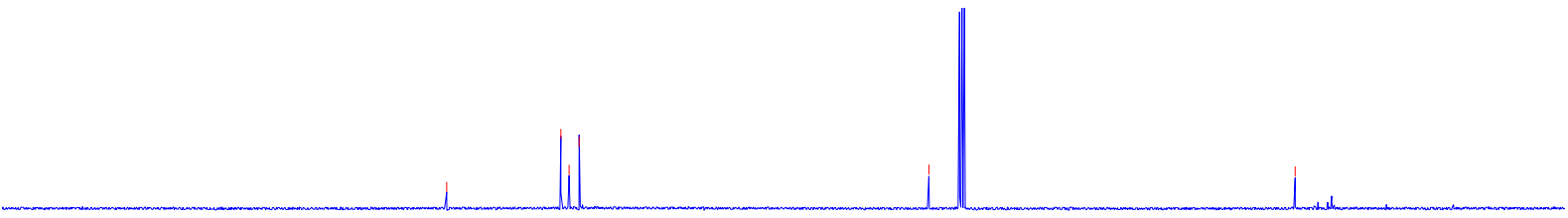

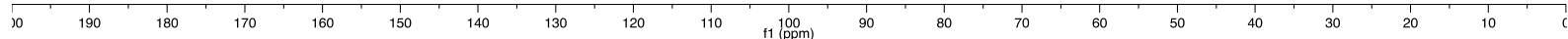


3j, ${ }^{1} \mathrm{H}$ NMR (400 MHz, $\left.\mathrm{CDCl}_{3}\right)$

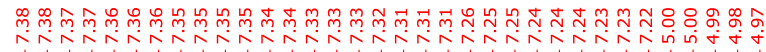

ભ̊

12
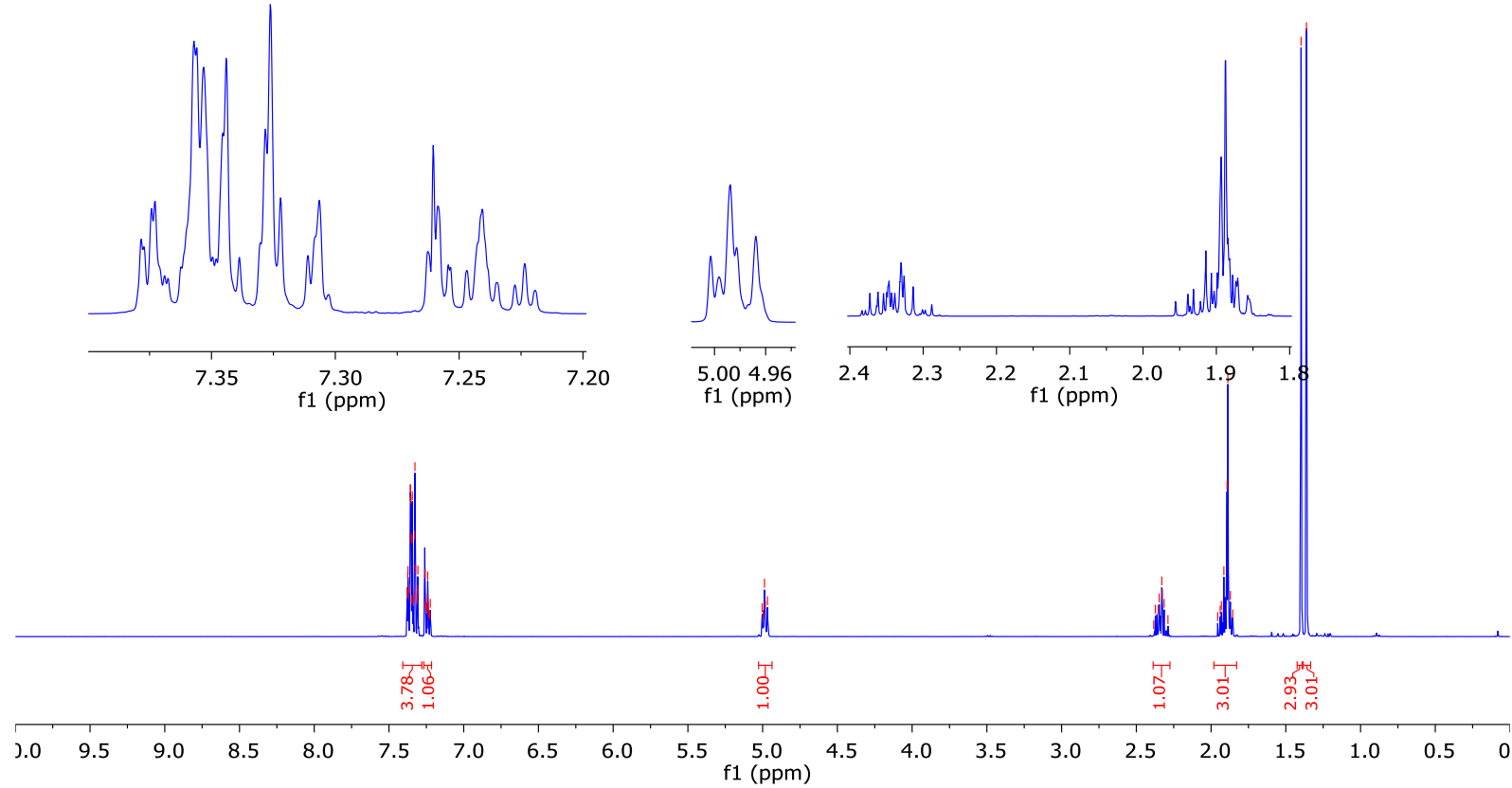

${ }^{13} \mathrm{C}$ NMR (101 MHz, $\left.\mathrm{CDCl}_{3}\right)$

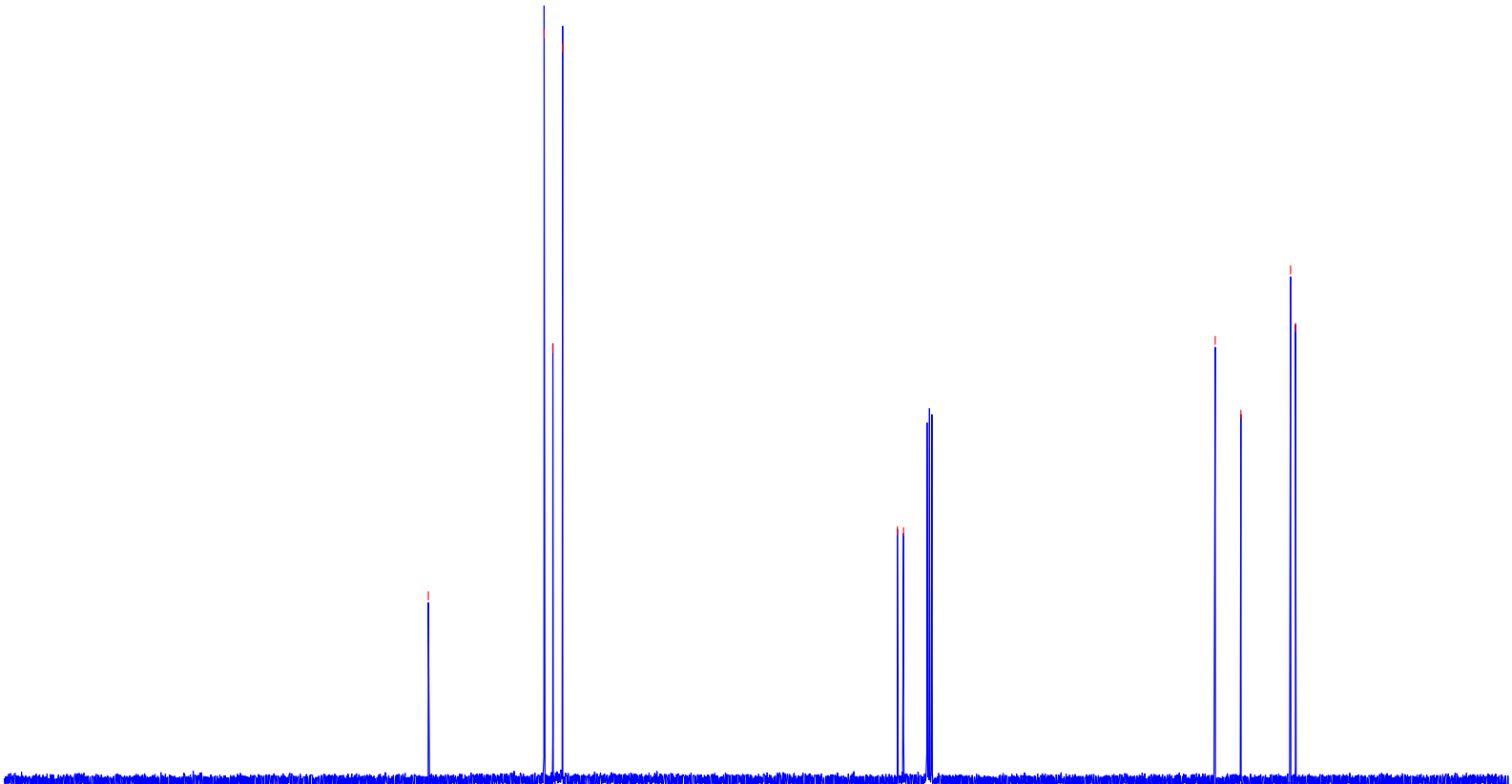


$\underline{\mathbf{3 k}},{ }^{1} \mathrm{H}$ NMR (400 MHz, $\mathrm{CDCl}_{3}$ )

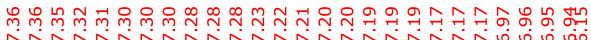
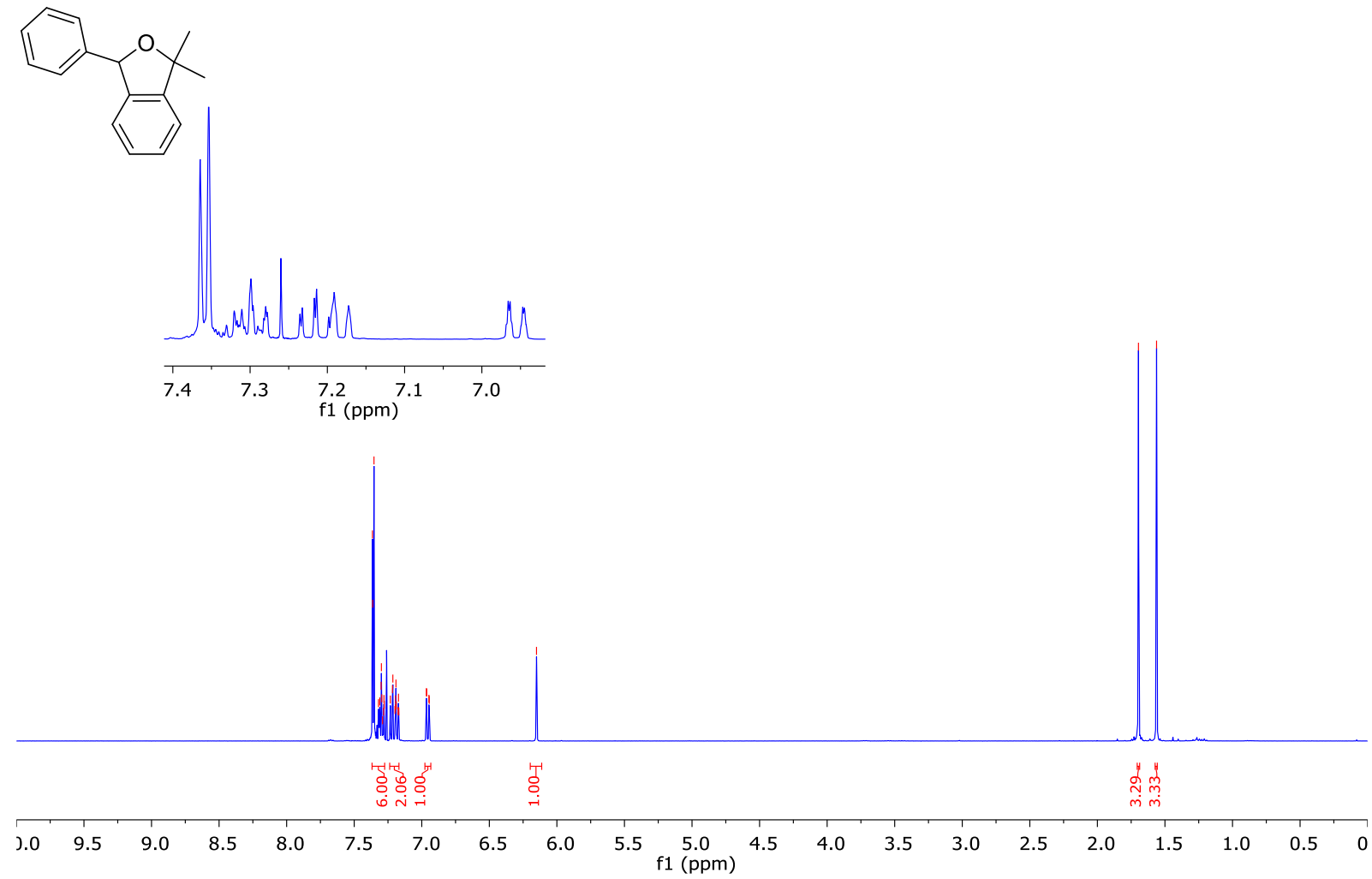

${ }^{13} \mathrm{C}$ NMR (101 MHz, $\left.\mathrm{CDCl}_{3}\right)$

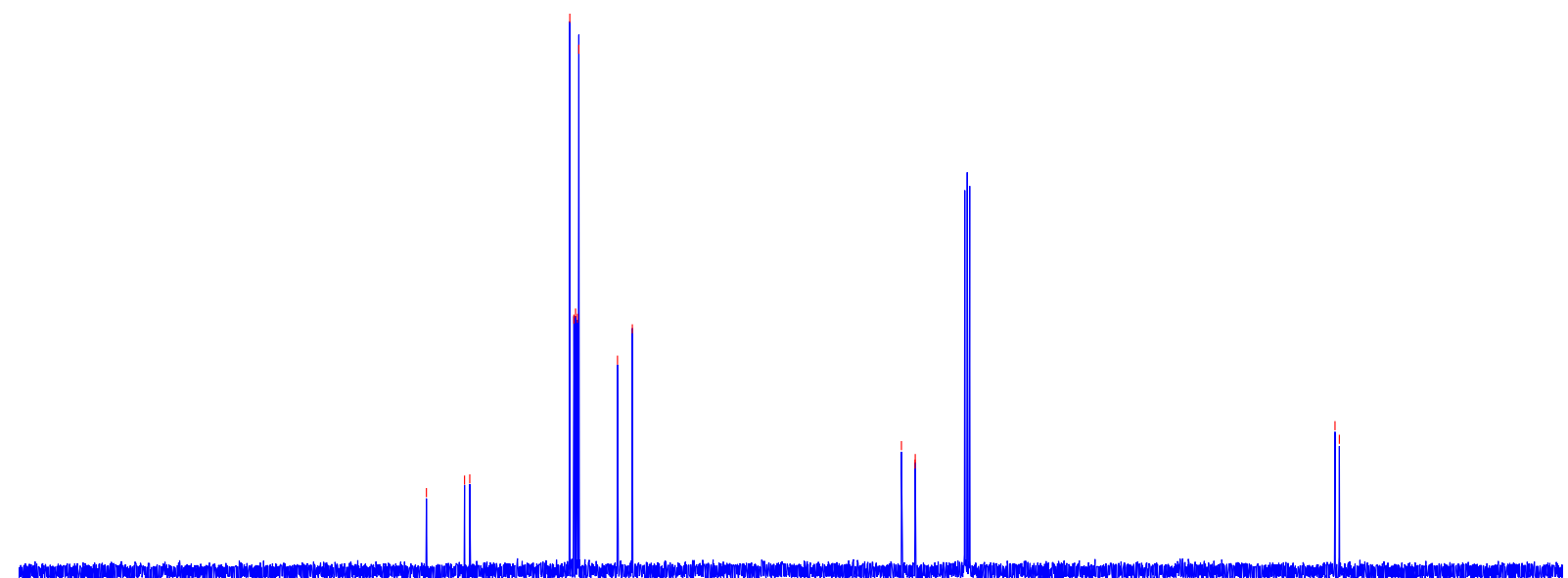

$00 \quad 190 \quad 180$

$\begin{array}{lllllll}170 & 160 & 150 & 140 & 130 & 120\end{array}$

$10 \underset{f 1}{100}(\mathrm{ppm})$ 
31, ${ }^{1} \mathrm{H}$ NMR $\left(400 \mathrm{MHz}, \mathrm{CDCl}_{3}\right)$

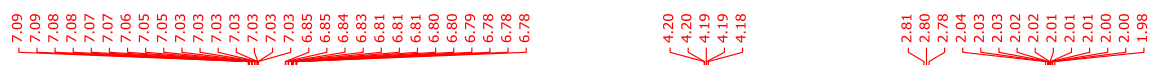<smiles>c1ccc2c(c1)CCCO2</smiles>
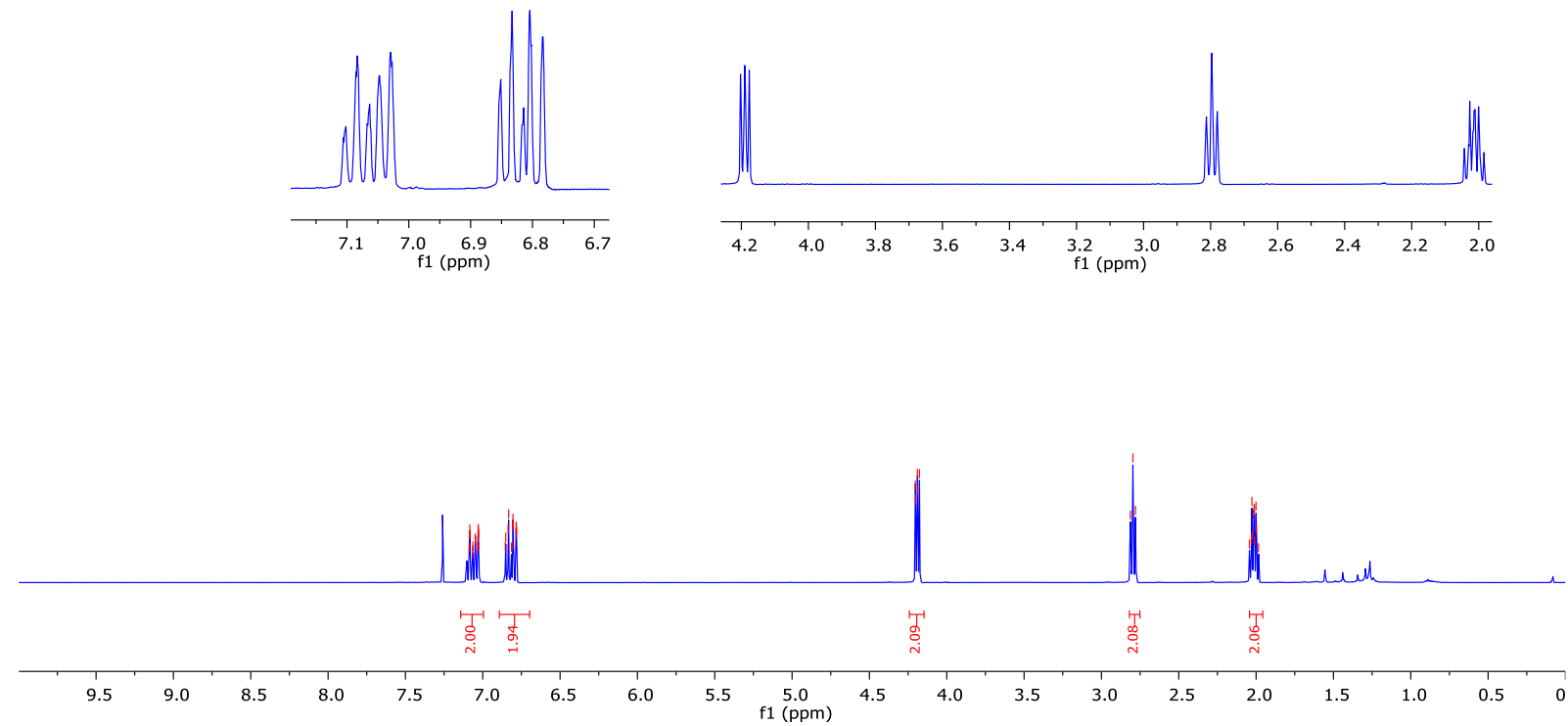

${ }^{13} \mathrm{C}$ NMR (101 MHz, $\left.\mathrm{CDCl}_{3}\right)$

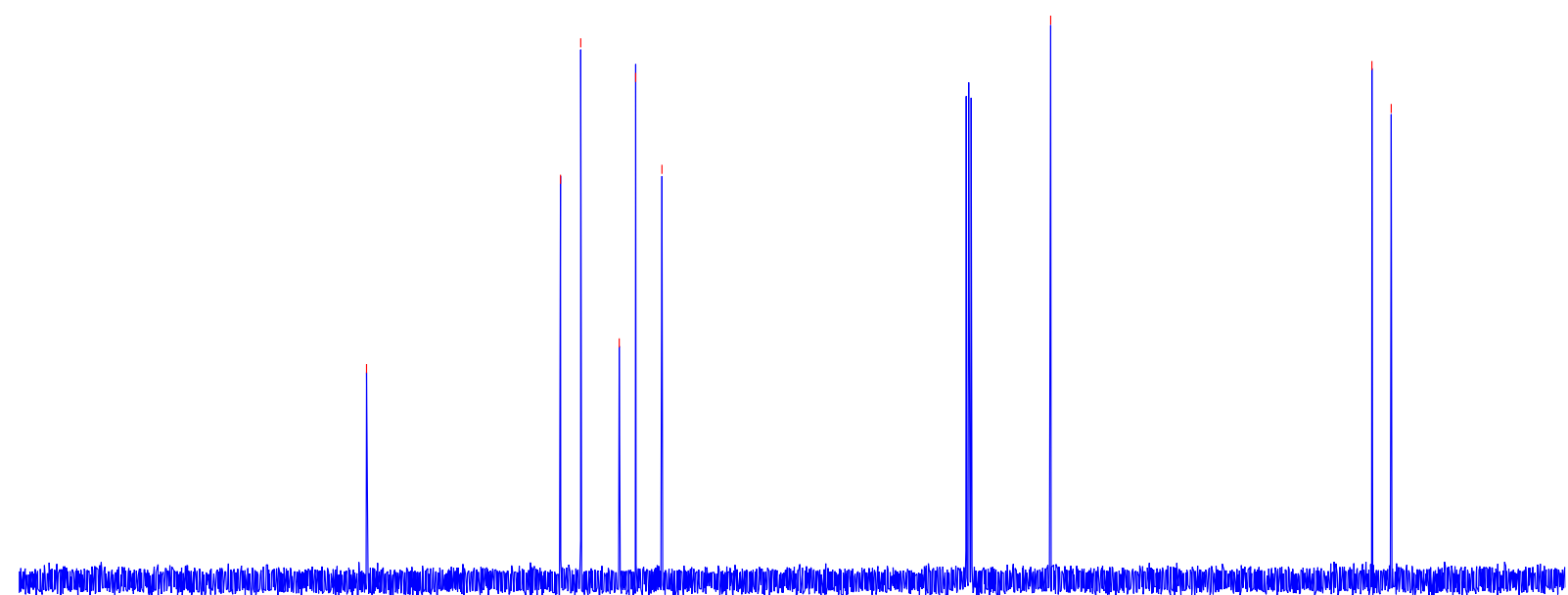


$\underline{\mathbf{3 m}},{ }^{1} \mathrm{H}$ NMR $\left(400 \mathrm{MHz}, \mathrm{CDCl}_{3}\right)$<smiles>COc1cc2c(c(OC)c1OC)OCCC2</smiles>

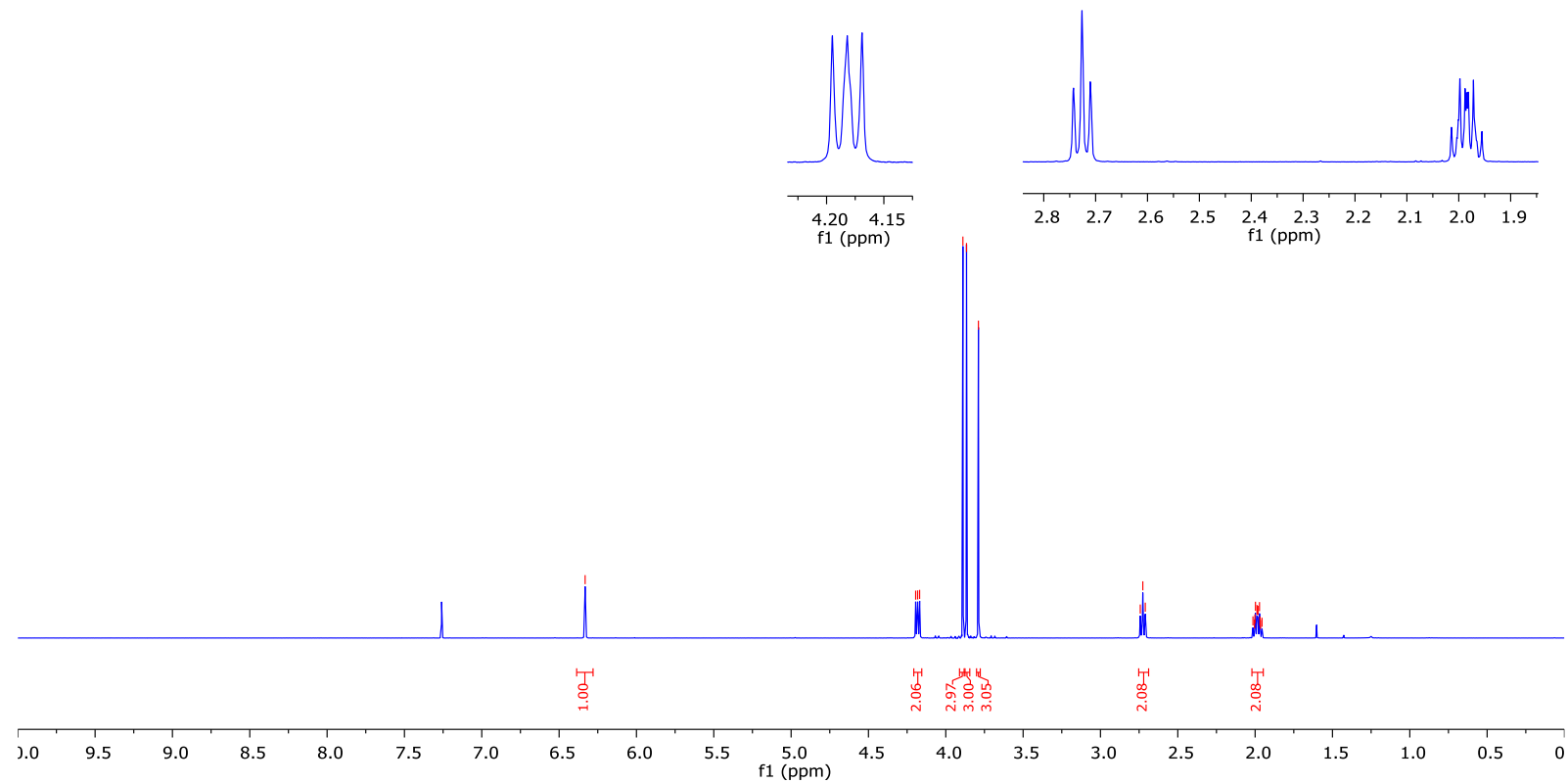

${ }^{13} \mathrm{C}$ NMR (101 MHz, $\left.\mathrm{CDCl}_{3}\right)$

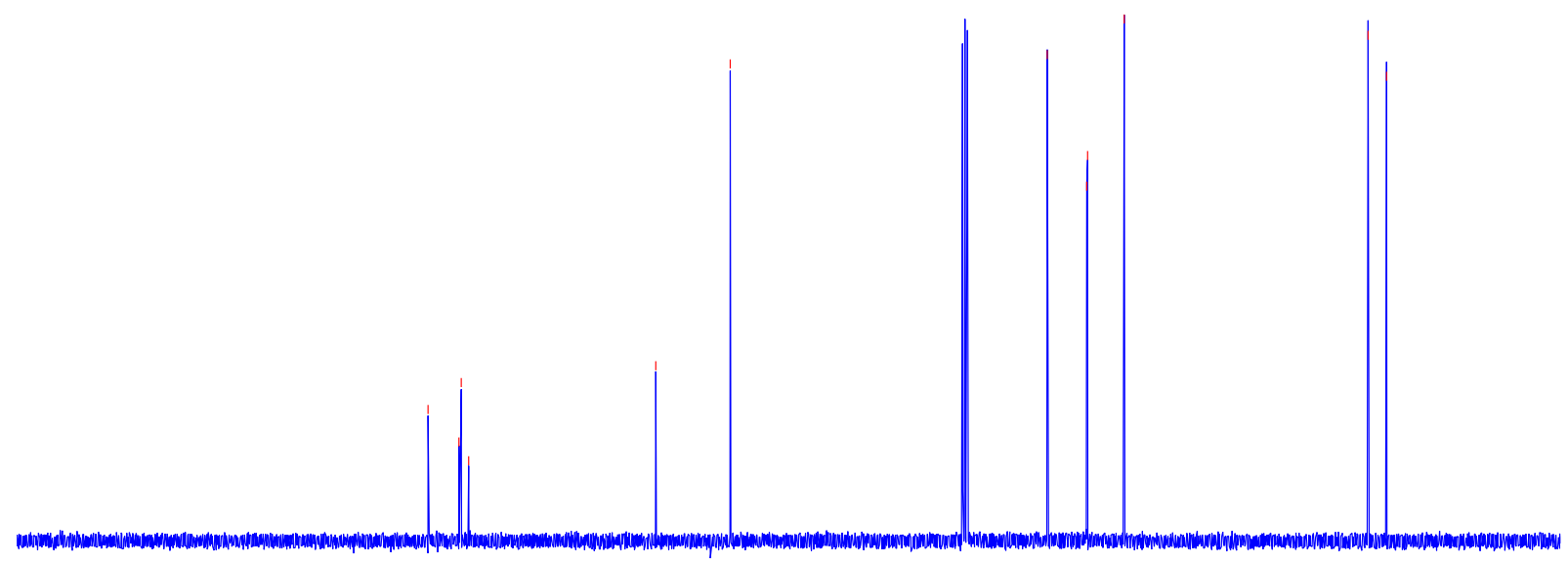

Do 
3n, ${ }^{1} \mathrm{H}$ NMR $\left(400 \mathrm{MHz}, \mathrm{CDCl}_{3}\right)$
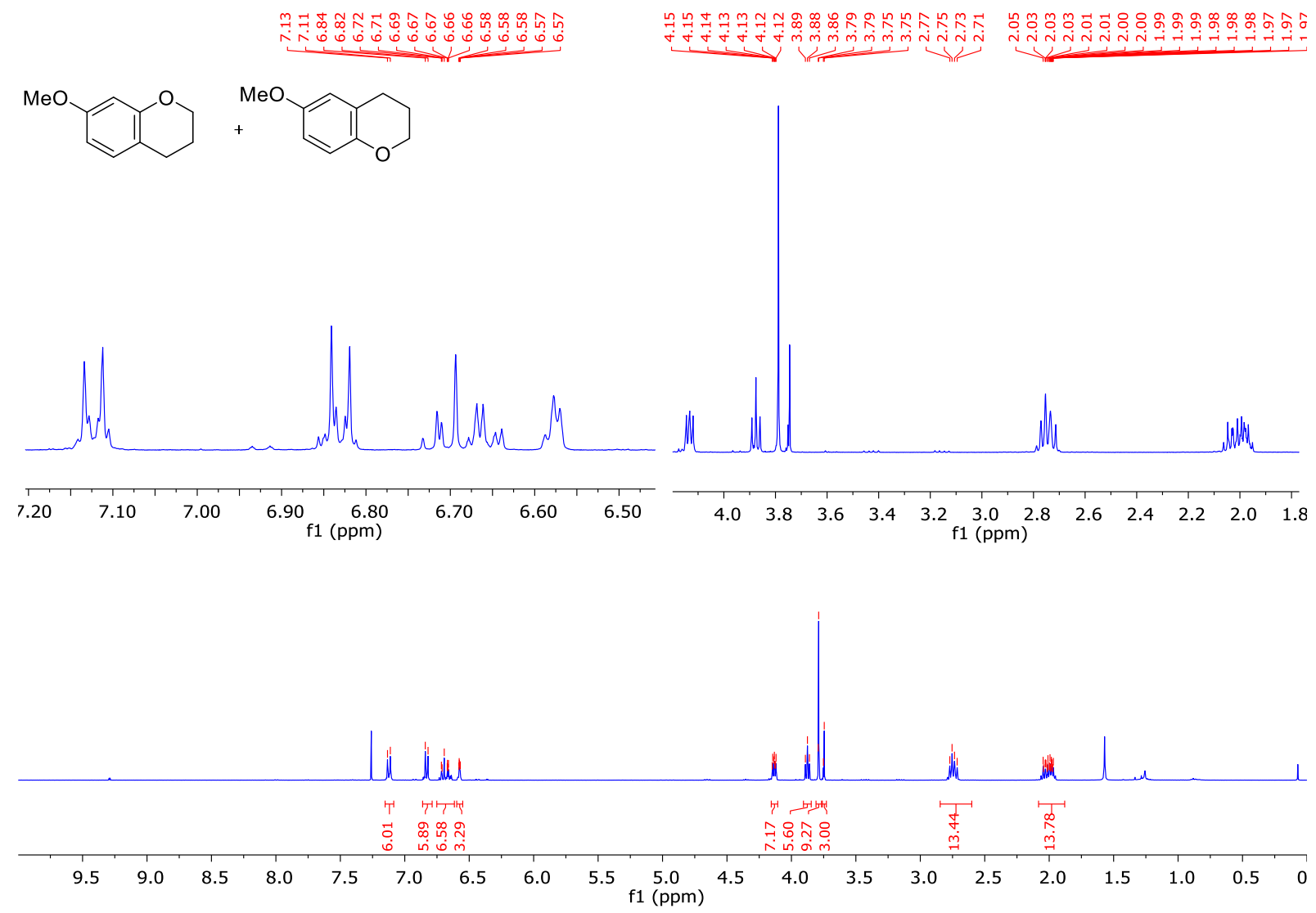

${ }^{13} \mathrm{C}$ NMR $\left(101 \mathrm{MHz}, \mathrm{CDCl}_{3}\right)$
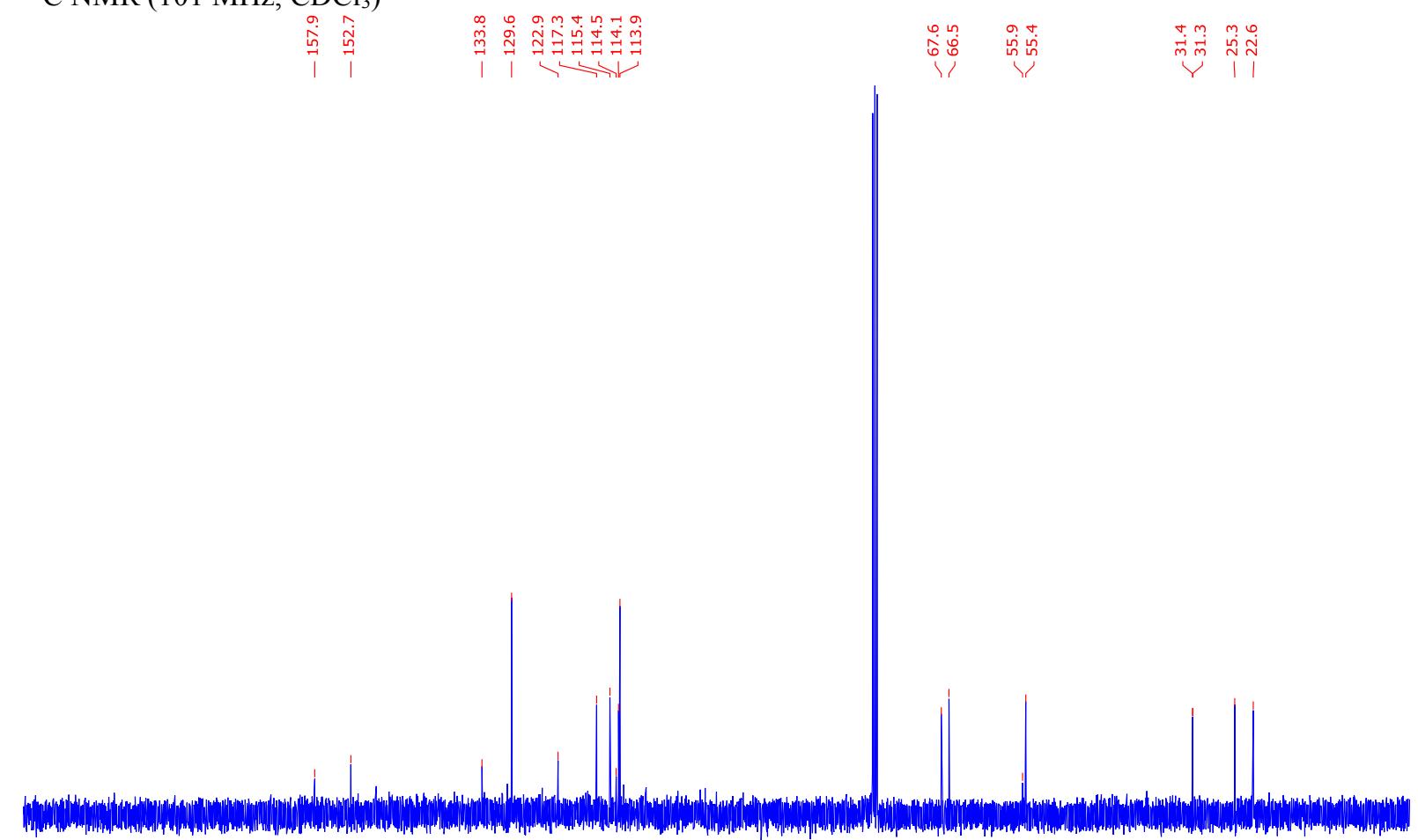

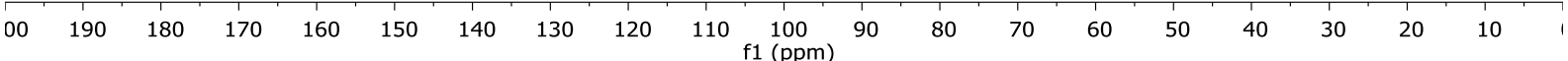


3p,${ }^{1} \mathrm{H}$ NMR $\left(400 \mathrm{MHz}, \mathrm{CDCl}_{3}\right)$

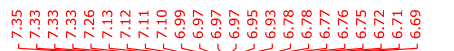

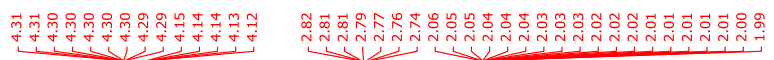

(1)
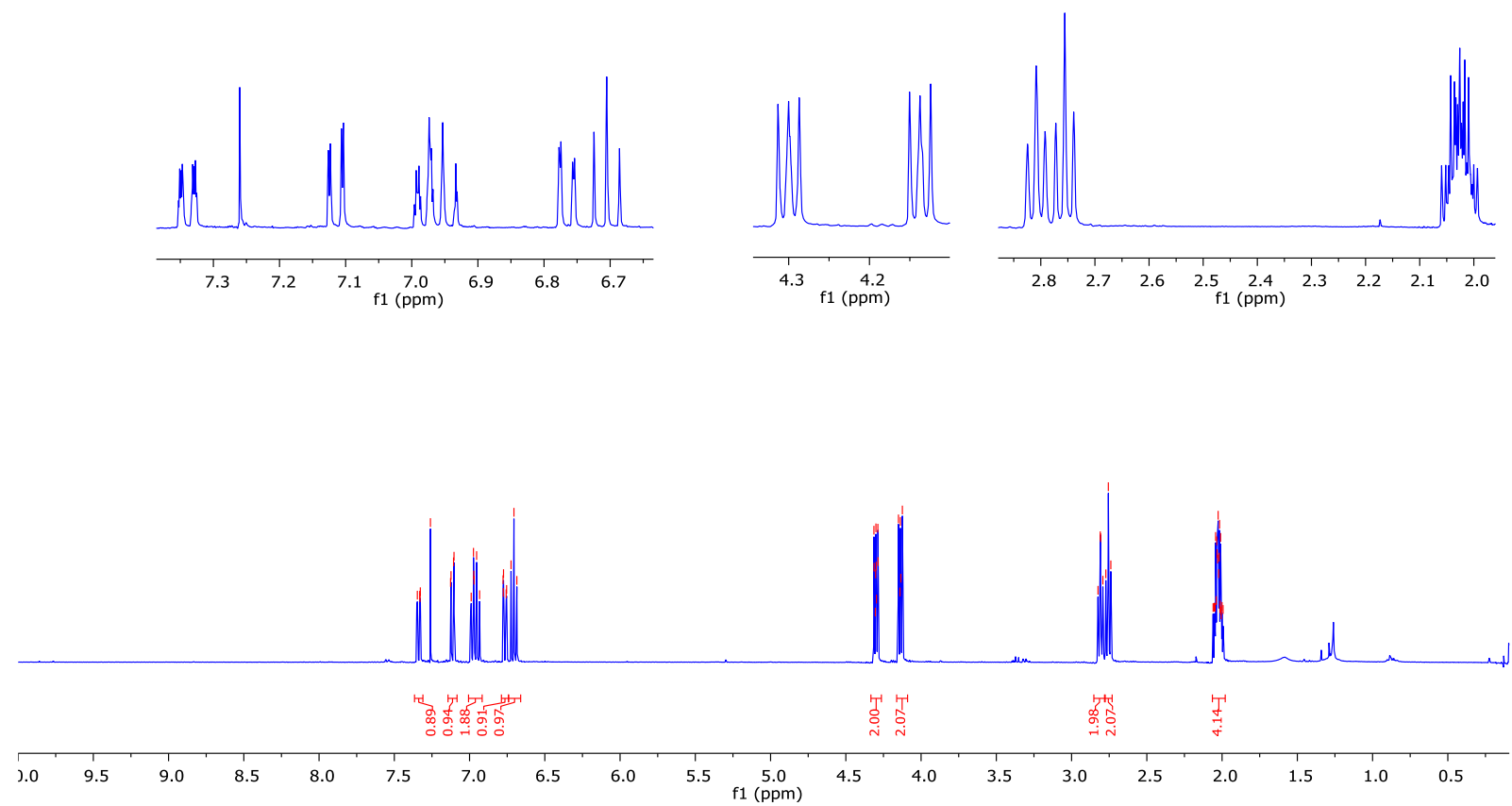

${ }^{13} \mathrm{C}$ NMR (101 MHz, $\left.\mathrm{CDCl}_{3}\right)$
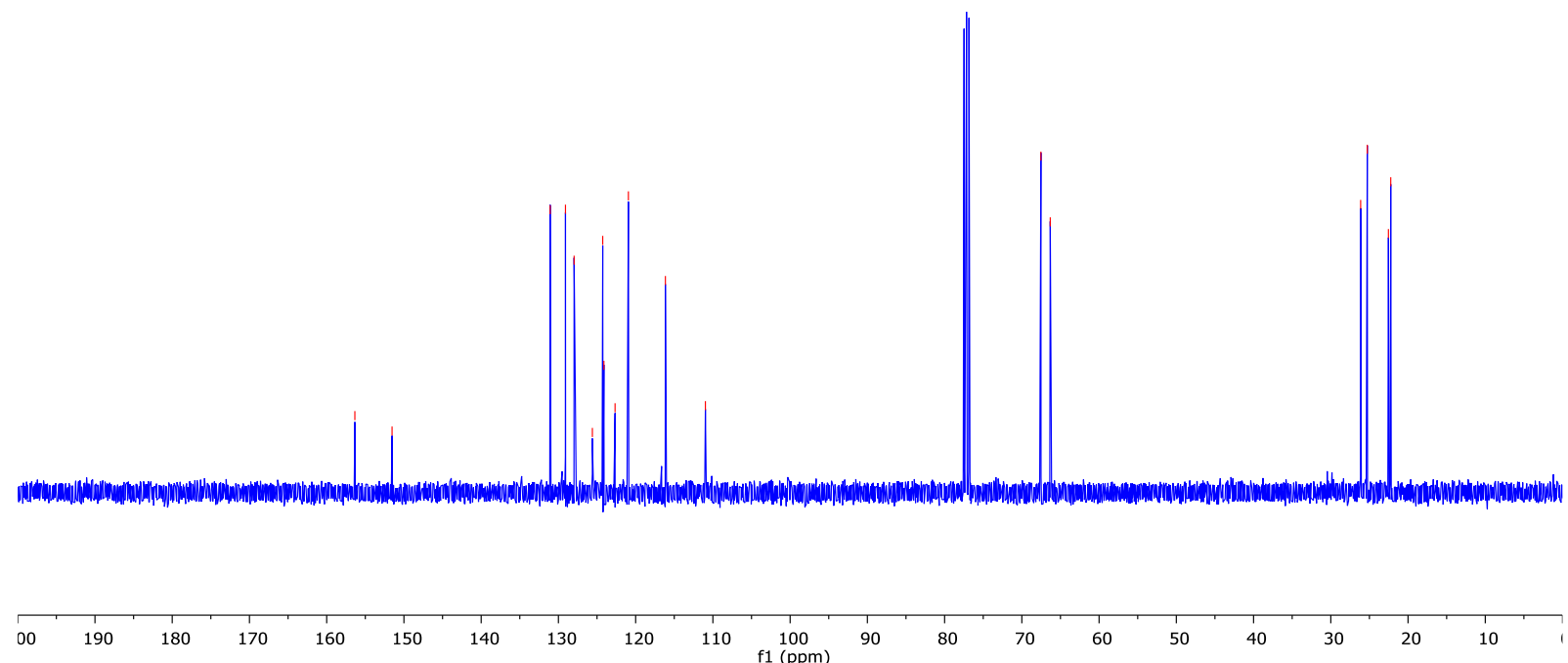

70

50 
3q, ${ }^{1} \mathrm{H}$ NMR $\left(400 \mathrm{MHz}, \mathrm{CDCl}_{3}\right)$

$\underbrace{.0}$

$\mathrm{Cl}$<smiles></smiles>
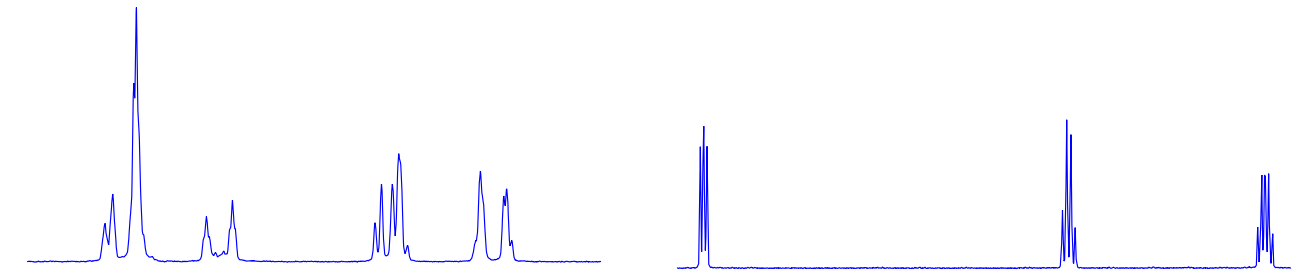

$\begin{array}{rrrrrrrrrr}7.10 & 7.05 & 7.00 & 6.95 & 6.90 & 6.85 & 6.80 & 6.75 & 6.70 & 6.65 \\ \text { f1 (ppm) } & & & & & & & \end{array}$
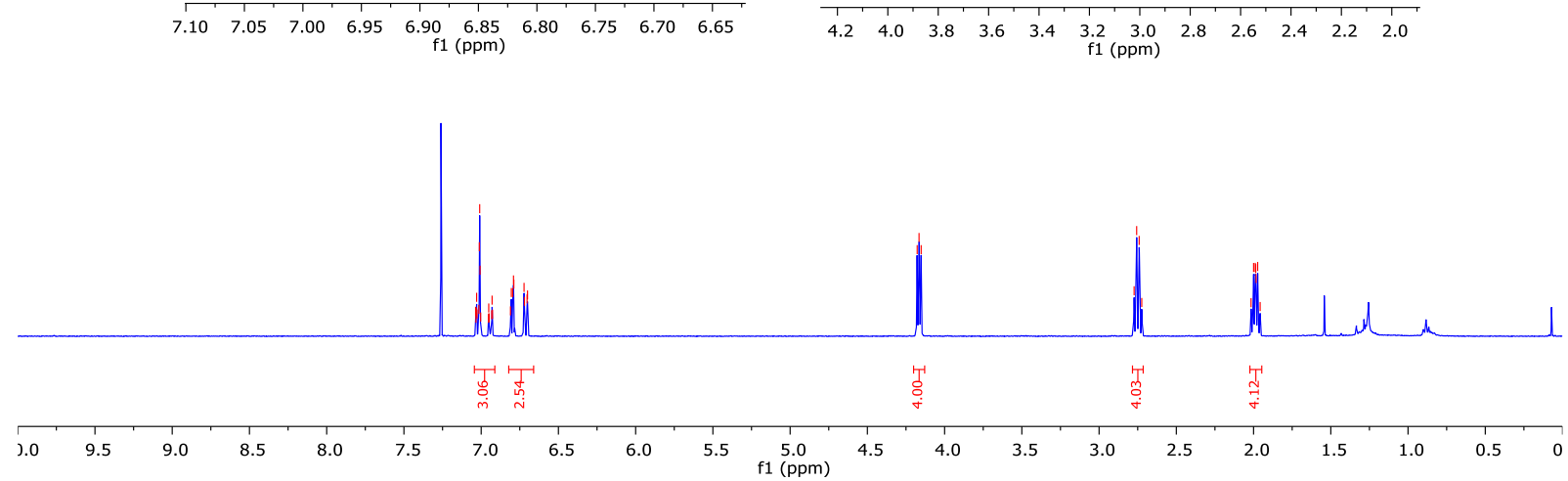

${ }^{13} \mathrm{C}$ NMR (101 MHz, $\left.\mathrm{CDCl}_{3}\right)$

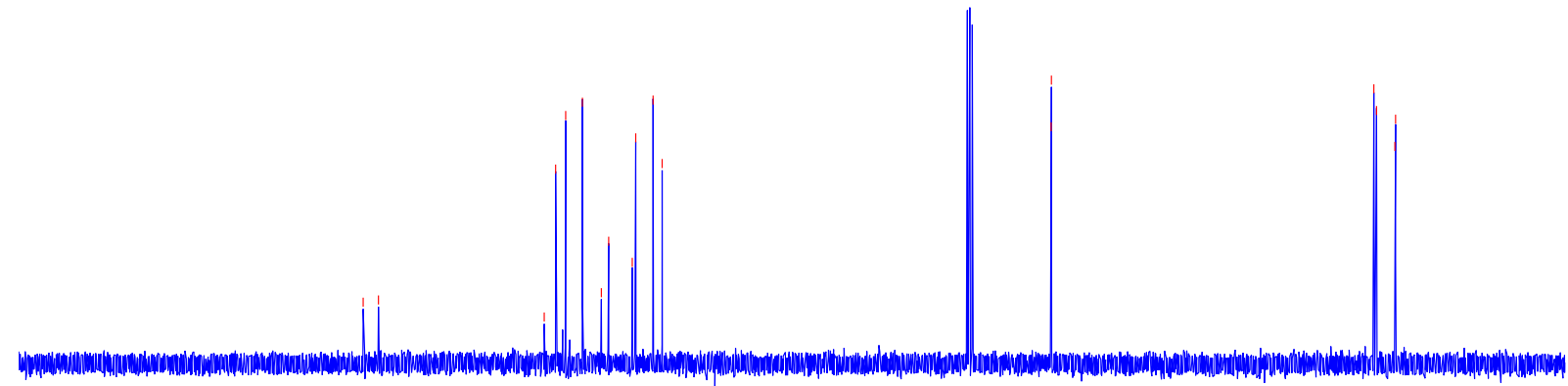
190
180
170
160
150
13
120

$\begin{array}{cc}110 & 100 \\ \mathrm{f} 1(\mathrm{ppm})\end{array}$

$90 \quad 80$

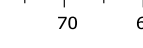

$50 \quad 40$ 
<smiles>c1ccc2c(c1)CC1CCCCC1O2</smiles>
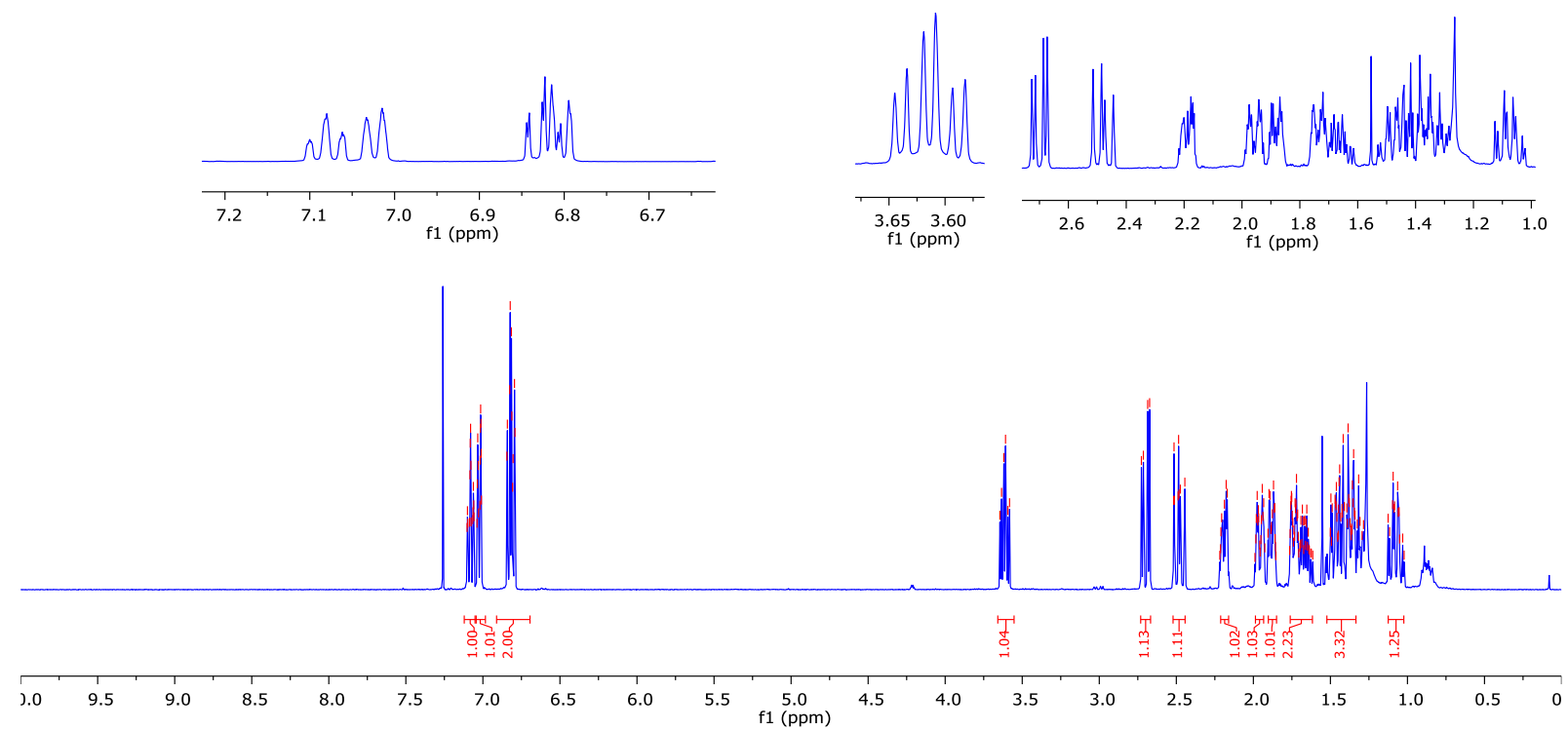

${ }^{13} \mathrm{C}$ NMR (101 MHz, $\mathrm{CDCl}_{3}$ )

1

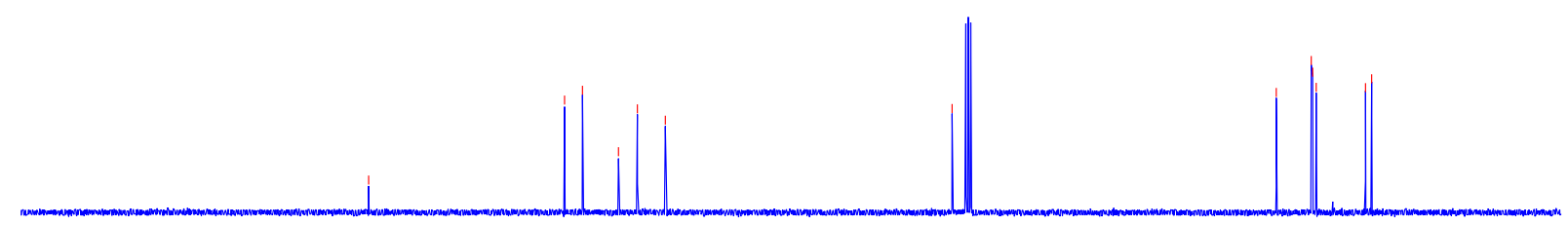

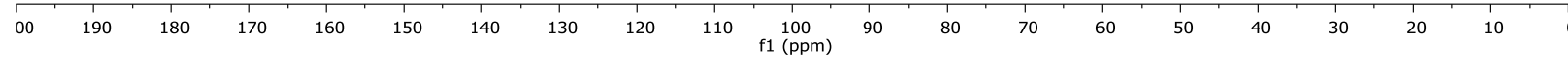


$\underline{\mathbf{3 s}},{ }^{1} \mathrm{H}$ NMR (400 MHz, $\left.\mathrm{CDCl}_{3}\right)$

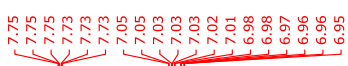<smiles>CC1(C)Oc2ccccc2-c2ccccc21</smiles>
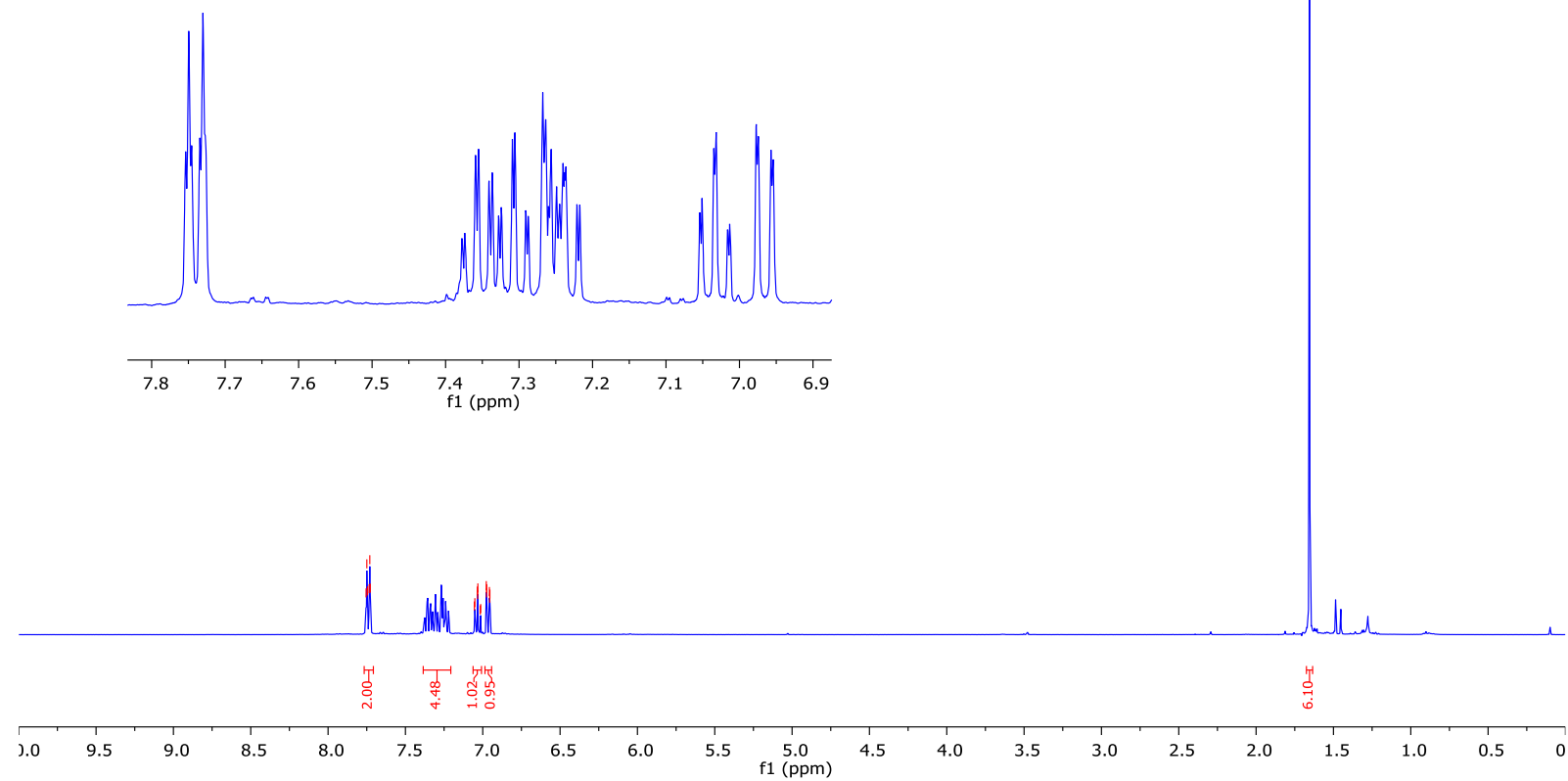

${ }^{13} \mathrm{C}$ NMR (101 MHz, $\mathrm{CDCl}_{3}$ )

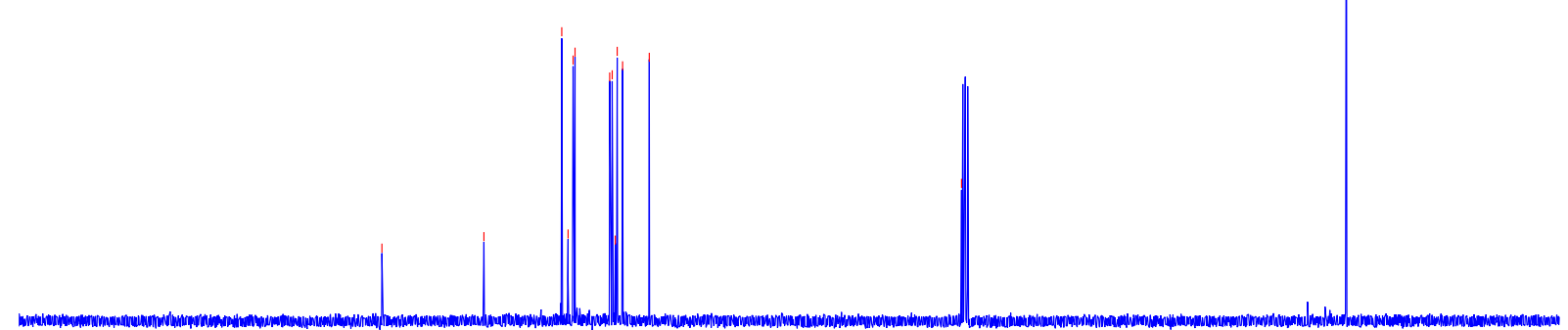

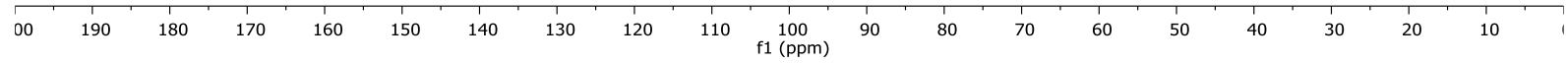




\section{3t, ${ }^{1} \mathrm{H}$ NMR (400 MHz, $\left.\mathrm{CDCl}_{3}\right)$}

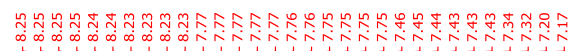

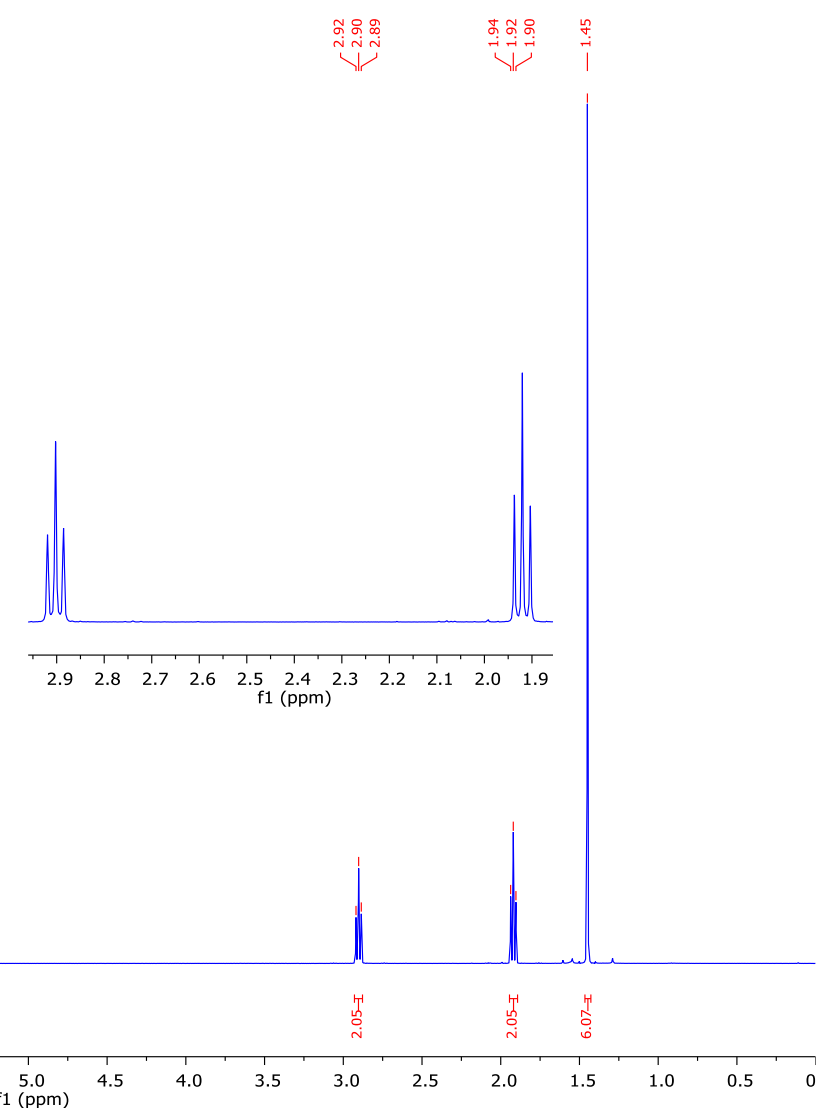

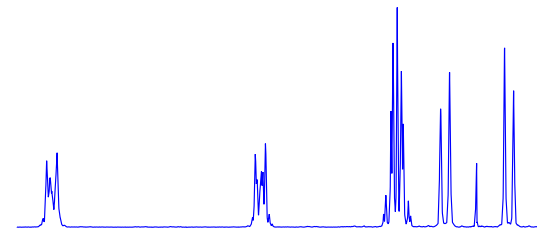

$\begin{array}{lllllllllllllllll}8.3 & 8.2 & 8.1 & 8.0 & 7.9 & 7.8 & 7.7 & 7.6 & 7.5 & 7.4 & 7.3 & 7.2\end{array}$<smiles>CC1(C)CCc2ccc3ccccc3c2O1</smiles>

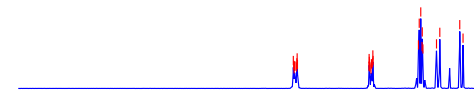

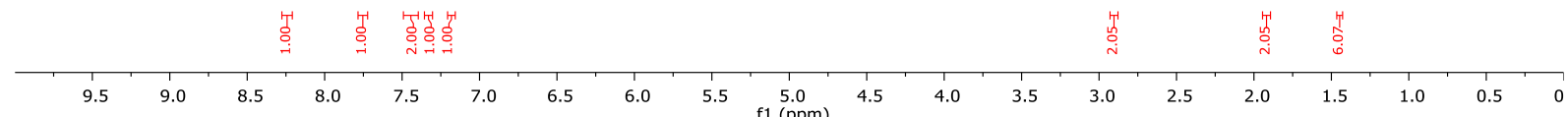

${ }^{13} \mathrm{C}$ NMR (101 MHz, $\left.\mathrm{CDCl}_{3}\right)$

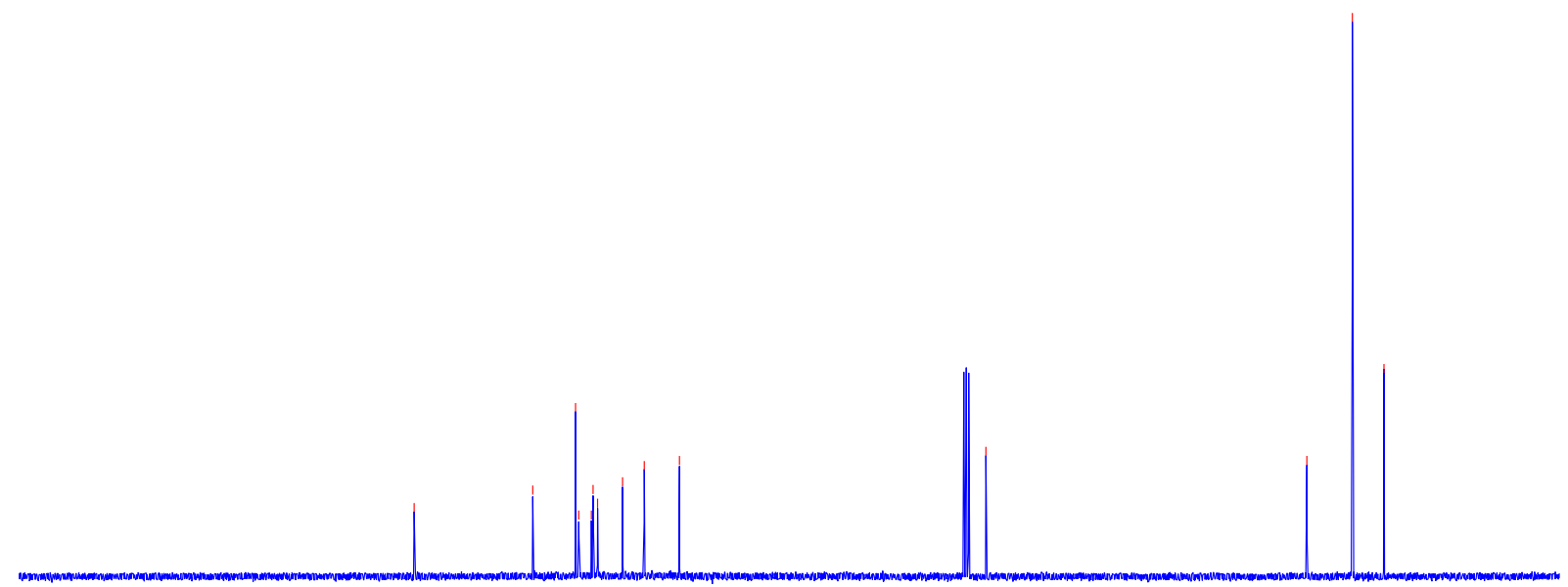

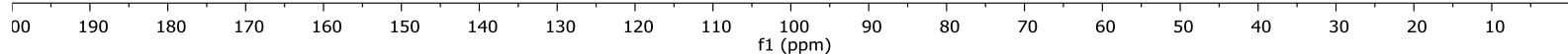


$\underline{\text { 3t', }}{ }^{1} \mathrm{H}$ NMR $\left(400 \mathrm{MHz}, \mathrm{CDCl}_{3}\right)$

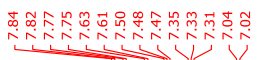

$N_{0}$
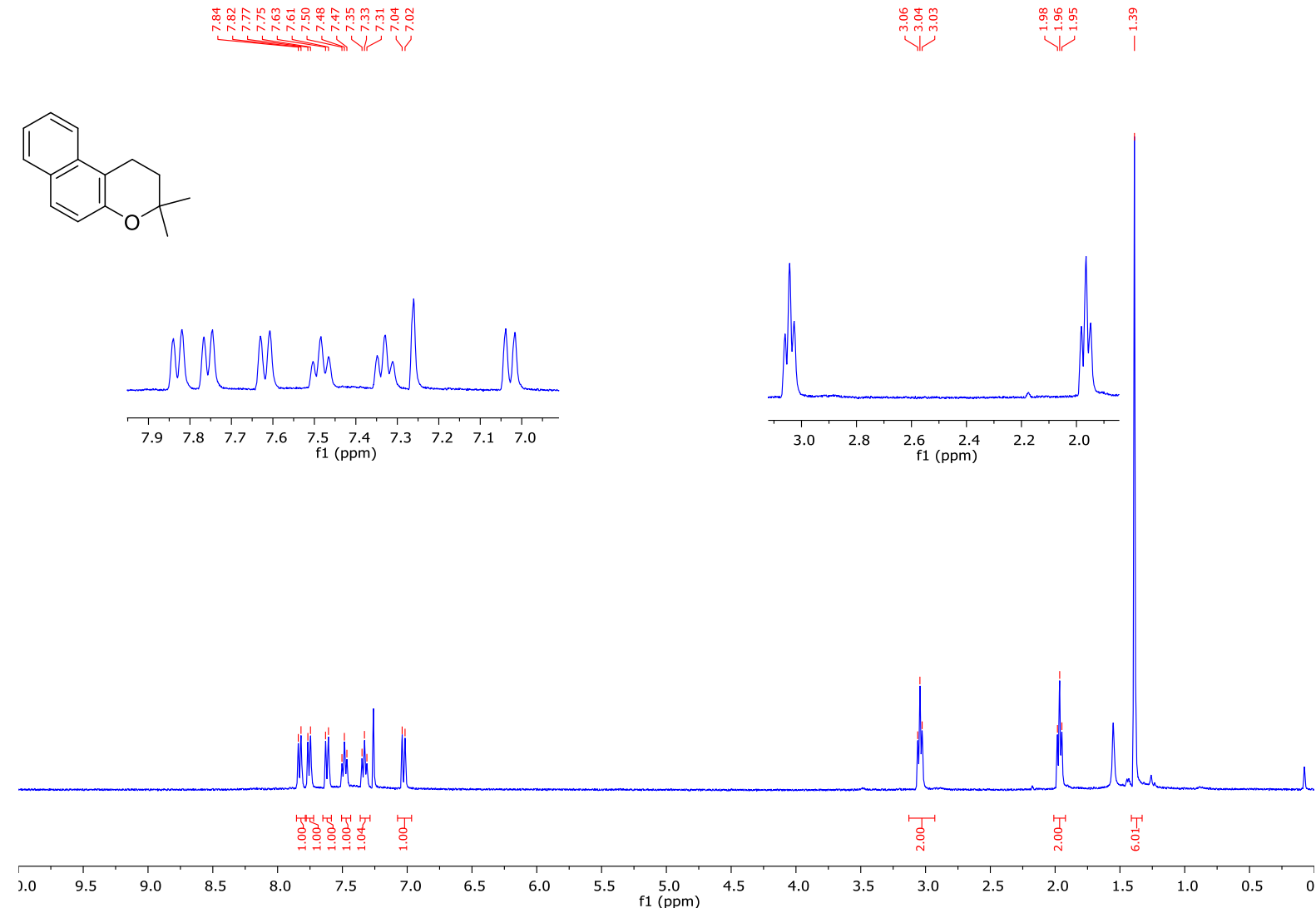

${ }^{13} \mathrm{C}$ NMR (101 MHz, $\left.\mathrm{CDCl}_{3}\right)$

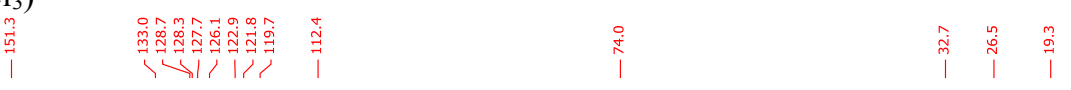

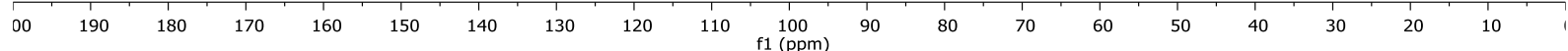


3u,${ }^{1} \mathrm{H}$ NMR $\left(400 \mathrm{MHz}, \mathrm{CDCl}_{3}\right)$

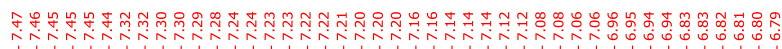

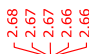<smiles>c1ccc(C2(c3ccccc3)Cc3ccccc3O2)cc1</smiles>
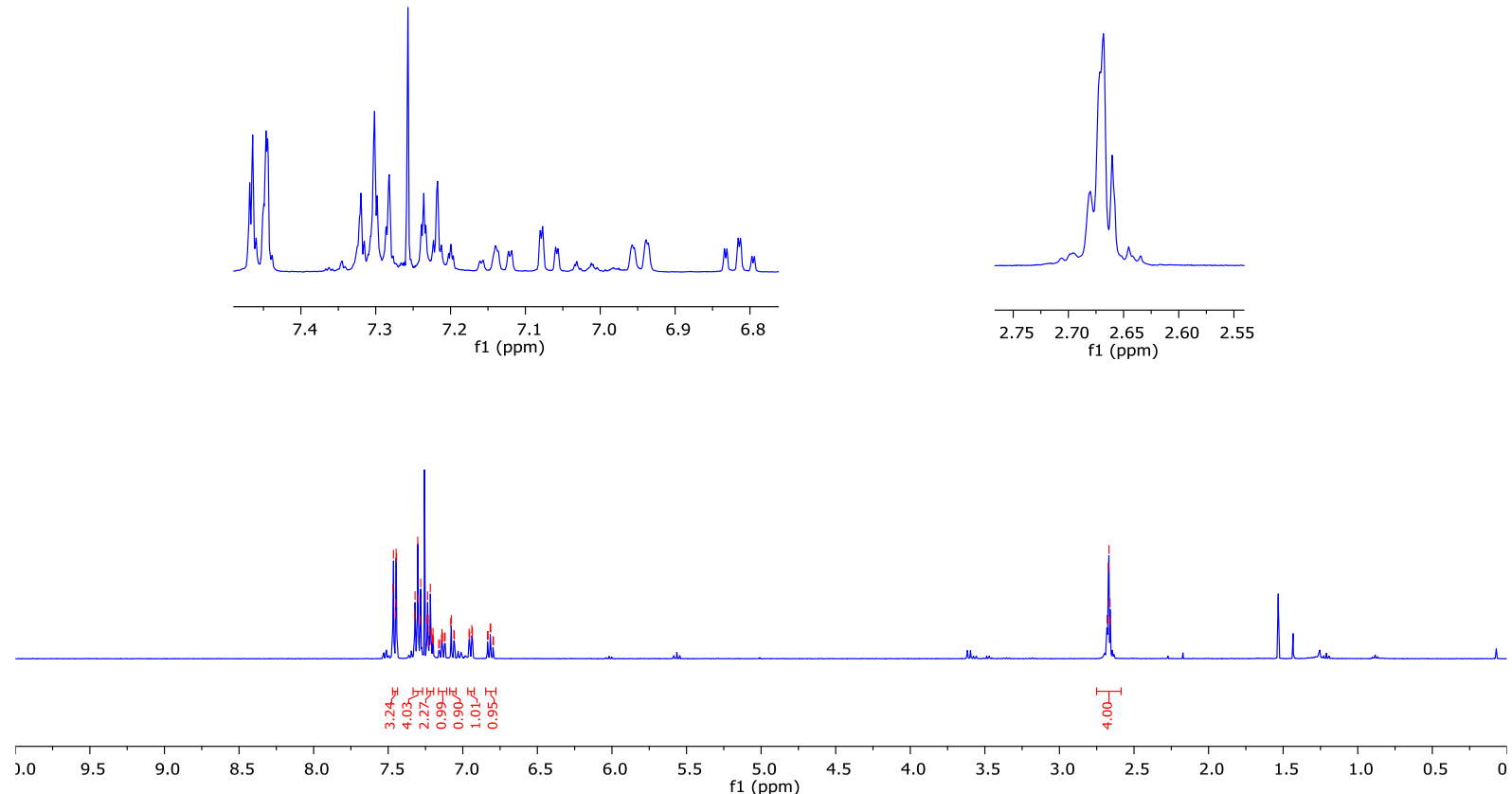

${ }^{13} \mathrm{C}$ NMR (101 MHz, $\left.\mathrm{CDCl}_{3}\right)$

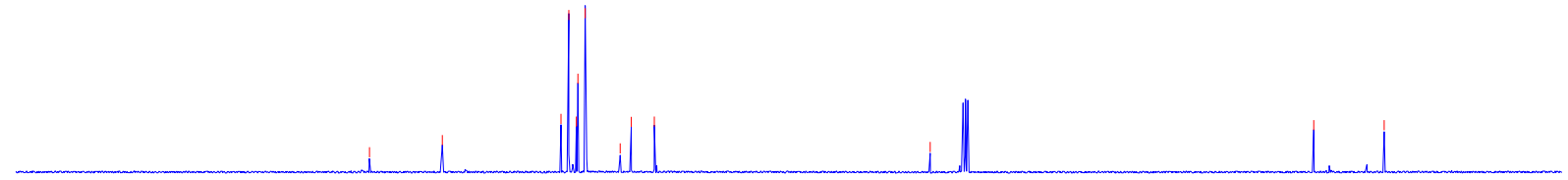

\begin{tabular}{|c|c|}
\hline 170 & 180 \\
\hline
\end{tabular}

